\title{
Neutron Resonance
}

Transmission Analysis

(NRTA): A Nondestructive

Assay Technique for the Next

Generation Safeguards

Initiative's Plutonium Assay

Challenge

J. W. Sterbentz

D. L. Chichester

December 2010

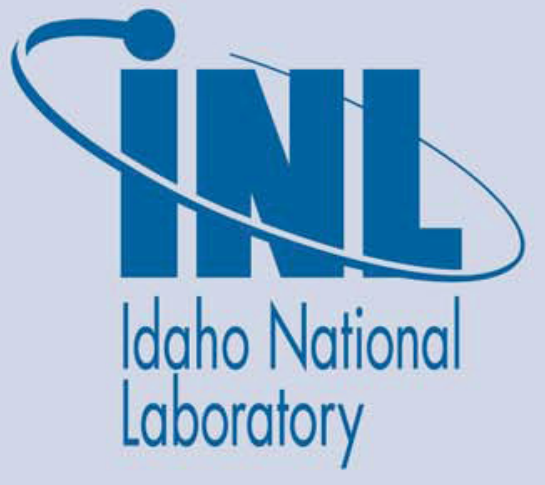

INL is a U.S. Department of Energy National Laboratory operated by Battelle Energy Alliance 


\section{DISCLAIMER}

This information was prepared as an account of work sponsored by an agency of the U.S. Government. Neither the U.S. Government nor any agency thereof, nor any of their employees, makes any warranty, expressed or implied, or assumes any legal liability or responsibility for the accuracy, completeness, or usefulness, of any information, apparatus, product, or process disclosed, or represents that its use would not infringe privately owned rights. References herein to any specific commercial product, process, or service by trade name, trade mark, manufacturer, or otherwise, do not necessarily constitute or imply its endorsement, recommendation, or favoring by the U.S. Government or any agency thereof. The views and opinions of authors expressed herein do not necessarily state or reflect those of the U.S. Government or any agency thereof. 
INL/EXT-10-20620

\title{
Neutron Resonance Transmission Analysis (NRTA): A Nondestructive Assay Technique for the Next Generation Safeguards Initiative's Plutonium Assay Challenge
}

\author{
J. W. Sterbentz \\ D. L. Chichester
}

December 2010

Idaho National Laboratory
Idaho Falls, Idaho 83415

http://www.inl.gov 


\section{ACKNOWLEDGEMENT}

The authors would like to acknowledge and thank Dr. Steve Tobin of Los Alamos National Laboratory for his assistance in coordinating and explaining the overarching goals of the NGSI spent fuel assay project and in allowing us access to the LANL resources developed to support spent-fuel assay research and development. The work in this report was sponsored by the National Nuclear Security Administration Office of Nonproliferation and International Security (NA-241). 


\section{EXECUTIVE SUMMARY}

This is an end-of-year report for a project funded by the National Nuclear Security Administration's Office of Nuclear Safeguards (NA-241). The goal of this project is to investigate the feasibility of using Neutron Resonance Transmission Analysis (NRTA) to assay plutonium in commercial light-water-reactor spent fuel. This project is part of a larger research effort within the Next-Generation Safeguards Initiative (NGSI) to evaluate methods for assaying plutonium in spent fuel, the Plutonium Assay Challenge. The first-year goals for this project were modest and included: 1) developing a zero-order MCNP model for the NRTA technique, simulating data results presented in the literature, 2) completing a preliminary set of studies investigating important design and performance characteristics for the NRTA measurement technique, and 3) documentation of this work in an end of the year report (this report). Research teams at Los Alamos National Laboratory (LANL), Lawrence Berkeley National Laboratory (LBNL), Pacific Northwest National Laboratory (PNNL), and at several universities are also working to investigate plutonium assay methods for spent-fuel safeguards. While the NRTA technique is well proven in the scientific literature for assaying individual spent fuel pins, it is a newcomer to the current NGSI efforts studying Pu assay method techniques having just started in March 2010; several analytical techniques have been under investigation within this program for two to three years or more. This report summarizes a nine month period of work.

Preliminary results indicate that NRTA has great potential for being able to assay intact spent fuel assemblies. It can identify four plutonium isotopes $\left({ }^{239} \mathrm{Pu},{ }^{240} \mathrm{Pu},{ }^{241} \mathrm{Pu}\right.$, and $\left.{ }^{242} \mathrm{Pu}\right)$, three uranium isotopes $\left({ }^{235} \mathrm{U},{ }^{236} \mathrm{U}\right.$, and $\left.{ }^{238} \mathrm{U}\right)$, and six resonant fission products $\left({ }^{99} \mathrm{Tc},{ }^{103} \mathrm{Rh},{ }^{131} \mathrm{Xe},{ }^{133} \mathrm{Cs},{ }^{145} \mathrm{Nd}\right.$, and $\left.{ }^{152} \mathrm{Sm}\right)$. It can determine the areal density or mass of these isotopes in single- or multiple-pin integral transmission scans. Multiple, difficult-to-hoax observables exist to allow the detection of material diversion (pin defects) including fast-neutron and $\mathrm{x}$-ray radiography, gross transmission neutron counting (uranium-oxygen macroscopic cross-section spoofing), plutonium absorption analysis (plutonium diversion), and fission-product resonance absorption analysis (analysis of fission product ratio agreement with both declared and measured/verified fuel burn-up). Initial benchmark modeling has shown excellent agreement with previously published experimental data for measurements where plutonium assays were experimentally demonstrated to have a precision of better than $3 \%$. Analyses of full $17 \times 17$ pressurized water reactor (PWR) fuel assembly arrays, of varying initial enrichments, cooling times, and burnups, suggest a scale-up of the NRTA technique is possible while still maintaining a measurement precision in the $1 \%$ to $4 \%$ range. The results suggest sufficient transmission strength and signal differentiability is possible for assaying up to 8-pin rows. For an 8-pin maximum assay (looking at diagonal slices of an assembly), 64\% of the fuel pins in an assembly can be directly part of integral transmission assay measurements. Collimated neutron beams or two-dimensional neutron detectors that can spatially resolve a fuel pin diameter have the potential to assay roughly the outer one-third of a pin lineup for up to 12-pin rows. For a 12-pin maximum, $100 \%$ of the fuel pins in an assembly can be part of one or more integral transmission assay measurements. 
The NRTA technique requires an intense pulsed-neutron source in order to achieve sufficient neutron transmission through a fuel assembly in reasonable counting times. One efficient approach for generating such a neutron source is to use a linear electron accelerator (LINAC) coupled with a photoneutron converter and a neutron moderator assembly. Other accelerator types may also be applicable. A conceptual NRTA system design has been presented that will be the basis for future modeling work, trade studies, and performance evaluations in the next year. The design includes the use of an electron accelerator with an endpoint energy in the $10-\mathrm{MeV}$ to $12-\mathrm{MeV}$ range, evacuated drift tubes totaling 4- to 6-meters in length, and collimated neutron detectors such as ${ }^{3} \mathrm{He}$-proportional counters, boron-containing proportional counters, and ${ }^{6} \mathrm{Li}$-doped glass scintillators. 


\section{CONTENTS}

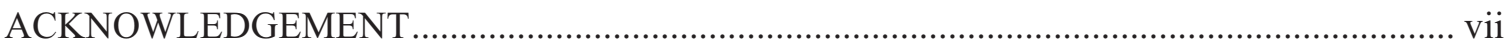

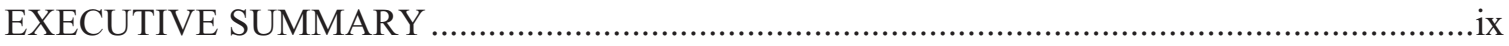

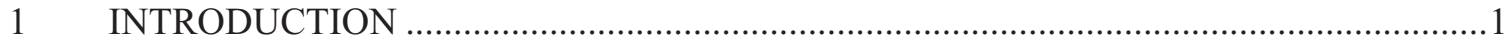

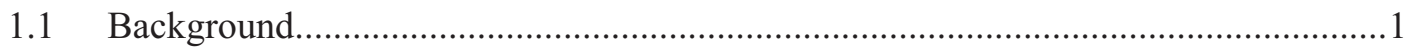

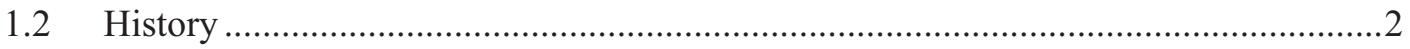

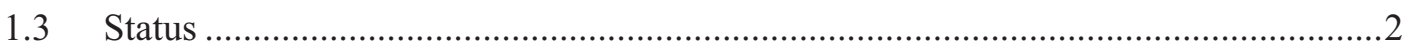

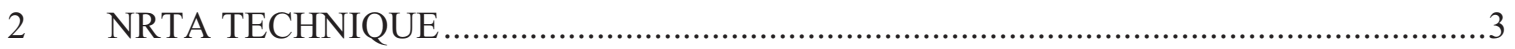

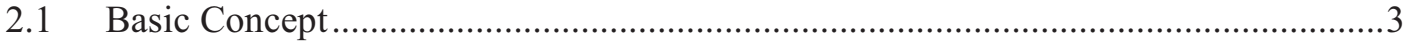

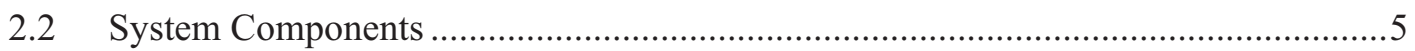

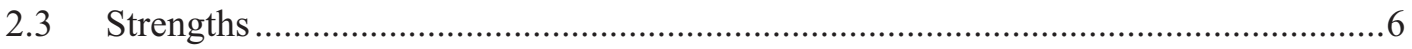

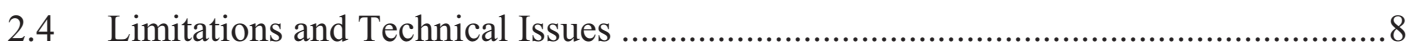

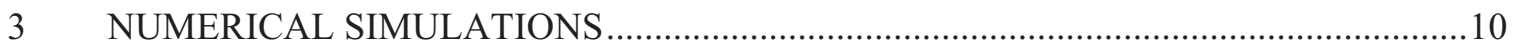

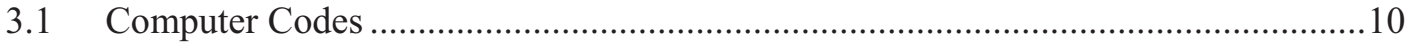

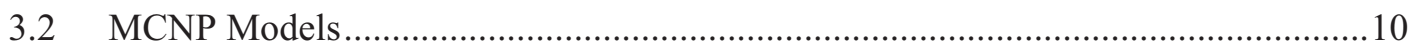

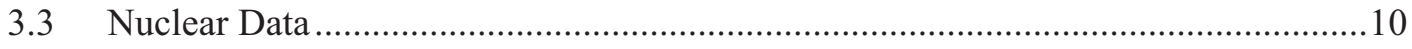

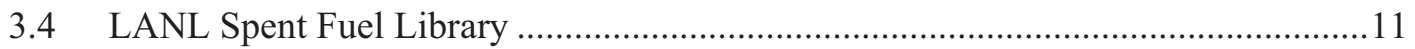

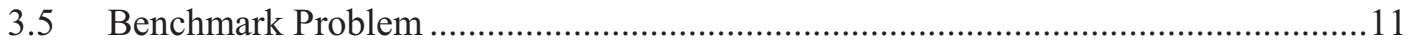

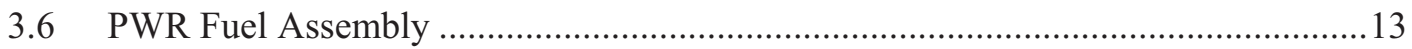

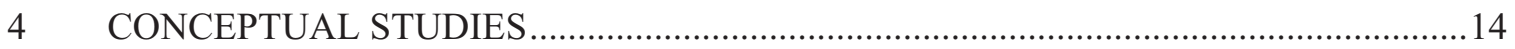

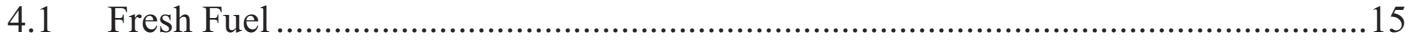

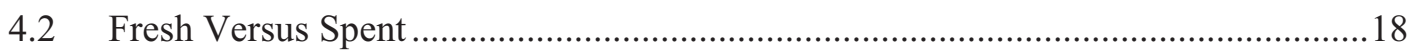

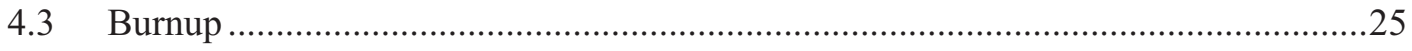

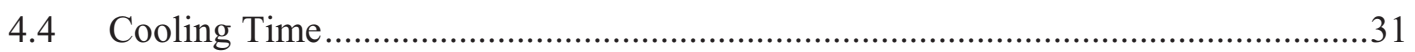

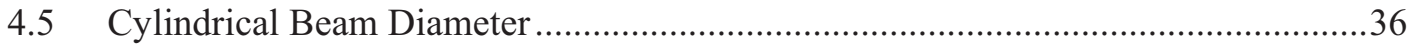

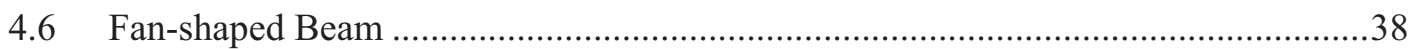

4.7 Directed Point Source - Pencil Beam Irradiation .................................................... 38

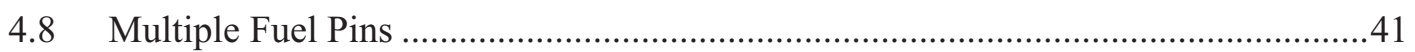

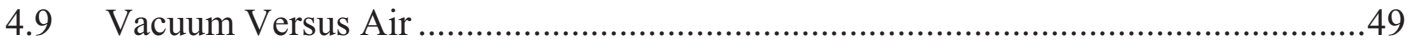

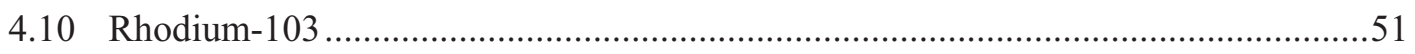

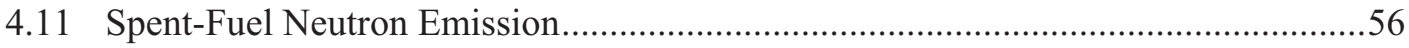

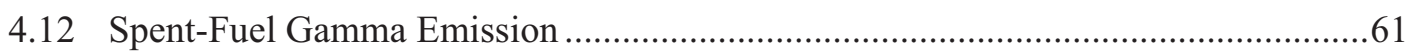

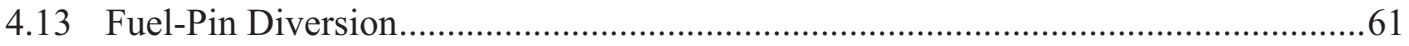

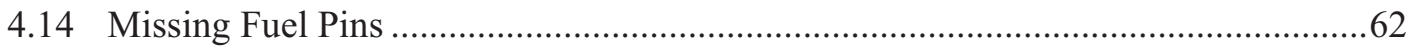

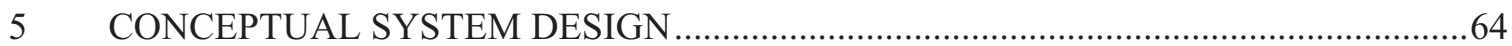




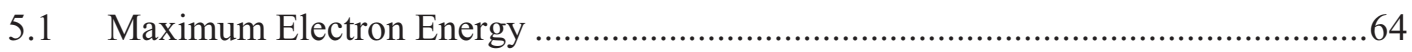

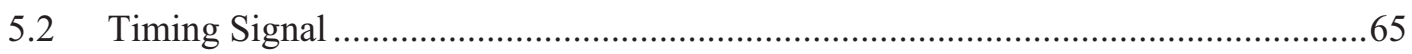

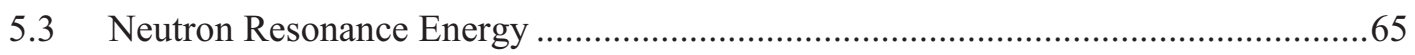

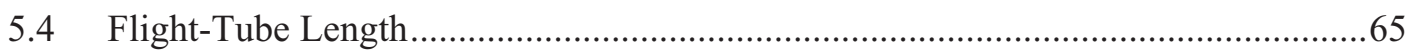

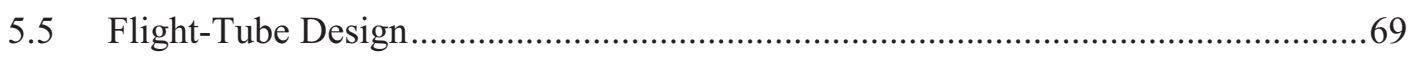

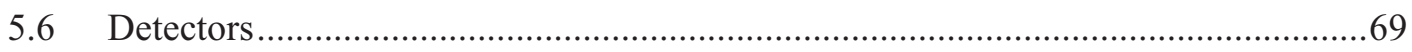

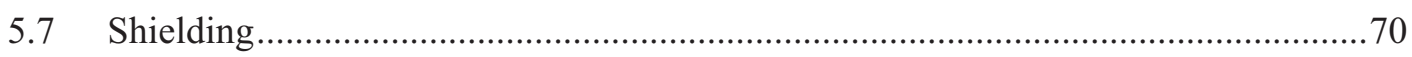

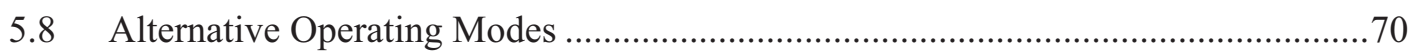

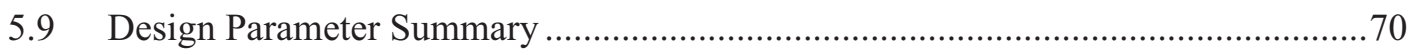

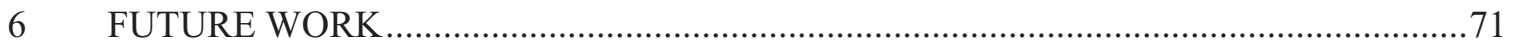

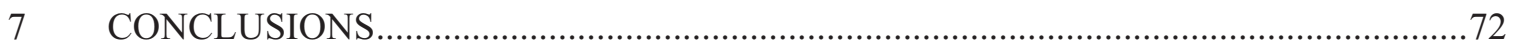

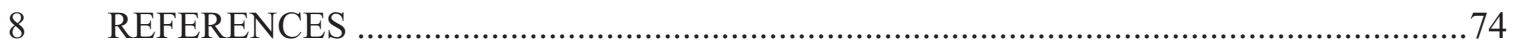

\section{FIGURES}

Figure 1 Basic NRTA concept and component layout.

Figure 2 Measured resonance transmission spectra as a function of neutron energy the spent fuel samples (Spectrum A is for fuel cut from the center of the fuel pin and spectrum $B$ is cut from one end of the fuel pin).

Figure 3 MCNP-calculated transmission spectrum as a function of neutron energy through a single spent fuel pin; important isotopes have been highlighted in red........ 12

Figure 4 PWR $17 \times 17$ fuel assembly showing number of fuel pins in the vertical

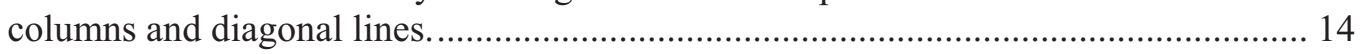

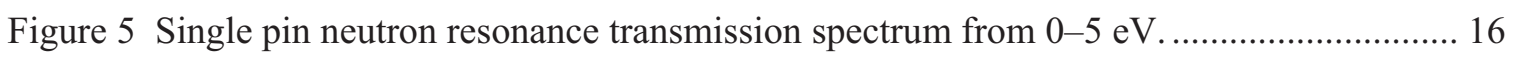

Figure 6 Single pin neutron resonance transmission spectrum from $0-40 \mathrm{eV}$........................... 17

Figure 7 Single pin neutron resonance transmission spectrum from $40-100 \mathrm{eV}$......................... 18

Figure 8 Single pin neutron resonance transmission spectrums for fresh and spent $\mathrm{UO}_{2}$ fuel over the $0-40 \mathrm{eV}$ neutron energy range.

Figure 9 Single pin neutron resonance transmission spectrums for fresh and spent $\mathrm{UO}_{2}$ fuel over the $40-100 \mathrm{eV}$ neutron energy range............................................................ 20

Figure 10 Single pin neutron resonance transmission spectrums for fresh and spent $\mathrm{UO}_{2}$

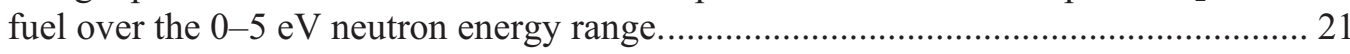

Figure 11 Single pin neutron resonance transmission spectrums for fresh and spent $\mathrm{UO}_{2}$ fuel over the $5-10 \mathrm{eV}$ neutron energy range.

Figure 12 Single pin neutron resonance transmission spectrums for fresh and spent $\mathrm{UO}_{2}$ fuel over the $10-15 \mathrm{eV}$ neutron energy range

Figure 13 Single pin neutron resonance transmission spectrums for fresh and spent $\mathrm{UO}_{2}$ fuel over the 15-20 eV neutron energy range. 
Figure 14 Single pin neutron resonance transmission spectrums for fresh and spent $\mathrm{UO}_{2}$ fuel over the $20-40 \mathrm{eV}$ neutron energy range.

Figure 15 Transmission spectra as a function of burnup over the $0-40 \mathrm{eV}$ energy range. .......... 26

Figure 16 Transmission spectra as a function of burnup over the $0-2 \mathrm{eV}$ energy range. ............. 27

Figure 17 Transmission spectra as a function of burnup over the $0-10 \mathrm{eV}$ energy range. ........... 28

Figure 18 Transmission spectra as a function of burnup over the $10-20 \mathrm{eV}$ energy range. ......... 29

Figure 19 Transmission spectra as a function of burnup over the $20-30 \mathrm{eV}$ energy range. ......... 30

Figure 20 Transmission spectra as a function of burnup over the $30-40 \mathrm{eV}$ energy range. ......... 31

Figure 21 Transmission spectra as a function of cooling time for a fixed $30 \mathrm{GWD} / \mathrm{MTU}$

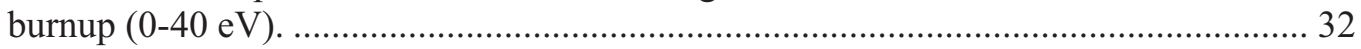

Figure 22 Transmission spectra as a function of cooling time for a fixed $30 \mathrm{GWD} / \mathrm{MTU}$

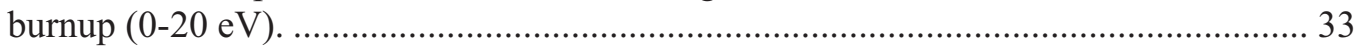

Figure 23 Transmission spectra as a function of cooling time for a fixed $30 \mathrm{GWD} / \mathrm{MTU}$

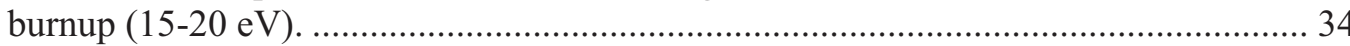

Figure 24 Transmission spectra as a function of cooling time for a fixed 60 GWD/MTU

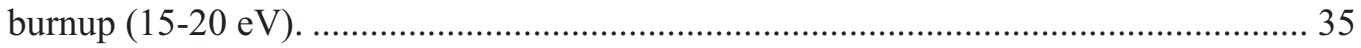

Figure 25 MCNP-calculated transmitted signal as a function of cylindrical beam diameter.

Figure 26 Geometry for the directed point source and fuel pin.

Figure 27 Neutron transmission spectra for a directed point source across a $\mathrm{UO}_{2}$ pellet/pin over the $0-40 \mathrm{eV}$ energy range.

Figure 28 Neutron transmission spectra for a directed point source across a $\mathrm{UO}_{2}$ pellet/pin over just the $18-20 \mathrm{eV}$ energy range.

Figure 29 Neutron resonance transmission spectra through multiple spent fuel pins (0-40 $\mathrm{eV})$ at $\mathrm{y}=0.0 \mathrm{~cm}$.

Figure 30 Neutron resonance transmission spectra through multiple spent fuel pins (0-5 $\mathrm{eV})$ at $\mathrm{y}=0.0 \mathrm{~cm}$.

Figure 31 Neutron resonance transmission spectra through multiple spent fuel pins (0-20 $\mathrm{eV}$ ) at $\mathrm{y}=0.0 \mathrm{~cm}$.

Figure 32 Neutron resonance transmission spectra through multiple spent fuel pins (20$40 \mathrm{eV}$ ) at $\mathrm{y}=0.0 \mathrm{~cm}$.

Figure 33 Neutron resonance transmission spectra through multiple spent fuel pins (0-40 $\mathrm{eV}$ ) at $\mathrm{y}=0.375 \mathrm{~cm}$.

Figure 34 Neutron resonance transmission spectra through multiple spent fuel pins (0-5 $\mathrm{eV}$ ) at $\mathrm{y}=0.375 \mathrm{~cm}$.

Figure 35 Neutron resonance transmission spectra through multiple spent fuel pins (0-20 $\mathrm{eV}$ ) at $\mathrm{y}=0.375 \mathrm{~cm}$.

Figure 36 Neutron resonance transmission spectra through multiple spent fuel pins (20$40 \mathrm{eV})$ at $\mathrm{y}=0.375 \mathrm{~cm}$.

Figure 37 Neutron transmission through different flight tube lengths filled with air 50 
Figure 38 Transmission spectra for $15 \mathrm{GWD} / \mathrm{MTU}$ burnup with and without ${ }^{103} \mathrm{Rh}$ over the $0-40 \mathrm{eV}$ energy range

Figure 39 Transmission spectra for $15 \mathrm{GWD} / \mathrm{MTU}$ burnup with and without ${ }^{103} \mathrm{Rh}$ over the $0-2$ eV energy range.

Figure 40 Transmission spectra for $30 \mathrm{GWD} / \mathrm{MTU}$ burnup with and without ${ }^{103} \mathrm{Rh}$ over the $0-2 \mathrm{eV}$ energy range

Figure 41 Transmission spectra for $45 \mathrm{GWD} / \mathrm{MTU}$ burnup with and without ${ }^{103} \mathrm{Rh}$ over the $0-2 \mathrm{eV}$ energy range.

Figure 42 Total neutron emission rate versus cooling time for a single PWR spent fuel assembly (15 GWD/MTU).

Figure 43 Total neutron emission rate versus cooling time for a single PWR spent fuel assembly (30 GWD/MTU).

Figure 44 Total neutron emission rate versus cooling time for a single PWR spent fuel assembly (45 GWD/MTU).

Figure 45 Total neutron emission rate versus cooling time for a single PWR spent fuel assembly (60 GWD/MTU).

Figure 46 Total neutron emission rate for a single PWR spent fuel assembly as a function of burnup

Figure 47 Total integrated transmission flux for mono-energetic neutron beams incident on rows of PWR $\mathrm{UO}_{2}$ spent fuel pins.

\section{TABLES}

Table 1 Spent fuel assembly neutron source strength or emission rate (neutrons/second)......... 56

Table 2 Calculated k-effectives and multiplication factors for a single assembly in vacuum.

Table 3 Total cross section and mean-free-path (mfp) for $\mathrm{UO}_{2}$ fuel.

Table 4 Neutron flight times for different flight tube lengths and neutron energies.

Table 5 Accelerator pulse rate and corresponding cycle length.

Table 6 Initial NRTA System Parameters 



\section{Neutron Resonance Transmission Analysis (NRTA): A Nondestructive Assay Technique for the Next Generation Safeguards Initiative's Plutonium Assay Challenge}

\section{INTRODUCTION}

This report documents work performed by Idaho National Laboratory over a ninemonth period in 2010 to evaluate the non-destructive assay technique known as neutronresonance transmission analysis (NRTA) as a method for quantifying plutonium in spent nuclear fuel. This project has been sponsored by the National Nuclear Security Administration's Next Generation Safeguards Initiative and is a part of a larger set of investigations aimed at identifying and studying nondestructive methods for assaying spent fuel.[1] The first phase of the project has been focused primarily on conceptual studies to assess if the NRTA technique is a viable means to assay spent fuel, first determining if the transmission of thermal neutrons through a representative spent fuel assembly is sufficient to assay more than one fuel pin and then quantifying this behavior.

\subsection{Background}

New analytical methods are needed to assay spent nuclear fuel and determine fissile material inventories. Ideally, these measurements will be able to assay whole, unaltered commercial-nuclear-reactor assemblies without the need for disassembly, sectioning, or chopping, providing "head-end" inventory data for materials entering current-generation and future spent-fuel reprocessing/recycling facilities. Quantitative measurements of plutonium entries into safeguarded fuel reprocessing facilities prior to material decomposition will provide a higher level of confidence for the actual plutonium mass than found with the current approach, which relies on vendor-supplied burn-up calculations. One potential method to accomplish this task is neutron resonance transmission analysis, NRTA.

The NRTA concept is based on solid theoretical principles, has been demonstrated experimentally at the bench scale using commercial spent fuel, and has achieved a plutonium assay measurement precision of $2-4 \%$ in ad-hoc testing. [2-4] The technique uses a pulsed accelerator to produce an intense, short pulse of neutrons. These neutrons, traveling at different speeds according to their energy in a time-of-flight (TOF) configuration, are used to interrogate a spent fuel assembly. Neutron transmission through the assembly is monitored as a function of neutron energy (time after the pulse), similar to the way neutron cross-section data is often collected. Neutron detection is performed using a high-rate sensor, such as a lithiated-glass scintillation detector.[3-5] Results are read from the detector as count rate versus time, with faster (higher-energy) neutrons arriving first and slower (lower-energy) neutrons arriving later. The low-energy elastic scattering and absorption resonances of plutonium and other isotopes modulate the transmitted neutron spectrum. The plutonium content in the fuel can be determined by analyzing this attenuation. The data format is similar to that from a Lead Slowing-Down 
Spectrometer (LSDS).[6] However, unlike in LSDS measurements the fuel is does not play an important role in the neutron thermalization process. Unknown material impurities from hydrides (hydrogen diffusion into cladding metals) or extreme fission product loadings (which may be present in advanced high-burnup mixed-oxide or minoractinide bearing fuels) in the spent fuel will not detrimentally alter the neutron sourceterm energy distribution in a way that impacts the NRTA measurement.

\subsection{History}

The NRTA concept is not new. The first paper (reference 7) on the subject was published in 1975, followed by substantial research through the mid-1980s. Most of the published research literature is from the National Bureau of Standards (NBS); many of these NBS papers are cited in this report. Much of the published literature is focused on applications related to neutron radiography, in particular, the measurement of uranium in waste materials and the assay of isotopic heterogeneities in welds, brazes, and metals.[813] One NRTA study looked at optimizing the accelerator neutron output.[14] Before NRTA research and publishing literature diminished, around 1984, several papers documented the use of NRTA to characterize a PWR spent fuel pellet, a pellet with approximately 25 GWD/MTU burnup.[2-5] These four NRTA spent-fuel papers have provided confirmation that the NRTA concept is a viable and potentially very powerful spent assay technique.

These same four NRTA spent-fuel papers provide important benchmark data that is used here to provide a level of validation with MCNP-calculated transmission spectra, providing justification for the use of the MCNP computer code, models, cross-section data, and calculated results in this report. The MCNP code and models have been used to assess the important question of what is the maximum number of PWR fuel pins the NRTA technique can penetrate and assay. Plus, the calculations have been extremely useful in addressing other issues, including: (1) identifying which resonance depressions corresponded to which spent-fuel isotopes, (2) understanding the effects of assembly burnup and cooling time on NRTA measurements, (3) establishing the impact of spentfuel assembly neutron emission on the NRTA signal background and how to minimize this effect, and (4) limitations of the NRTA technique. The conceptual study calculations have also provided a basis for developing a preliminary NRTA system design with emphasis on the minimization of the size of the NRTA physical footprint.

\subsection{Status}

In March 2010, Idaho National Laboratory (INL) started a project to evaluate the feasibility of using NRTA to address the spent fuel plutonium assay challenge of the Next Generation Safeguards Initiative (NGSI). This evaluation joined a much larger research program evaluating over a dozen other potential measurement techniques already in progress. The first-year deliverables of the NRTA project were to (1) develop a zeroorder MCNP model for the NRTA technique, (2) complete a preliminary study of the NRTA technique for assaying PWR spent-fuel assemblies, (3) perform a literature search, and (4) document first-year activities in an end of year report. Despite the late start, the INL NRTA project has made good progress in the preliminary assessment and the calculated results look promising. 
The conceptual or feasibility studies have shown sufficient neutron penetration into the assembly to quantitatively assay up to at least 8, and possibly 12 , PWR-type fuel pins and qualitatively detect plutonium mass diversion from any pin within a PWR fuel assembly. Limited by fundamental physics, this level of sensitivity into the inner region of a PWR fuel assembly is comparable to other techniques, and exceeds most.

The next phase of the NRTA project (fiscal year 2011) will include additional feasibility studies, evaluation of the three PWR diversion scenarios, and correction of the deuterium photonuclear cross-section data for use in MCNPX. Work will then focus on accelerator, target, and filter design with the goal to optimize and maximize the neutron output plus studies to evaluate neutron detector design as an integrated system component and a preliminary evaluation of the analytical tools needed to evaluate NRTA measured transmission spectra. Other tasks, as time and funds permit, will include the study of alternative modes of NRTA system operation and assay, NRTA as a mobile system, and analysis of hypothetical transmission spectra.

\section{NRTA TECHNIQUE} physics.

This section provides a basic discussion of the NRTA concept and underlying

\subsection{Basic Concept}

The NRTA technique uses low-energy neutrons as the probing radiation. The low-energy neutrons are thermal and epithermal neutrons in the $0.1-40 \mathrm{eV}$ energy range. This neutron energy range is at the bottom end of the actinide-resonance range, where most actinides have at least one or more resonances. For the spent fuel actinides, as will be seen, the resonances are typically large in magnitude, narrow in breadth, and fortuitously well-separated resulting in distinctive resonance transmission spectra.

Of the hundreds of fission-product isotopes found in spent fuel, only a half-dozen or so have any resonance structure in the 0.1-40 eV energy range. Each of these fission products has only one resonance in this energy range, none interfere with the actinide resonances. All the other isotopes present in the spent fuel - for example, oxygen in the $\mathrm{UO}_{2}$ and zirconium, tin, iron, chromium, oxygen, niobium, nickel, carbon, and silicon in the Zircaloy-4 clad - also have no resonance structure in the 0.1-40 eV range and, therefore, also do not interfere with the resonance transmission signal. The same is true for the hydrogen impurity (hydration) in the clad and air isotopes.

The low-energy neutrons used for NRTA measurements are generated in a complex multi-step process that starts with a pulsed high-energy particle accelerator, such as a LINAC. In the electron accelerator approach, high-energy electrons impact a high-Z converter (e.g., tungsten or tantalum) and produce a continuum of bremsstrahlung photons. It is practical to consider using an accelerator having an endpoint energy in the range of $12 \mathrm{MeV}$ for this discussion. The high-energy bremsstrahlung photons (2-12 $\mathrm{MeV}$ ) then pass through a low-Z photo-neutron converter (heavy water or beryllium) to produce high-energy neutrons. These high-energy neutrons pass through a low-Z neutron moderator (polyethylene, light water, or heavy water) where they scatter and lose energy, a process called thermalization. This scattering process yields a continuous distribution of neutrons, varying in energy from the starting energy down to approximately $0.025 \mathrm{eV}$, 
the thermal kinetic energy of nuclei. It is the portion of the continuum having thermal and epithermal neutrons from 0.1 to $40 \mathrm{eV}$ that is of interest to the NRTA technique.

The thermalized neutrons can then be collimated to create a neutron beam that is directed at a spent fuel assembly. A fraction of the neutrons incident on the fuel assembly scatter out of the beam as they interact with individual fuels pins in the assembly through low-energy elastic scattering, neutron-capture absorption, and neutroncapture fission. The rest of the neutrons pass through the spent fuel assembly unaffected as the transmitted signal. It is these transmitted neutrons - the modulated beam - that is measured in the NRTA technique. The modulated beam, or transmitted signal, is an integral scan of rows of pins in the assembly and is analogous to how a traditional x-ray radiograph is used to detect the presence of dense objects in the human body (bones) and the location of low density regions (bone cracks). The NRTA technique does not assay individual pins with the exception of the four corner pins.

Many advances have been made between the early 1980s and today. These advances help enable practical modeling and, potentially, the implementation of the NRTA technique. The most significant enabling advances have been improvements in the nuclear data needed to analyze NRTA data; neutron cross sections and resonance parameters have been remeasured and evaluated and are now known with a high degree of accuracy. The NRTA technique grew out of cross-section measurement techniques; it is basically the inverse to the problem of measuring neutron cross sections. In the case of neutron cross-section measurements, the two known variables are (1) the sample isotopic content, or sample areal density $N_{i}$ and (2) the transmission flux $T_{i}$ through isotope $i$ from the TOF measurement. The third variable, the total cross section $\sigma_{t i}$ for a isotope $i$, is the unknown variable and the desired quantity to be measured. The total cross section is then calculable as a function of neutron energy, $E$, from Eq. 1 .

$$
\mathrm{T}_{\mathrm{i}}(\mathrm{E})=\mathrm{e}^{-\mathrm{N}_{\mathrm{i}} \sigma_{\mathrm{ti}}(\mathrm{E})}
$$

For NRTA, the unknown variable is the isotopic areal density $N_{i}$, and the two known variables are the total cross section $\sigma_{t i}$ and the measured neutron transmission flux $T_{i}$. The same formula above is then used to calculate the areal density. Other known factors include the fuel pin and assembly geometry and dimensions, cladding material, and the fuel form $\left(\mathrm{UO}_{2}\right)$. Knowledge of these "other" factors should significantly reduce the systematic error, improve the overall NRTA measurement accuracy, and promote the use of calibration standards.

Calibration standards will play an important role in the assessment of the first NRTA system, but also in all subsequent spent fuel measurements to calibrate the system, establish biases, and reduce systematic error. Combinations of pins and assemblies with known dimensions and material compositions can be used as calibration standards. For example, pins containing (1) no fuel, (2) fresh $\mathrm{UO}_{2}$ with $100 \%{ }^{238} \mathrm{U}$ and various ${ }^{235} \mathrm{U}$ enrichments, (3) and spent fuel surrogate compositions (uranium, plutonium, and fission product isotopes) can all be fabricated with accurately known isotopic compositions.

High-fidelity burnup calculations can also play an important role and further support NRTA spent-fuel transmission measurements. NRTA transmission measurements can be compared to calculated transmission measurements. Agreement 
between measured and calculated spectra, and then comparison of the corresponding isotopic concentrations, can be used to confirm pin/assembly isotopic concentrations, burnup, and cooling times.

\subsection{System Components}

The major components of an NRTA system include: (1) a linear electron accelerator capable of pulsed operation, (2) converter, photoneutron targets, filters, collimators, and shielding, (3) time-of-flight drift tube, (4) vertical chamber to contain and cool a spent fuel assembly, (5) mechanism to lift, rotate, and transversely move the assembly, and (6) neutron detectors with associated TOF electronics for data acquisition (such as a multichannel scalar (MCS) configuration). Figure 1 shows a cartoon of the basic NRTA concept and component layout with preliminary material and accelerator parameters.

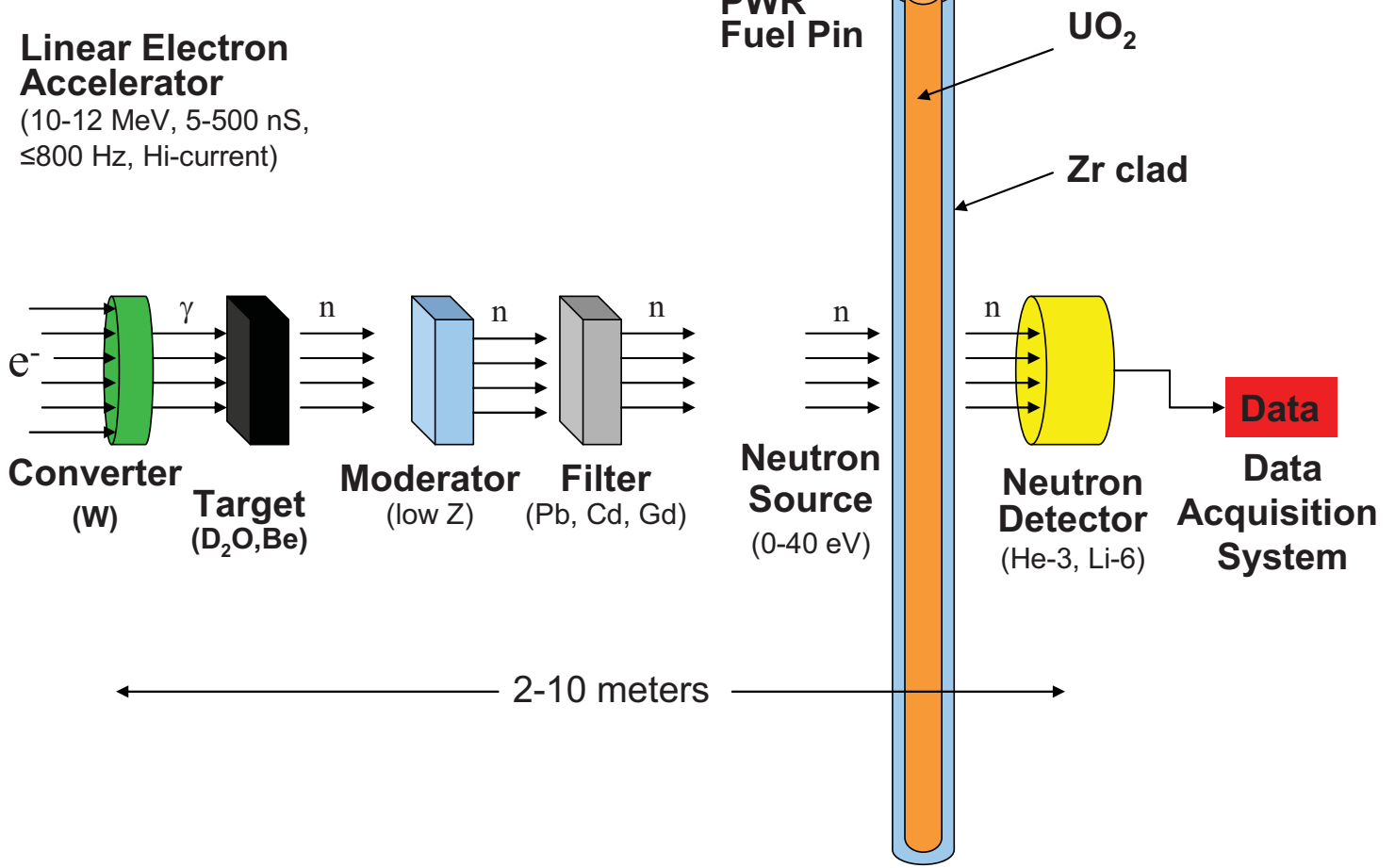

Figure 1 Basic NRTA concept and component layout.

The neutron time-of-flight (TOF) method, with a precisely known flight-tube length, will be used to measure the transmission neutron energies. Neutron detection is performed using a high-rate sensor, such as a helium-3 proportional counter or lithiatedglass scintillation detector. Results are read from the detector as counts versus time, with faster neutrons (higher-energy neutrons) arriving first and slower neutrons (lower-energy neutrons) arriving later.

The low-energy fission, capture, and elastic scattering resonances of plutonium and other isotopes modulate the transmitted-neutron spectrum. Spectrum modulation, or neutron attenuation, appears as depressions in the collected data that correspond to energy-specific resonances and, hence, specific and identifiable isotopes. The plutonium 
content in the fuel can be determined by analyzing the appropriate depression signals (magnitude or area of the depression).

The NRTA technique will require transmission measurements with and without the spent fuel assembly. A transmission measurement without the assembly is needed to provide a baseline measurement, or the unperturbed transmission flux spectrum, from which the actinide-resonance flux depressions are measured. A transmission measurement of rows with multiple pins can result in significant out-scatter of neutrons from the transmission signal. Calibration standards could be used to measure the individual loss effects due to the clad, oxide, and ${ }^{238} \mathrm{U}$. The bulk of the signal loss is due to ${ }^{238} \mathrm{U}$ and the oxide. Adding these signal components to the measured signal would allow the reconstruction of a transmission signal due to the lower concentration actinides and fission products alone, which may improve measurement accuracy.

A PWR assembly assay will require many transmission measurements for a complete assay. In order to accommodate multiple integral assay measurements, the PWR fuel assembly will need to be rotated about its central axis and moved transversely across the neutron interrogating beam. Rotation and transverse movement will allow assay of both the straight and diagonal rows of pins (Figure 4). A full assembly assay would require four 90-degree rotations. Many of the fuel pins will be assayed multiple times during the process as part of different integral row transmission measurements. Due to assembly geometry and burnup symmetry, quadrant assays should be similar, and any deviations between quadrant assays could be treated as a potential diversion.

The NRTA technique is envisioned to assay rows of in-line pins and to produce an integral value for the uranium, plutonium, and fission-product concentrations. Exceptions are the four corner pins in the fuel assembly. The NRTA technique will be able to analyze these four pins individually and to determine the uranium, plutonium, and fission-product concentrations most accurately. These corner-pin measurements could be compared to calculated values from high-fidelity burnup calculations and thereby allow for verification of an assembly burnup and cooling time. Subsequent NRTA measurements of the assembly's multiple pin rows (2-12 pins per row) should then also be agreement with the burnup predictions.

\subsection{Strengths}

As a measurement technique for performing high-precision plutonium assays the NRTA technique has a number of strengths in comparison with other possible nondestructive assay approaches.

1. NRTA has the potential for accurate assay measurements with a precision in the range of $1 \%-4 \%$ uncertainty (this will be the primary focus of our research efforts for fiscal year 2011).

2. NRTA produces distinctive resonance-transmission spectra that can uniquely identify specific actinide and fission product isotopes. The method detects and measures plutonium isotopes directly, it does not rely on correlations or the effective ${ }^{240} \mathrm{Pu}$ concept. 
3. In addition to ${ }^{239} \mathrm{Pu}$, NRTA can identify and assay several additional important fissionable isotopes and spent-fuel actinides directly, including ${ }^{235} \mathrm{U},{ }^{236} \mathrm{U},{ }^{238} \mathrm{U},{ }^{240} \mathrm{Pu}$, ${ }^{241} \mathrm{Pu}$, and ${ }^{242} \mathrm{Pu}$.

4. NRTA can identify the presence of ${ }^{234} \mathrm{U},{ }^{241} \mathrm{Am}$, and ${ }^{243} \mathrm{Am}$. Americium-241 is of particular relevance in higher burn-up fuels (45-60 GWD/MTU) with large cooling times $(>5$ years $)$.

5. NRTA does not suffer resonance interference effects between ${ }^{235} \mathrm{U},{ }^{236} \mathrm{U},{ }^{238} \mathrm{U},{ }^{239} \mathrm{Pu}$, ${ }^{240} \mathrm{Pu},{ }^{241} \mathrm{Pu}$, and ${ }^{242} \mathrm{Pu}$; the resonances are strong, narrow, and well-separated.

6. NRTA can identify 6 resonant fission-product isotopes $\left({ }^{99} \mathrm{Tc},{ }^{103} \mathrm{Rh},{ }^{131} \mathrm{Xe},{ }^{133} \mathrm{Cs}\right.$, ${ }^{145} \mathrm{Nd}$, and ${ }^{152} \mathrm{Sm}$ ) which can potentially be used to estimate assembly burnup, cooling time, and diversion and to verify operator-reported burn-up values.

7. The neutron resonance transmission analysis technique is a mature technology with a solid foundation in theoretical physics.

8. Nuclear data for spent fuel actinide and fission product isotopes are known to high accuracy, especially the total cross section and resonance parameter data needed for assay determinations.

9. NRTA system calibrations with pin/assembly standards can be straightforwardly used to reduce NRTA systematic errors.

10. An NRTA system can be designed to be insensitive to spent-fuel gamma radiation.

11. If a photoneutron source is used as the NRTA radiation source, the accelerator's gamma flash precedes the NRTA measurement without interference.

12. NRTA does not suffer resonance interferences from the presence of oxides in fuel. (Oxides do, however, attenuate the transmitted signal to a small extent.)

13. NRTA does not suffer resonance interferences from zircaloy cladding; only minor signal attenuation occurs due to the cladding because of the relatively small zirconium total-neutron-attenuation cross section.

14. NRTA does not suffer resonance interference from hydrogen impurities in the cladding.

15. The NRTA signal loss from the presence of hydrogen in the cladding is negligible.

16. NRTA does not suffer resonance interferences from the multitude of spent-fuel fission products. The single resonances from each of the six resonant fission products identified so far that are in the energy range of interest for NRTA (i.e., ${ }^{99} \mathrm{Tc},{ }^{103} \mathrm{Rh}$, ${ }^{131} \mathrm{Xe},{ }^{133} \mathrm{Cs},{ }^{145} \mathrm{Nd}$, and ${ }^{152} \mathrm{Sm}$ ) do not interfere with the actinide-isotope resonances.

17. The multiplication factor for a spent fuel assembly in vacuum or air is small; multiplication neutrons are therefore relatively minor and do not significantly affect the transmitted signal for NRTA measurements.

18. Background neutrons from spent fuel (spontaneous fission and alpha-n) should be a minor impact through the judicious use of neutron shielding and since there will be a small solid angle to detectors. Background signal subtraction may also be used. 
19. No isotopic threshold detectors are required; NRTA neutron detectors are total count detectors, and detection can be performed using standard technology.

20. A vacuum is not required in the chamber containing the fuel assembly.

21. Vacuum in the NRTA flight tube may not be required, especially for system designs employing relatively short flight tubes (2-4-meter); in these cases, flight tubes could be air-filled without serious signal attenuation.

22. The NRTA approach may be accurately modeled using conventional neutronics tools, such as MCNP. Relatively simple MCNP models are adequate for NRTA simulations.

23. The NRTA system is suitable for incorporating other NGSI fuel-assay techniques.

\subsection{Limitations and Technical Issues}

Limitations and technical issues associated with the NRTA technique and system are listed below. Three of the issues listed below (1-3) are inherent system requirements or technical limitations due to the nature of the technique. The remaining issues can be addressed with engineering design studies and additional research. For a new facility developed with Safeguards-by-Design concepts, these may not be of significant concern and may eventually become non-issues.

1. The temperature of the spent fuel assembly (spent fuel assembly decay heat) must be measured.

2. The fuel to be assayed may not be immersed in water. The NRTA system could be setup underwater but the fuel assembly and flight tube would still need to be vacuum or air-filled.

3. The range of penetrability of low-energy neutrons into a PWR assembly is fundamentally limited; multiple orthogonal views must be used to reconstruct an assembly's complete burn-up and Pu-inventory profile.

4. An intense thermal/epithermal pulsed neutron source is needed.

5. A large physical infrastructure in needed to accommodate the accelerator, time-offlight tube, detectors, and data analysis system. (However, these requirements will likely be small in comparison with other physical infrastructure requirements needed to allow for the handling of spent fuel.)

6. New data analysis tools are needed.

The NRTA technique may require temperature control equipment and a temperature measurement for the spent fuel assembly. For NRTA, the fuel assembly will necessarily be assayed in air (or vacuum) but not in water. An assembly suspended in vacuum will tend to heat up due to lack of conduction and convection heat-transfer pathways. An assembly suspended in air, however, can be air-cooled through forced convection. In the latter case of forced-air cooling a temperature measurement may not be needed but in vacuum the assembly may heat up, particularly true for assemblies with short cooling times. Heat up of the $\mathrm{UO}_{2}$ fuel that would be expected in an evacuated 
system will Doppler-broaden the resonances; this must be compensated for in the crosssection data that are part of the analytical computer tools.

Low-energy neutron penetrability, or the maximum number of PWR pins that can be assayed in a given row, is limited by the physics of the problem. Beam penetrability, however, is shown in the feasibility studies to be quite good for the NRTA technique.

The NRTA technique will require an intense source of pulsed low-energy neutrons in the range of $10^{12}-10^{13} \mathrm{n} / \mathrm{s}$. The NRTA electron accelerator is expected to be specially designed to maximize the bremsstrahlung photon output and, hence, the neutron output by using a relatively low electron endpoint energy (10-12 MeV). These relatively low energy accelerator electrons will produce a bremsstrahlung photon-energy spectrum that can liberate photo-neutrons from either heavy water $\left({ }^{2} \mathrm{H}\right)$ or beryllium $\left({ }^{9} \mathrm{Be}\right)$ targets; ${ }^{2} \mathrm{H}$ and ${ }^{9} \mathrm{Be}$ have neutron binding energies of 2.2 and $1.67 \mathrm{MeV}$, respectively. The relatively low-energy electrons from the accelerator will also allow the accelerator to maximize the electron current output for a given accelerator power and, thus, maximize the neutron output. In addition, low-energy accelerator electrons will also minimize the thickness of the moderator filter and, in turn, further reduce neutron loss due to outscatter. It is expected that a specially-designed NRTA accelerator could be table-top size, with the capability to vary the maximum electron energy over the 6-15 MeV energy range, similar to a VARITRON accelerator.[15] A charged-particle accelerator may also be suitable for use in an NRTA system, instead of using an electron accelerator. Commercial accelerators using deuteron and proton beams that can generate neutron yields up to $10^{13}$ neutrons per second are currently available.[16] NRTA accelerator design and neutron multiplication optimization studies will be part of the FY2011 tasks.

Accelerator systems used in neutron cross-section measurements are large, highpower systems that typically use $100-\mathrm{MeV}$ or higher electron energies, a $700-800 \mathrm{~Hz}$ repetition rate, and flight-tube lengths up to 200-meters or longer. They operate at powers ranging from 1.5-14.0 kW.[17,18] The optimized NRTA system may operate in the same repetition rate and power range, but at much lower electron energies. The relatively low-energy electrons required when using an electron-accelerator-based neutron source may be of particular value to reduce the size of the accelerator.

The flight tube length is based primarily on the energy range of interest for the transmission neutrons. For the NRTA technique and plutonium assay, neutrons of energy 0.1-20 eV should be sufficient (prior work has used 1-40 eV neutrons). This relatively narrow energy range in the resonance region includes one or more resonances for ${ }^{234} \mathrm{U}$, ${ }^{235} \mathrm{U},{ }^{236} \mathrm{U},{ }^{238} \mathrm{U},{ }^{239} \mathrm{Pu},{ }^{240} \mathrm{Pu},{ }^{241} \mathrm{Pu},{ }^{242} \mathrm{Pu},{ }^{241} \mathrm{Am}$, and ${ }^{243} \mathrm{Am}$ isotope, plus the six resonant fission products ${ }^{145} \mathrm{Nd},{ }^{133} \mathrm{Cs},{ }^{99} \mathrm{Tc},{ }^{152} \mathrm{Sm},{ }^{131} \mathrm{Xe}$, and ${ }^{103} \mathrm{Rh}$. Energies above $20 \mathrm{eV}$, for example 20-40 eV, provides redundant resonances for some of these actinides; hence, there is no need to go to higher energies. Higher energies also require a longer flight tubes for good energy resolution. The NRTA system as proposed here would minimize the flight-tube length and reduce the overall NRTA system footprint from previous work. An NRTA flight-tube length in the range of 2-6 meters is currently envisioned; a basis for the preliminary flight tube lengths is provided in the system design section.

Analytical computer tools will need to be developed to derive isotopic assay estimates from the measured transmission signals. No work has been performed in this area to date. 


\section{NUMERICAL SIMULATIONS}

This section provides information on the numerical computer code simulations used to perform the conceptual studies, also referred to as feasibility studies.

\subsection{Computer Codes}

Both the MCNP5 and MCNPX computer codes were used in the numerical simulations presented here.[19,20] Both codes gave statistically the same results but because the codes resided on different computer systems at INL code usage was based on computer system availability. The models were interchangeable.

\subsection{MCNP Models}

Several MCNP models were developed for the feasibility studies but all were relatively simple in geometry. The simplest geometry models included a directed neutron source, a single fuel pin or line of pins, vacuum flight tube, and flux detector cells. More sophisticated models include a complete PWR $17 \times 17$ pin assembly; the assembly model was taken directly from the LANL 64 library data/models.[21]

The MCNP models used one or more PWR fuel pins in a row. The PWR spentfuel pin geometry was based on the LANL 64 spent fuel library, namely, a $0.82 \mathrm{~cm} \mathrm{UO}_{2}$ pellet diameter, $0.95 \mathrm{~cm}$ diameter clad (no gap), and fuel pin pitch of $1.26 \mathrm{~cm}$. INL MCNP models developed prior to the adoption of the LANL models used similar but slightly different pin-cell dimensions, or a $0.819 \mathrm{~cm} \mathrm{UO}_{2}$ pellet diameter, $0.0082 \mathrm{~cm}$ gap, $0.9498 \mathrm{~cm}$ diameter clad, and pin pitch of $1.25 \mathrm{~cm}$.

In all MCNP models the neutron source was uniformly sampled over the desired transmission neutron-energy range. In an actual physical NRTA system a slowing-down neutron source would be expected to exhibit some non-uniformity, in particular, slightly higher flux at higher energies. In the $0-40 \mathrm{eV}$ energy range, with the use of a low- $\mathrm{Z}$ neutron moderator with low absorption, a relatively flat and uniform energy-flux distribution would be expected. Also, the benchmark data does not exhibit a significant flux tilt to higher energies.

The MCNP models also used two different beam geometries: cylindrical beam and directed point-source beam. The cylindrical beam typically had a diameter less than the $\mathrm{UO}_{2}$ pellet diameter $(<0.82 \mathrm{~cm})$ and was directed through the fuel pin center, such that no neutrons struck the detector flux tally cell without first passing through the $\mathrm{UO}_{2}$ pellet. The cylindrical-beam radius was also varied for some studies. The directed point source is essentially a cylindrical beam with zero radius: a point source directed in a particular direction creating a line of neutrons. The directed point source is useful in order to assess the effect of pellet curvature on the transmission signal.

\subsection{Nuclear Data}

Evaluated Nuclear Data File VII (ENDF-7) was used for most of the numerical simulations.[22] ENDF-5 and ENDF-6 data were used initially and results calculated with these data compare well with the ENDF-7 data. 


\subsection{LANL Spent Fuel Library}

Los Alamos National Laboratory has provided the NGSI working group a comprehensive set of PWR spent-fuel-assembly models, complete with individual spentfuel material compositions for each pin.[23] Three distinct libraries were created for assemblies in air, water, and borated-water. Each of the three libraries included 64 individual spent fuel assemblies, covering the parametric variable space of (1) initial uranium enrichments of 2, 3, 4, and $5 \mathrm{wt}^{2} \%{ }^{235} \mathrm{U}$, (2) burnups of $15,30,45$, and 60 GWD/MTU, and (3) cooling times of 1, 5, 20, and 80 years. The LANL PWR assembly model assumes both quadrant symmetry and symmetry within a quadrant where certain groups of pins have identical burnup. These assumptions allow the 264 total possible fuel pin burnups in the $17 \times 17$ pin array to be reduced to 39 unique pin burnups. Each of the 39 unique burnup pins, however, had four radial burnup zones. Therefore, each of the 64 LANL libraries had a total of $4 \times 39=156$ fuel compositions, using 4 radial material compositions for each of the 39 unique burnup pins in the assembly. Uniform axial burnup was further assumed for each pin radial zone.

The NRTA work here used only the 64 library set for "assembly in air" since the NRTA technique will require the spent fuel assembly to be assayed either in vacuum or air.

\subsection{Benchmark Problem}

Both the MCNP5 and MCNPX codes were used to perform the NRTA numerical simulations for the feasibility studies. In order to develop a level of confidence in the MCNP codes, models, and cross section data, it seemed prudent to compare a calculated transmission spectrum against an actual measured transmission spectrum. Fortunately, two measured spectra were available in the published literature for ad-hoc experiments using a single spent fuel pin.[3]

From the cited reference, the two measured spectra were based on $\mathrm{UO}_{2}$ samples cut from a single spent fuel pin, one from the center of the pin and the other from the end of the pin. Both cylindrical samples were approximately $1.0 \mathrm{~cm}$ in diameter and $2.5 \mathrm{~cm}$ in length (probably a PWR fuel pin). The fuel pin had an estimated burnup of approximately $25 \mathrm{GWD} / \mathrm{MTU}$. No initial enrichment or cooling time was given, but a PWR assembly with approximately 25 GWD/MTU back in the 1970s timeframe was probably around $3.2 \mathrm{wt} \%{ }^{235} \mathrm{U}$.

For comparison purposes, a spent fuel assembly from the LANL 64 library with burnup characteristics closest to the samples was chosen for the transmission calculation. The closest match used a $3.0 \mathrm{wt} \%{ }^{235} \mathrm{U}$ initial enrichment, $30 \mathrm{GWD} / \mathrm{MTU}$ burnup, and a 5-year cooling time. A material composition $(\mathrm{m} 704)$ was arbitrarily chosen from a peripheral pin in the LANL assembly for the MCNP transmission calculation. The burnup characteristics between the actual measured spent-fuel composition and the LANL composition are not exact but are reasonably close.

Figure 2 shows the two measured transmission spectra from the reference paper. Spectrum A, the lower spectrum, is for the sample cut from the center of the fuel pin, and spectrum B, the upper spectrum, is for the sample taken from the end of the fuel pin. Note the ordinate axis discontinuity in this figure. The resonance depressions correspond to specific actinide and fission product isotopes and are clearly marked in the figure. It is interesting to note the deeper depression in spectrum $\mathrm{A}$ for the ${ }^{242} \mathrm{Pu}$ resonance at 2.65 
$\mathrm{eV}$. This is due presumably to the higher burnup at the pin center and correspondingly higher ${ }^{242} \mathrm{Pu}$ concentration. Also, the ${ }^{235} \mathrm{U}$ depression at $8.8 \mathrm{eV}$ is deeper for spectrum $\mathrm{B}$ relative to $\mathrm{A}$ due to the higher ${ }^{235} \mathrm{U}$ concentration remaining at the end of the pin relative to the center.

Figure 3 shows the corresponding MCNP-calculated transmission spectrum. In the LANL 64 library, the spent fuel composition is assumed to be axially uniform, hence the calculated spectrum represents an average over the length of the fuel pin; one might expect the magnitude of the resonance depressions to lie between the two measured spectra. For comparison purposes, the MCNP-calculated spectrum was arbitrarily normalized to the potential scattering levels of spectrum A and B which appear to be approximately equal.

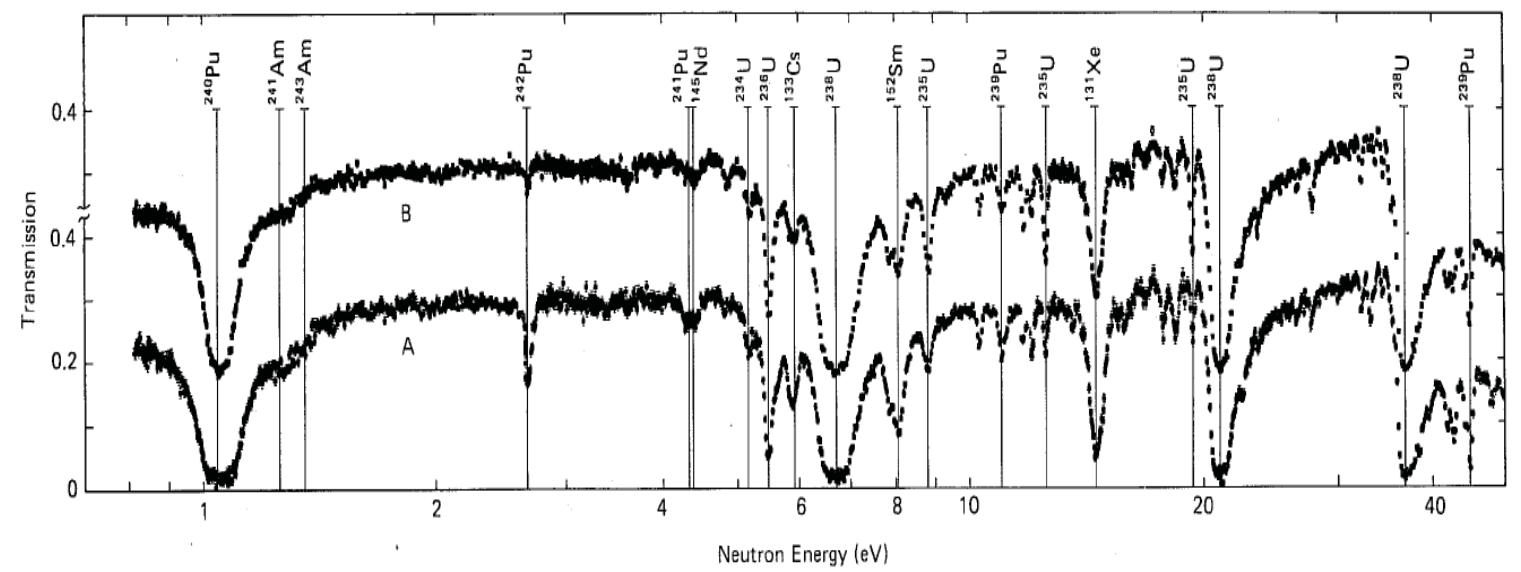

Figure 2 Measured resonance transmission spectra as a function of neutron energy the spent fuel samples (Spectrum $A$ is for fuel cut from the center of the fuel pin and spectrum $B$ is cut from one end of the fuel pin).

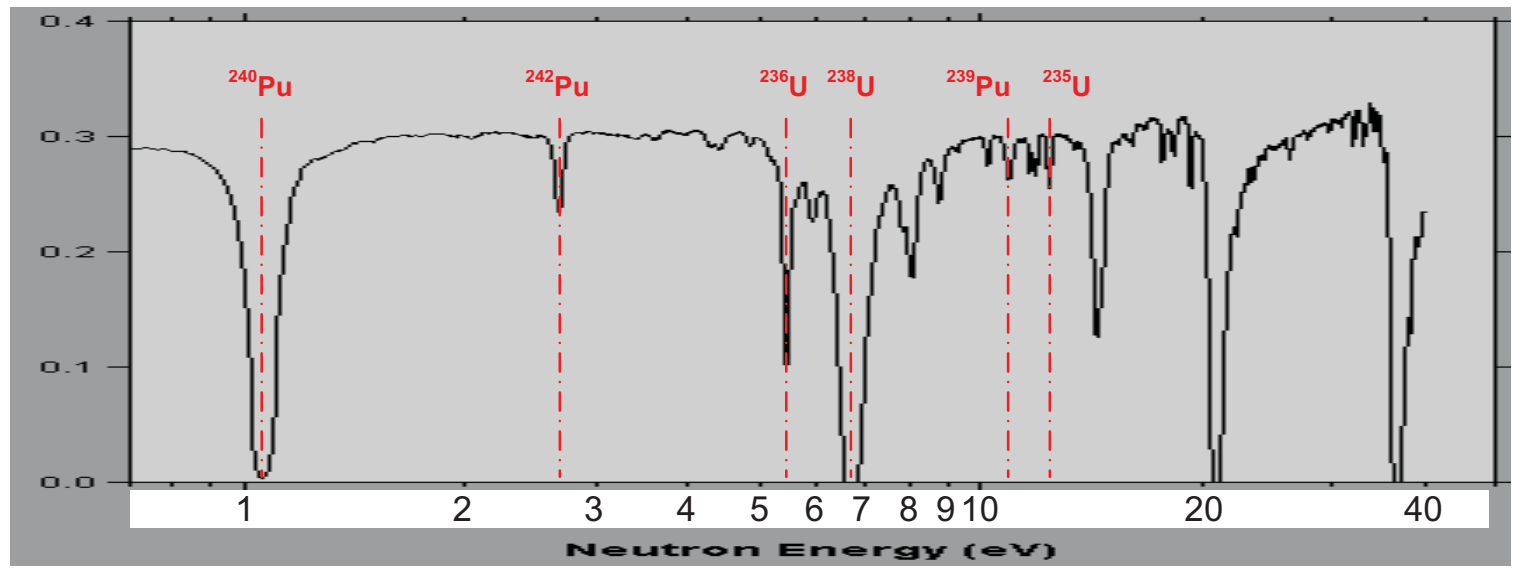

Figure 3 MCNP-calculated transmission spectrum as a function of neutron energy through a single spent fuel pin; important isotopes have been highlighted in red.

In addition to the difference in the burnup between the samples and the assumed material composition in the calculations, two other differences may exist: (1) the measured transmission neutrons were from a slowing-down neutron energy spectrum (2.5-cm slab of polyethylene) versus MCNP neutrons sampled uniformly over the 0-40 
eV energy range and (2) possible differences in the neutron beam geometry (the calculation assumed a cylindrical beam of diameter slightly less than $1.0 \mathrm{~cm}$, whereas the measurement beam geometry is unknown).

It is important to note that the calculated spectrum over the approximately $0.5-40$ $\mathrm{eV}$ energy range looks very much like the measured spectra. The same isotopic depressions are observed with similar depression magnitudes, which means that the calculated spent-fuel compositions contain the right resonant isotopes and the concentrations are reasonable. The good agreement between the measured and calculated transmission spectra for the spent fuels lends some level of confidence and credibility to the MCNP-calculated transmission spectrum, MCNP code models, LANL spent-fuel compositions, ENDF-7 neutron cross sections and, finally, the feasibility studies in this report.

\subsection{PWR Fuel Assembly}

A PWR fuel assembly was chosen as the basis for which all NGSI assay techniques would be evaluated. This particular fuel assembly design is a $17 \times 17$ array with 264 fuel pins and 25 guide tubes. The fuel pins and guide tubes are symmetrically arranged such that each assembly quadrant is a mirror-image of an adjacent quadrant. For NRTA calculations the 25 guide tubes are assumed to be voided or air-filled; the central pin location in the assembly is a guide tube. Fuel pins consist of a $\mathrm{UO}_{2}$ pellet $(0.82 \mathrm{~cm}$ diameter $)$ and a Zircaloy-4 clad tube $(0.95 \mathrm{~cm}$ diameter $)$. The Zircaloy-4 guide tube was assumed to be $1.142 \mathrm{~cm}$ in diameter with a wall thickness of $0.042 \mathrm{~cm}$. The pin pitch was $1.26 \mathrm{~cm}$ and the overall $17 \times 17$ array had the dimensions of $21.42 \times 21.42 \mathrm{~cm}$. The fuel rods had a length $365.76 \mathrm{~cm}(12 \mathrm{ft})$.

Figure 4 shows a cross sectional view of PWR $17 \times 17$ fuel assembly. The 25 large white circles in the assembly are the guide tubes (air-filled), the remaining colored circles represent the fuel pins. Nine (9) vertical lines are drawn over the left half of the assembly and the center row (row 9). In addition, 17 diagonal lines are drawn over the lower half the assembly. At the end of each line is the number of fuel pins in that line. Note that the guide tubes are not counted in the line totals.

The pin totals in Figure 4 range from 1-17. Of the 17 possible vertical lines (only 9 shown in the figure), 3 lines would have 12 pins, 2 lines 14 and 15, and 10 lines 17 . The 3 lines with 12 pins are noteworthy because they are centrally-located, each line containing 5 guide tubes. For the 17 diagonal lines, only 3 lines have more than 12 pins. It can be shown for the PWR $17 \times 17$ assembly that, if the NRTA technique can assay 12 pins in a row in a single integral transmission measurement, then every pin in the assembly can be part of one or more integral transmission measurements; complete assay coverage of all the assembly pins would then be possible. 


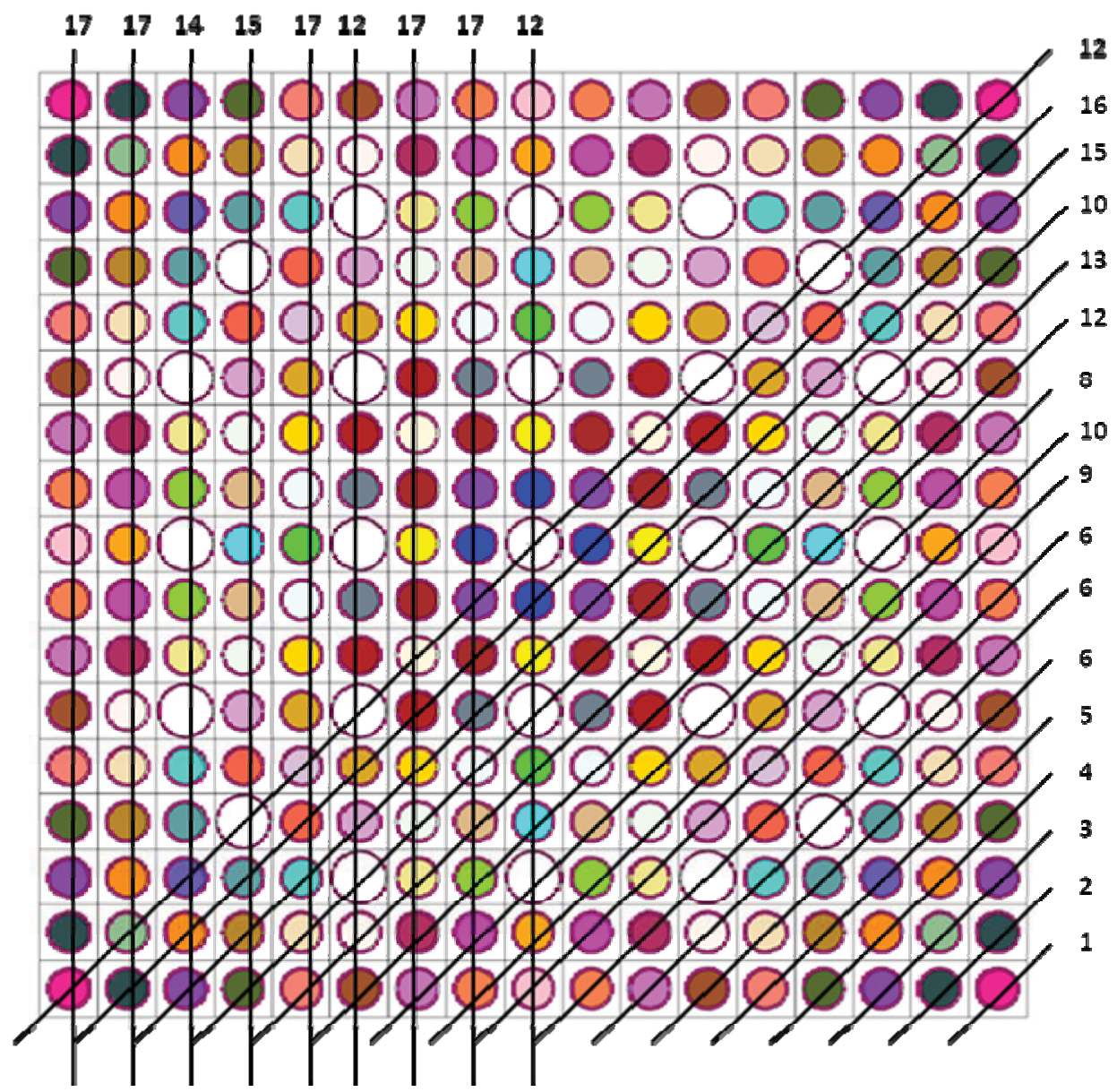

Figure 4 PWR $17 \times 17$ fuel assembly showing number of fuel pins in the vertical columns and diagonal lines.

\section{CONCEPTUAL STUDIES}

This section presents calculated results from several interesting conceptual studies. The calculated results are all based on the MCNP code, models, and neutron cross-section data. The primary goal of these studies was to better understand the basic physics behind the NRTA technique and to preliminarily assess its ability to assay a PWR spent fuel assembly. Parametric variables considered in these studies included spent-fuel isotopics, burnup, cooling time, background neutron emissions, and characteristics of the collimated neutron beam. The results of the conceptual studies here have also provided a preliminary basis upon which a conceptual NRTA system design has begun to emerge. It must be stated that the conceptual studies are not complete. Additional conceptual studies are expected to continue into the future, with the possibility that some of the conceptual studies here will be expanded or re-worked as needed.

The conceptual study results were almost entirely calculated as neutron resonant transmission spectra as a function of the spent-fuel and beam variables. ${ }^{*}$ The

* Note: In the following sections involving simulations with a single fuel pin, the data used for the simulations is from the central region of fuel pin location 36 , a peripheral pin that is in the second location from a corner. 
transmission spectra are all plotted on a linear-linear scale as a detector neutron flux tally or neutron counts as a function of energy or energy bin.

\subsection{Fresh Fuel}

This section presents calculated transmission spectra for fresh $\mathrm{UO}_{2}$ fuel. Fresh fuel is defined as unburned or unirradiated or beginning-of-life fuel. The fresh fuel contains only ${ }^{235} \mathrm{U},{ }^{238} \mathrm{U}$, and oxygen (oxide) as $\mathrm{UO}_{2}$. The initial enrichment was $3.0 \mathrm{wt} \%$ ${ }^{235} \mathrm{U}$. The resonance structure observed in the transmission plots is therefore due only to ${ }^{235} \mathrm{U}$ and ${ }^{238} \mathrm{U}$. Although the $\mathrm{UO}_{2}$ fuel contains a significant amount of oxygen, oxygen has no resonance structure in the $0-100 \mathrm{eV}$ energy range. Similarly, constituents in the Zircaloy-4 clad, mostly natural zirconium but also several other minor elemental constituents including $\mathrm{Sn}, \mathrm{Fe}, \mathrm{Cr}, \mathrm{Ni}, \mathrm{Nb}, \mathrm{C}, \mathrm{O}, \mathrm{Si}, \mathrm{H}$, also have no significant resonance behavior in the $0-100 \mathrm{eV}$ energy range.

As will be seen in the following three figures (Figure 5 - Figure 7), ${ }^{235} \mathrm{U}$ has many more resonances than ${ }^{238} \mathrm{U}$ in the NRTA resonance region; in fact, all the resonance structure/flux depressions here are due to ${ }^{235} \mathrm{U}$ except for the few cases indicated by ${ }^{238} \mathrm{U}$ labels in the figures. The ${ }^{238} \mathrm{U}$ resonances that show up in the $0-40 \mathrm{eV}$ transmission spectra are, however, very deep depressions, basically black to neutrons at the resonance energy due to the high concentration of ${ }^{238} \mathrm{U}$ in the low-enriched commercial fuel. These deep ${ }^{238} \mathrm{U}$ resonance depressions in the transmission spectra will also be seen in spent fuel as well since only a relatively small fraction of the ${ }^{238} \mathrm{U}$ is lost due to transmutation. This is true even for the very high-burnup fuels. The ${ }^{238} \mathrm{U}$ resonances will make dependable energy fiducial markers in the time-energy count data. 


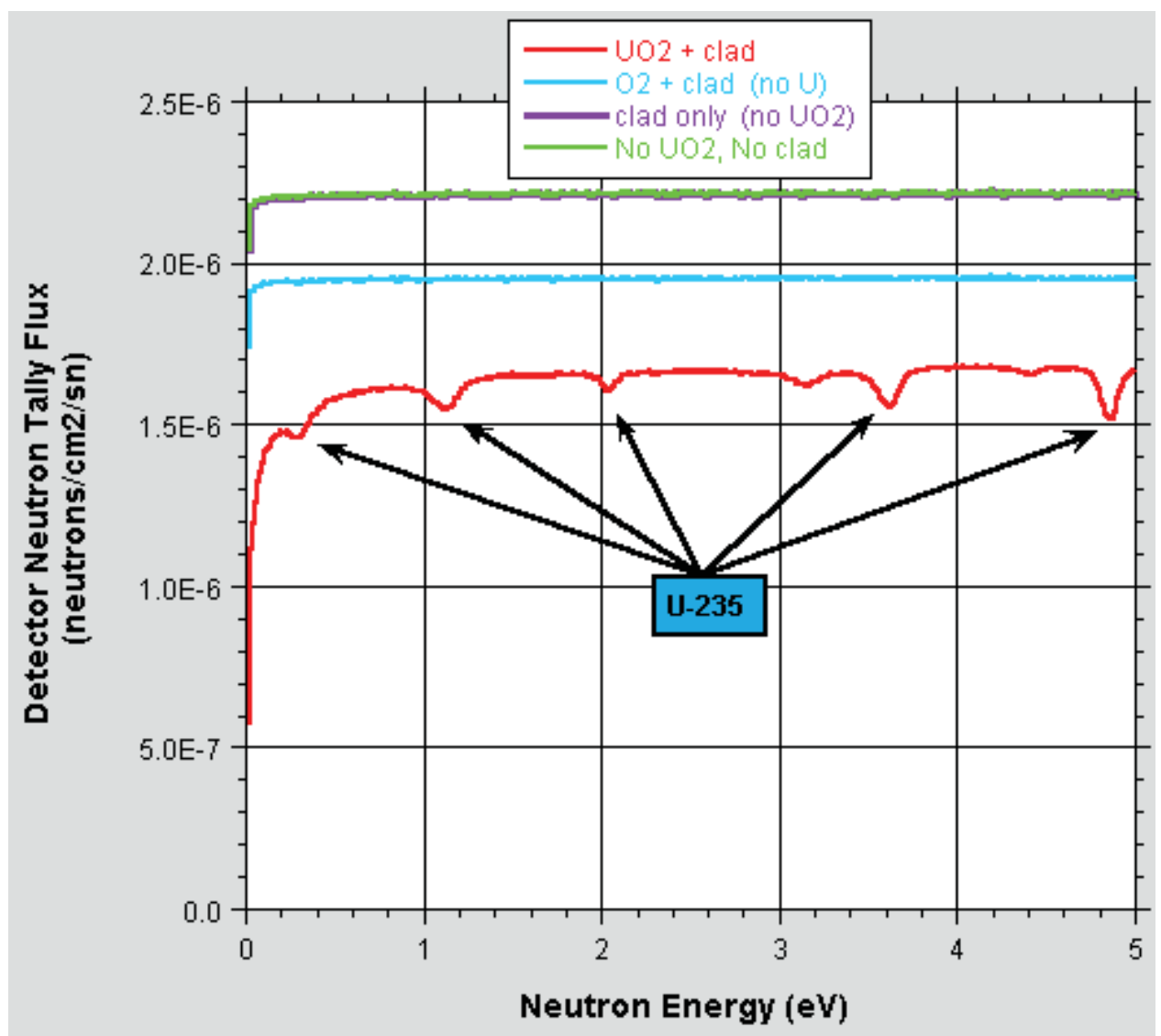

Figure 5 Single pin neutron resonance transmission spectrum from $0-5 \mathrm{eV}$.

Figure 5 through Figure 7 show the calculated transmission spectrum or flux through a single fuel pin with only fresh $\mathrm{UO}_{2}$ fuel. The three figures divide the $0-100 \mathrm{eV}$ energy range into three segments $(0-5 \mathrm{eV}, 0-40 \mathrm{eV}$, and $40-100 \mathrm{eV})$ in order to better resolve the individual resonances in these three regions.

Figure 5 shows the spectrum from $0-5 \mathrm{eV}$. In this figure the red-line corresponds to the neutron transmission flux through the clad and $\mathrm{UO}_{2}$ of a single pin. In this energy region the flux depressions are from ${ }^{235} \mathrm{U}$ resonances only. There are no ${ }^{238} \mathrm{U}$ resonances in this low-energy range. The depressions are relatively shallow due to the low ${ }^{235} \mathrm{U}$ concentration and weak resonances. The blue line shows the transmission flux with the ${ }^{235} \mathrm{U}$ and ${ }^{238} \mathrm{U}$ removed from the oxide fuel matrix, with just the oxide and clad remaining. This confirms that there is no resonance behavior due to oxygen or Zircaloy; hence, there will be no resonance interference from these matrix and clad materials. The purple line shows the transmission spectrum if the oxide is further removed, leaving just the clad. The green line is for the pin completely removed (baseline or unperturbed spectrum). It is interesting to note that the purple and green lines more or less overlay one another, indicating that the clad does not have much effect on the transmitted flux, or that the clad does not scatter the transmission neutrons (primarily because the clad thickness is small and the zirconium scattering cross section is small at these energies). The oxide does significantly scatter neutrons out of the beam, as do the uranium metal atoms. This oxygen-based scattering may be a basis for detecting pin-diversion in cases where a removed pin is not replaced with a surrogate of comparable oxygen content. 
Figure 6 shows the spectrum from $0-40 \mathrm{eV}$. In this figure, the red-line again corresponds to the transmission flux through the clad and $\mathrm{UO}_{2}$ of the single pin. Now there are three prominent ${ }^{238} \mathrm{U}$ resonances, corresponding to the resonant energies of 6.67, 21.0 , and $36.8 \mathrm{eV}$. The rest of the resonance depressions are due to U-235 resonances. The blue, purple, and green lines are same as in Figure 5 and the same conclusions can be drawn.

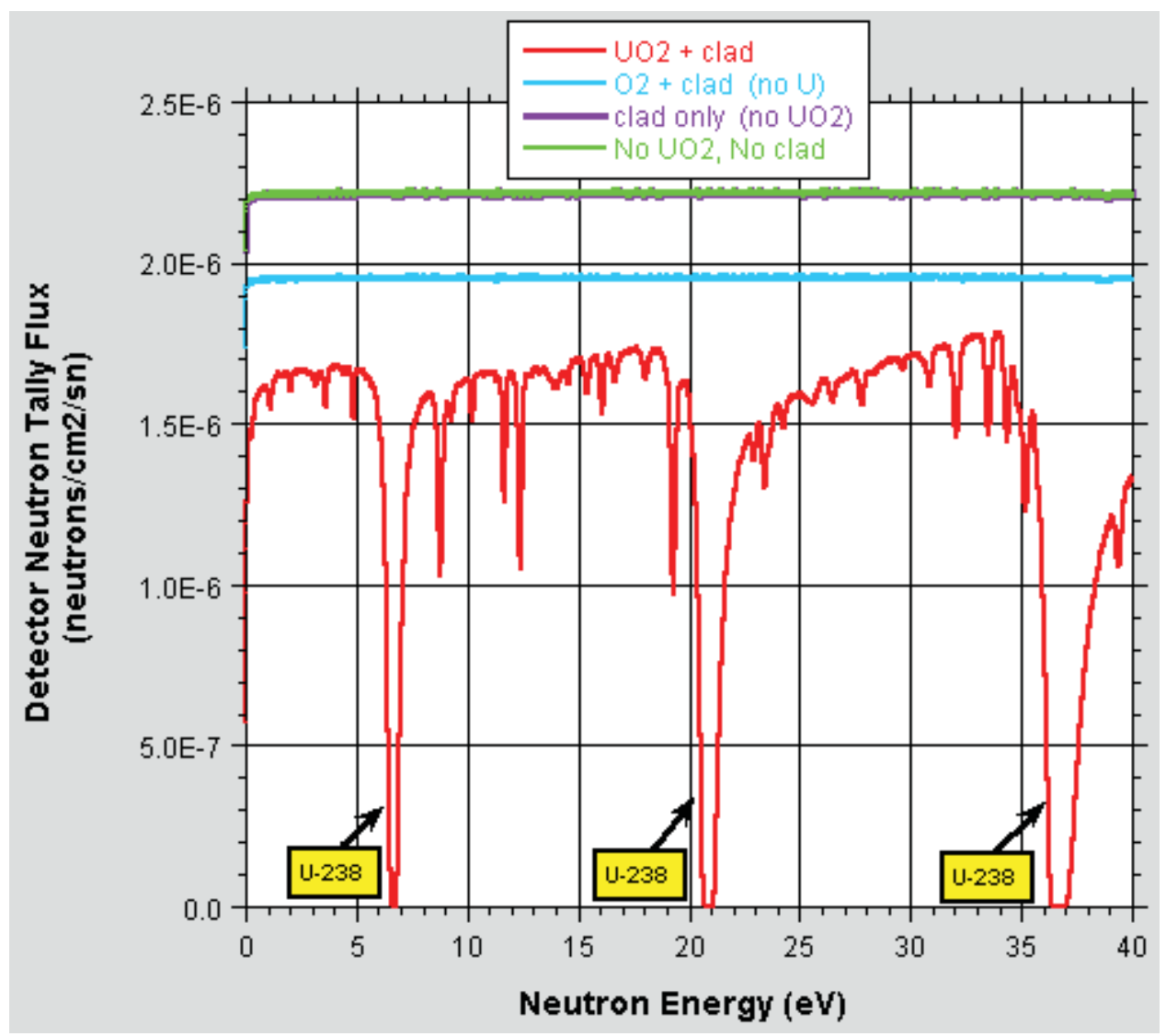

Figure 6 Single pin neutron resonance transmission spectrum from $0-40 \mathrm{eV}$. 
Figure 7 shows just the transmission spectrum (red line) from $40-100 \mathrm{eV}$. In this figure there are two prominent ${ }^{238} \mathrm{U}$ resonances at 66.3 and $81.1 \mathrm{eV}$; the remaining resonance depressions are again due to ${ }^{235} \mathrm{U}$. Even in this higher energy region the clad isotopes and oxygen do not exhibit resonance structure, although this is not shown in the figure.

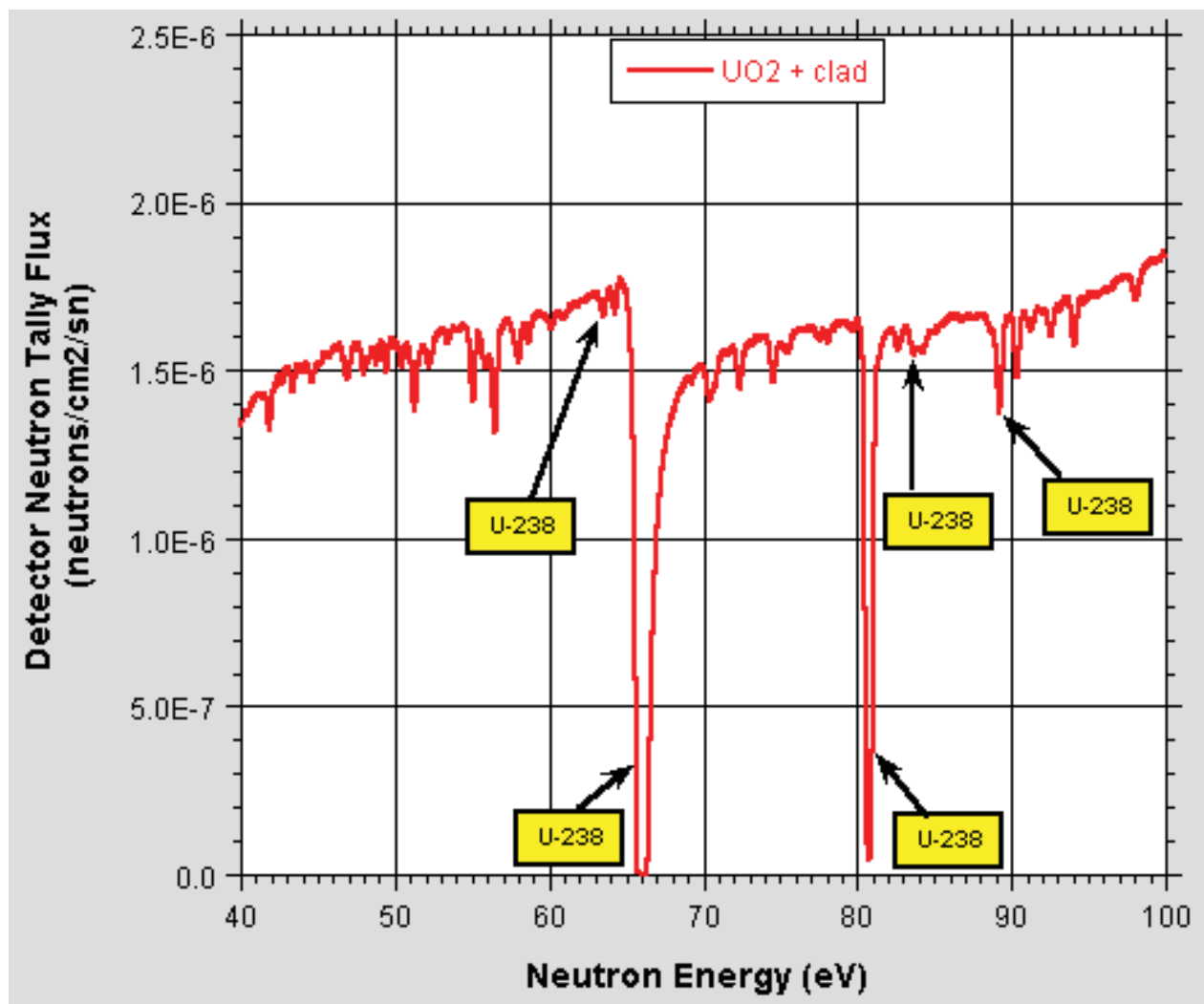

Figure 7 Single pin neutron resonance transmission spectrum from $40-100 \mathrm{eV}$.

\subsection{Fresh Versus Spent}

This section compares calculated transmission spectra between a single fresh $\mathrm{UO}_{2}$ fuel pin and single spent $\mathrm{UO}_{2}$ fuel pin. In addition to the spectral differences, the major actinide and fission-product isotopes are identified in association with their respective resonance depressions. The transmission spectra are compared over the $0-100 \mathrm{eV}$ energy range. The fresh pin contains fresh unburned $\mathrm{UO}_{2}$ with $3.0 \mathrm{wt} \%{ }^{235} \mathrm{U}$ enrichment. The spent fuel pin is $\mathrm{UO}_{2}$ with a burnup of $30 \mathrm{GWD} / \mathrm{MTU}$ and a 1-year cooling time. The spent-fuel $\mathrm{UO}_{2}$ composition is based on a fuel with an initial $3.0 \mathrm{wt} \%{ }^{235} \mathrm{U}$ enrichment.

Figure 8 and Figure 9 show the spectral differences between the two pins over the $0-40$ and $40-100 \mathrm{eV}$ energy range, respectively. The red-line (Fresh $\mathrm{UO}_{2}+$ clad) is the fresh fuel pin and the blue-line (SNF+clad) is the spent fuel pin. For the most part the red and blue curves overlie one another closely. Differences occur mainly in the resonance depressions. In both figures, over the $0-100 \mathrm{eV}$ energy range, the identified ${ }^{238} \mathrm{U}$ resonance depressions remain virtually unchanged. This is due to the fact that the ${ }^{238} \mathrm{U}$ concentration is relatively large and does not deplete significantly with burnup. It 
therefore continues to absorb virtually all the neutrons at the ${ }^{238} \mathrm{U}$ resonance energies in both the fresh and spent fuel cases.

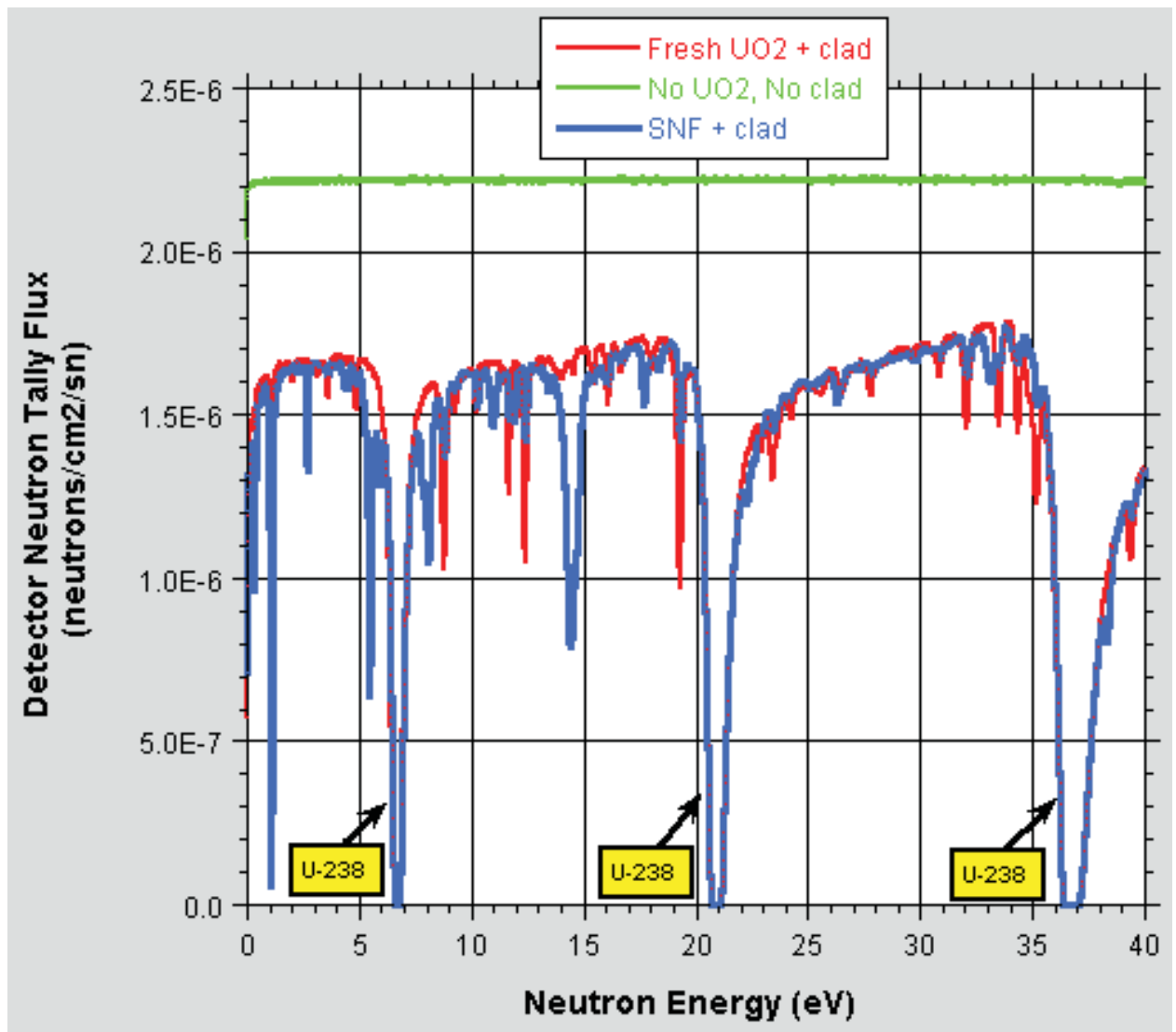

Figure 8 Single pin neutron resonance transmission spectrums for fresh and spent $\mathrm{UO}_{2}$ fuel over the $0-40 \mathrm{eV}$ neutron energy range. 


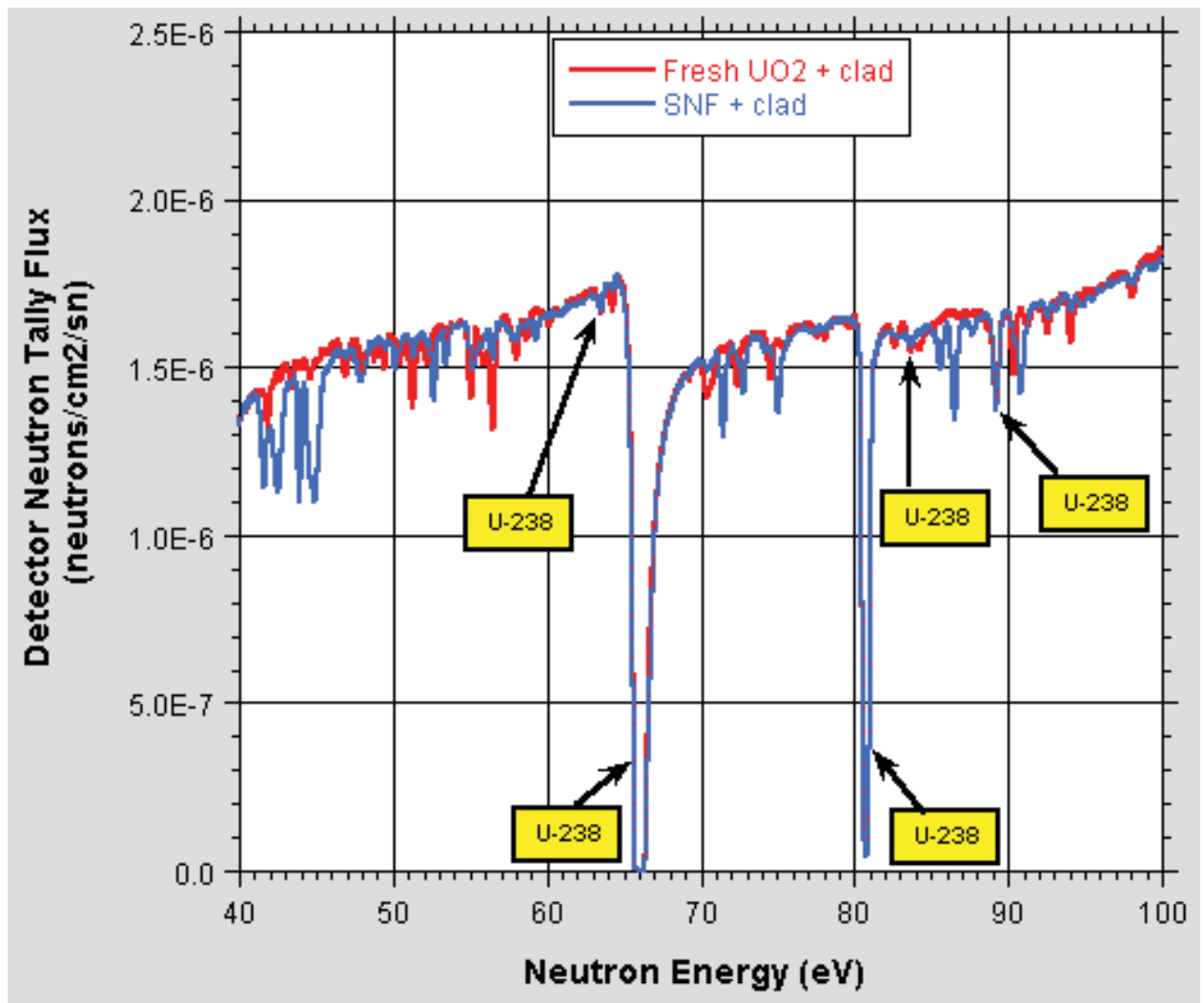

Figure 9 Single pin neutron resonance transmission spectrums for fresh and spent $\mathrm{UO}_{2}$ fuel over the $40-100 \mathrm{eV}$ neutron energy range.

Isotopic resonances other than those of ${ }^{238} \mathrm{U}$ will be identified in detail in the following five figures (Figure 10 - Figure 14). These other resonance depressions, with the exception of those due to ${ }^{235} \mathrm{U}$, appear in the spent fuel pin due to the buildup of transuranics and fission products with burnup. The ${ }^{235} \mathrm{U}$ resonances are easily identifiable, however, because they behave differently the ${ }^{235} \mathrm{U}$ concentration depletes significantly over the course of the $30 \mathrm{GWD} / \mathrm{MTU}$ burnup and the spent-fuel blue-line depressions show a reduction in magnitude (less deep) relative to the red-line depressions. Red-line depressions deeper than the blue are due to ${ }^{235} \mathrm{U}$ resonances. The green-line (no $\mathrm{UO}_{2}$, no clad) is again the transmission spectrum with no pin, showing the relative amount of neutron out-scatter and absorption that can be expected for the fresh and spent fuel pins.

Figure 10 through Figure 14 are the same as Figure 8 but sub-divide the energy range into finer increments in order to better identify the major individual fresh- and spent-fuel isotopic resonance depressions. Figure 10 covers the energy range for $0-5 \mathrm{eV}$, Figure 11 5-10 eV, Figure 12 10-15 eV, Figure 13 15-20 eV, and Figure 14 the larger 20$40 \mathrm{eV}$ energy range. 
Figure 10 shows the two resonance transmission spectra for the $0-5 \mathrm{eV}$ energy range. In this energy range, there are three important plutonium resonances, namely, ${ }^{239} \mathrm{Pu}(0.3 \mathrm{eV}),{ }^{240} \mathrm{Pu}(1.05 \mathrm{eV})$, and ${ }^{242} \mathrm{Pu}(2.65 \mathrm{eV})$. The fission product ${ }^{145} \mathrm{Nd}$ produces a resonance depression at $4.35 \mathrm{eV}$. Neodymium- 145 is a stable nuclide and will increase in concentration in the spent fuel with increasing burnup. It will be an important spentfuel burnup indicator. In addition, two small ${ }^{235} \mathrm{U}$ resonance depressions are observed at 3.6 and $4.8 \mathrm{eV}$. Note the reduction in magnitude of these two ${ }^{235} \mathrm{U}$ depressions for the spent fuel relative to the fresh fuel.

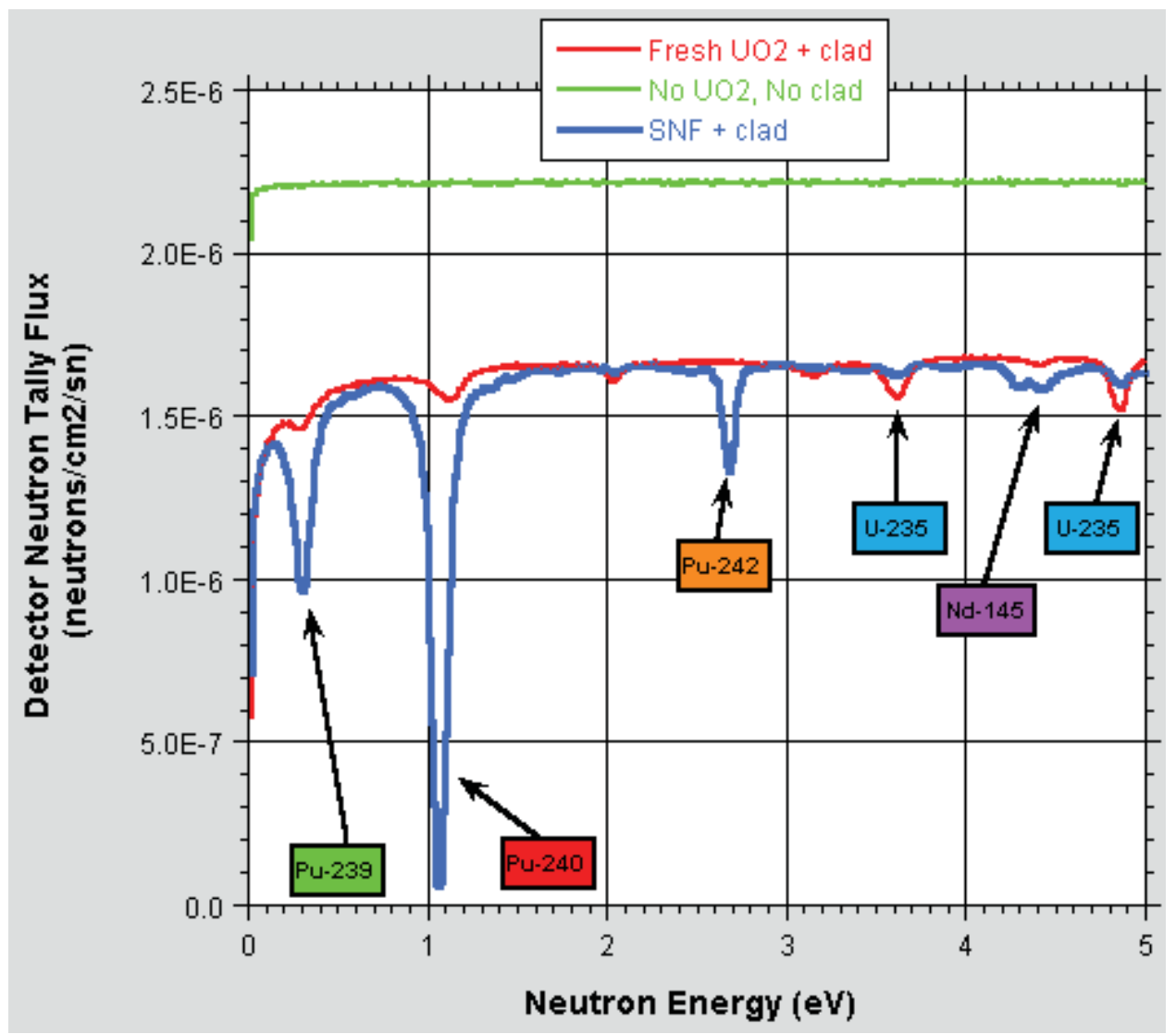

Figure 10 Single pin neutron resonance transmission spectrums for fresh and spent $\mathrm{UO}_{2}$ fuel over the 0-5 $\mathrm{eV}$ neutron energy range. 
Figure 11 shows the two resonance transmission spectra for the 5-10 eV energy range. In this energy range, there are three important uranium resonances: ${ }^{235} \mathrm{U}(8.8 \mathrm{eV})$, ${ }^{236} \mathrm{U}(5.45 \mathrm{eV})$, and ${ }^{238} \mathrm{U}(6.67 \mathrm{eV})$. An overlapping resonance depression at approximately $5.9 \mathrm{eV}$ is observed for the two fission products ${ }^{133} \mathrm{Cs}$ and ${ }^{99} \mathrm{Tc}$. Cesium133 is a stable nuclide; ${ }^{99} \mathrm{Tc}$ (ground state) is radioactive but has a relatively long half-life (213,000 years). Hence, both ${ }^{133} \mathrm{Cs}$ and ${ }^{99} \mathrm{Tc}$ concentrations in the spent fuel should increase with burnup and act as an important burnup indicator as well. The fission product ${ }^{152} \mathrm{Sm}$ is also observed in this energy range as a depression at $8.1 \mathrm{eV}$. Samarium152 is a stable nuclide with an appreciable neutron capture resonance at $8.1 \mathrm{eV}$ that produces a resonance depression with increasing magnitude with burnup, again a potentially important fission-product burnup indicator.

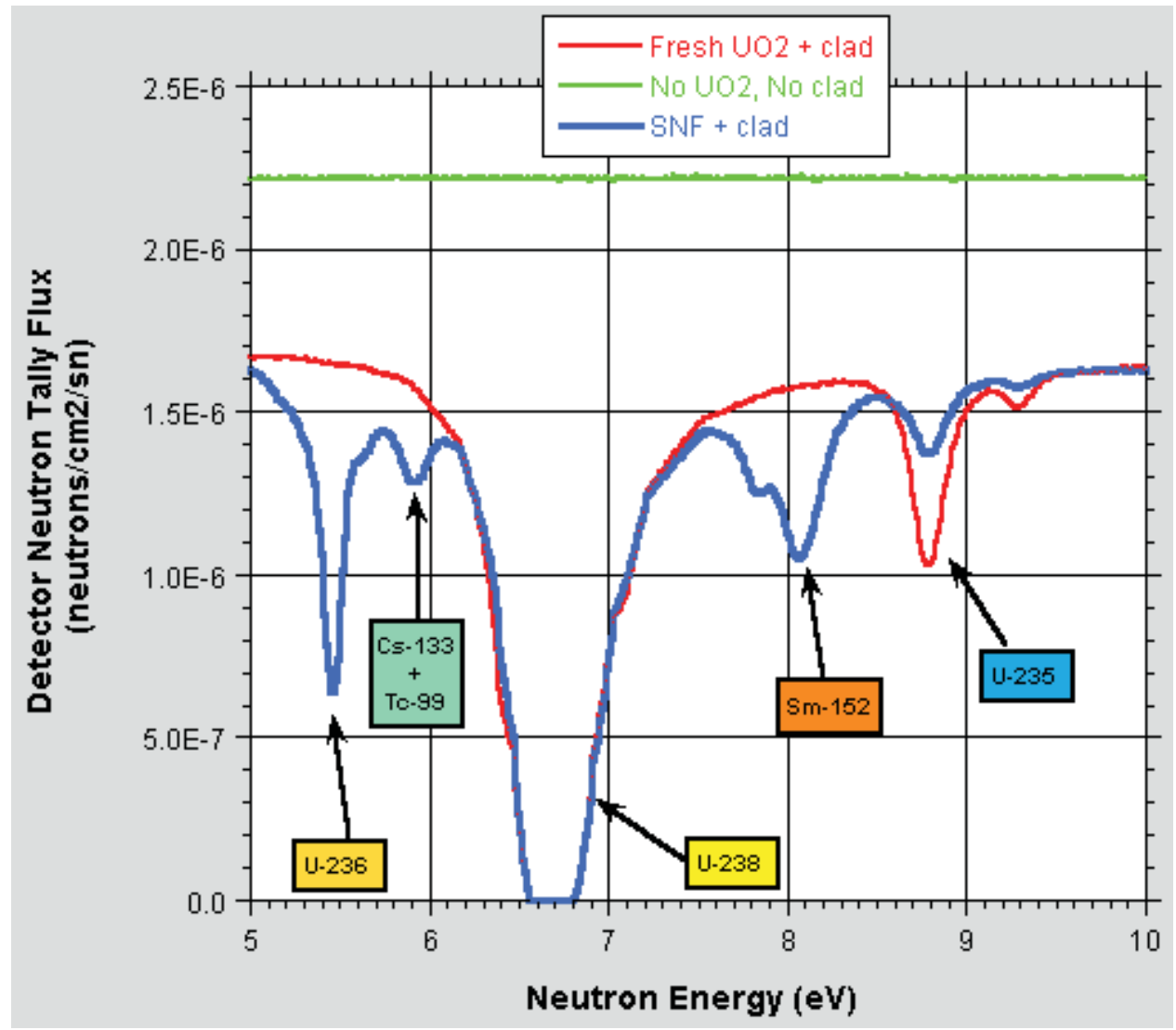

Figure 11 Single pin neutron resonance transmission spectrums for fresh and spent $\mathrm{UO}_{2}$ fuel over the 5-10 eV neutron energy range. 
Figure 12 shows the two resonance transmission spectra for the 10-15 eV energy range. In this energy range there are two small ${ }^{239} \mathrm{Pu}$ resonance depressions at 10.9 and $11.9 \mathrm{eV}$, two ${ }^{235} \mathrm{U}$ resonances at 11.65 and $12.4 \mathrm{eV}$, one small ${ }^{238} \mathrm{U}$ resonance at 10.25 $\mathrm{eV}$, one small ${ }^{241} \mathrm{Pu}$ resonance at $13.4 \mathrm{eV}$, and a very large and significant fission product resonance depression from ${ }^{131} \mathrm{Xe}$ at $14.4 \mathrm{eV}$. Xenon-131 is a stable fission-product nuclide and can also be an important spent fuel indicator. Perhaps more importantly, this isotope may also serve as a difficult-to-replicate pin-diversion indicator. Xenon is a noble gas; reprocessing of spent fuel will release the xenon gas from the fuel composition. Reincorporating xenon gas in the correct amount back into a diversion spent fuel pin composition would be very difficult. The NRTA technique should be able to readily detect diverted spent fuel pins missing Xe-131 (e.g., using depleted uranium polluted with other radioactive fission products).

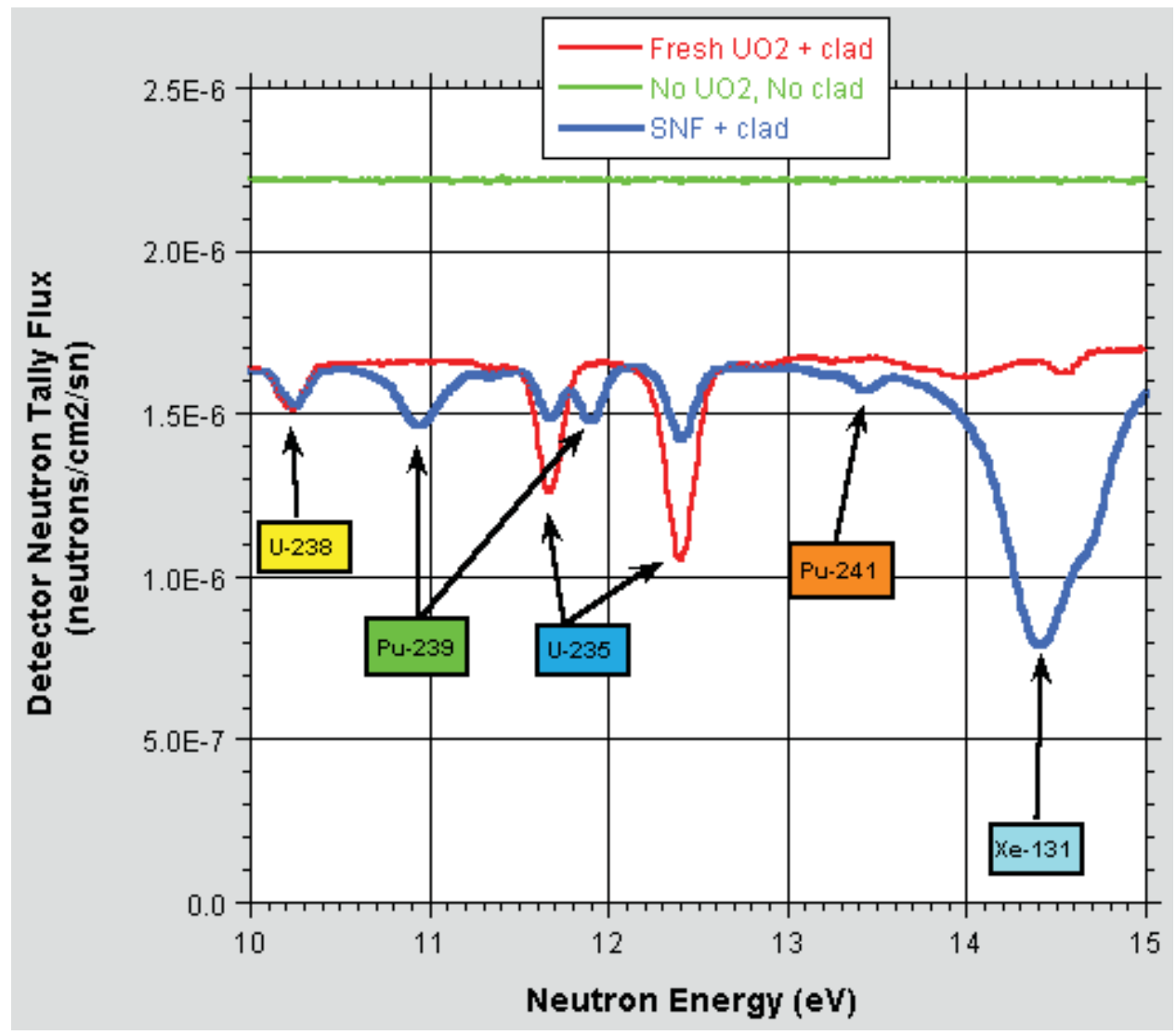

Figure 12 Single pin neutron resonance transmission spectrums for fresh and spent $\mathrm{UO}_{2}$ fuel over the $10-15 \mathrm{eV}$ neutron energy range. 
Figure 13 shows the two resonance transmission spectra for the 15-20 eV energy range. In this energy range there is one small ${ }^{239} \mathrm{Pu}$ resonance depressions at $17.6 \mathrm{eV}$ and one large ${ }^{235} \mathrm{U}$ resonance at $19.4 \mathrm{eV}$. There appear to be no other notable resonance depressions in this energy range.

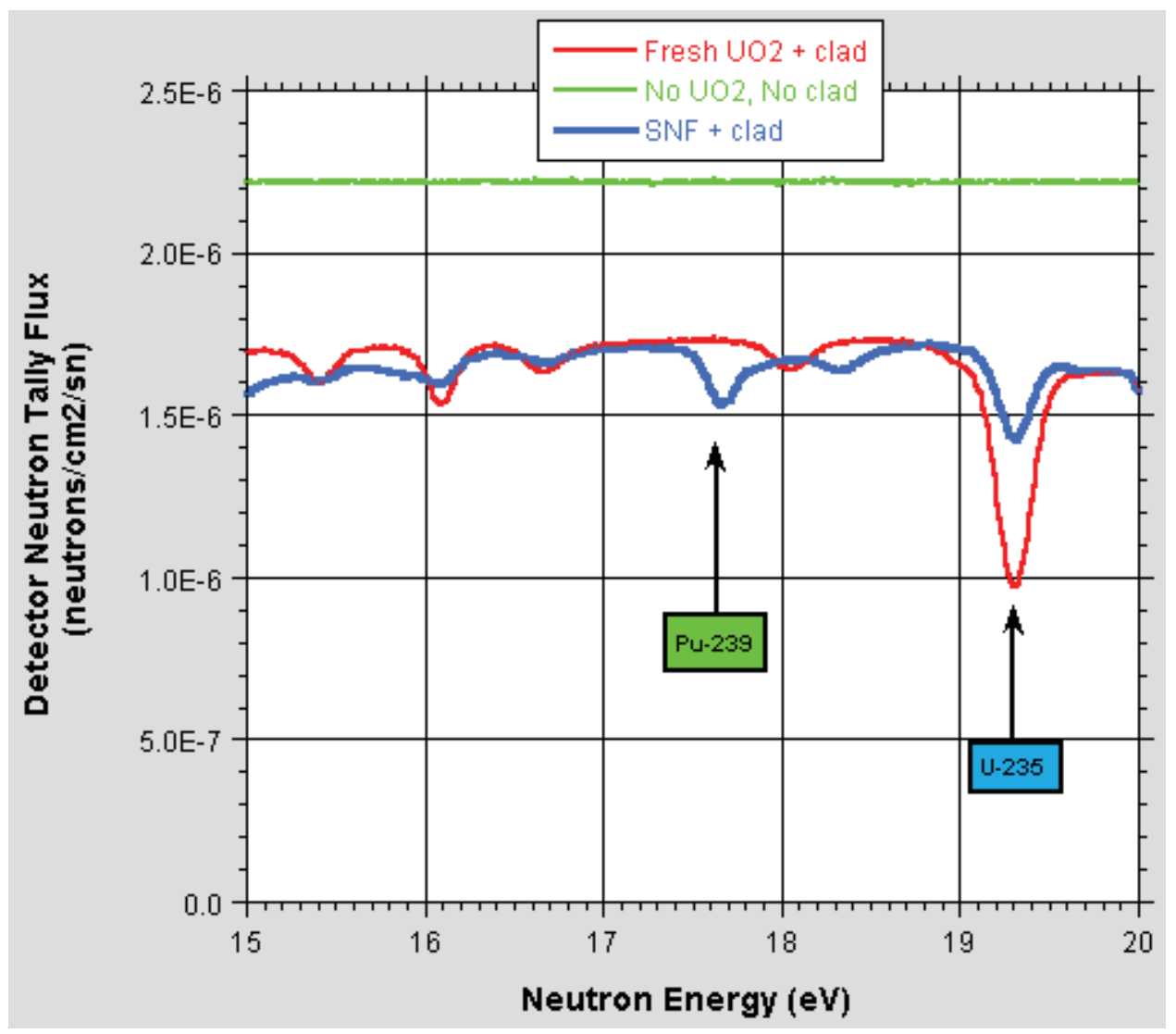

Figure 13 Single pin neutron resonance transmission spectrums for fresh and spent $\mathrm{UO}_{2}$ fuel over the 15-20 eV neutron energy range. 
Figure 14 shows the two resonance transmission spectra for the 20-40 eV energy range. In this range there are four ${ }^{235} \mathrm{U}$ resonance depressions at approximately 32.0, $33.5,34.4$, and $35.2 \mathrm{eV}$. Also, two large ${ }^{238} \mathrm{U}$ resonances appear at 20.9 and $36.8 \mathrm{eV}$. Again, there appear to be no other notable resonance depressions in this energy range.

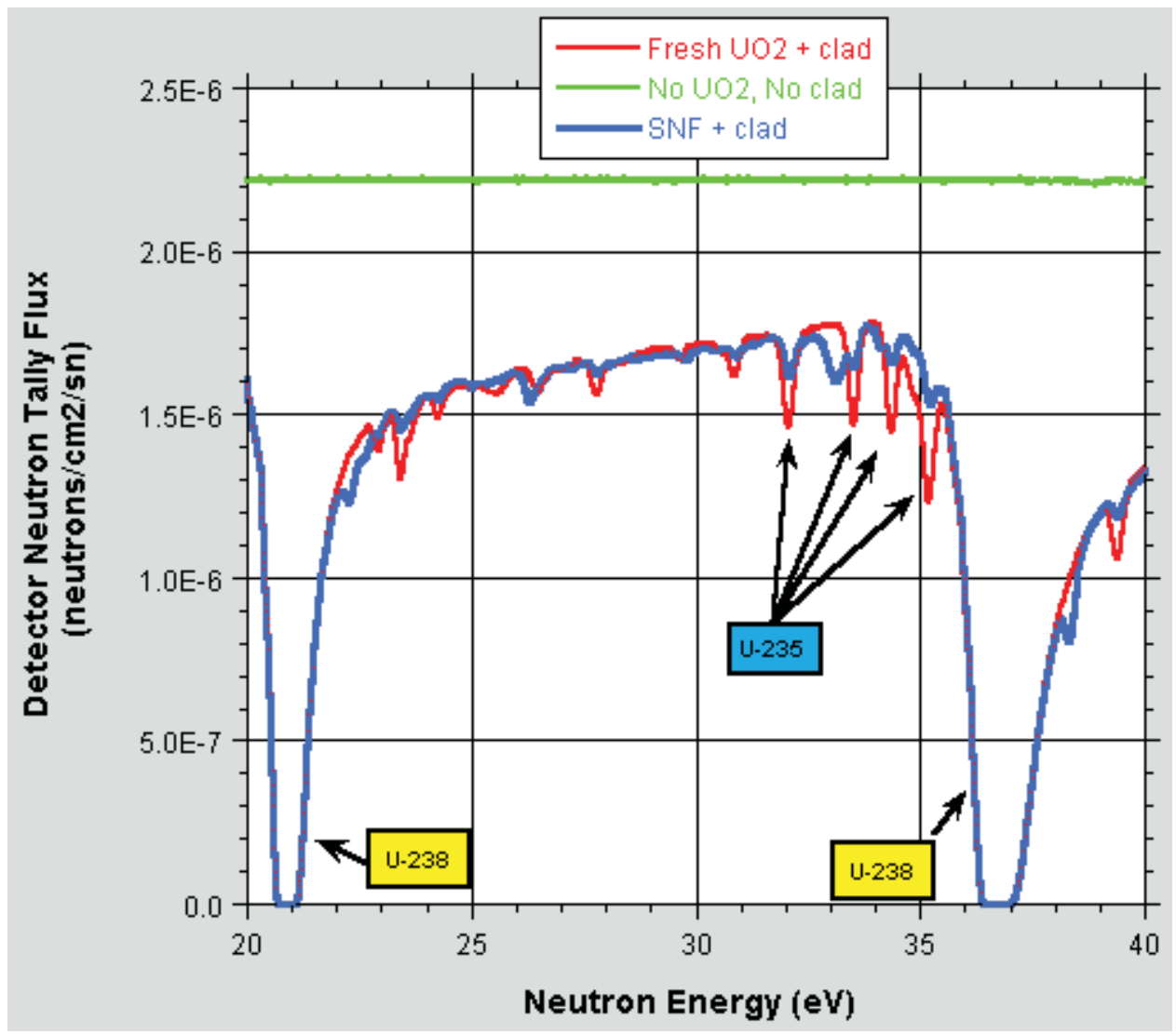

Figure 14 Single pin neutron resonance transmission spectrums for fresh and spent $\mathrm{UO}_{2}$ fuel over the 20-40 eV neutron energy range.

It is interesting to note that a useful transmission neutron-energy range for the NRTA technique may be $0.1-15 \mathrm{eV}$. Above $15 \mathrm{eV}$ in the $15-40 \mathrm{eV}$ range, for example, there appears to be mostly small and redundant resonances of ${ }^{235} \mathrm{U},{ }^{238} \mathrm{U},{ }^{238} \mathrm{U},{ }^{239} \mathrm{Pu}$, ${ }^{240} \mathrm{Pu},{ }^{241} \mathrm{Pu}$, and ${ }^{242} \mathrm{Pu}$. Restricting the NRTA transmission energy range to $0.1-15 \mathrm{eV}$ will reduce the required length of the flight tube, allow assays of all the plutonium isotopes plus ${ }^{131} \mathrm{Xe}$, and help to make the NRTA system footprint smaller.

\subsection{Burnup}

This section compares calculated transmission spectra as a function of burnup. Spent-fuel composition data were taken directly from the LANL 64 spent fuel library data for $3.0 \mathrm{wt} \%{ }^{235} \mathrm{U}$ initial enrichment, 15, 30, 45, and $60 \mathrm{GWD} / \mathrm{MTU}$ burnups, and a 5year cooling time. These transmission spectra are again for a single PWR fuel pin.

Figure 15 shows the neutron transmission spectra for the four different burnups $(15,30,45$, and $60 \mathrm{GWD} / \mathrm{MTU})$ over the $0-40 \mathrm{eV}$ neutron energy range. Resonance depressions due to specific actinide and fission-product isotopes are not identified in the 
following figures in order to more clearly see the resonance depression differences with burnup. Isotopes corresponding to specific resonances can be determined by using Figure 8 through Figure 14.

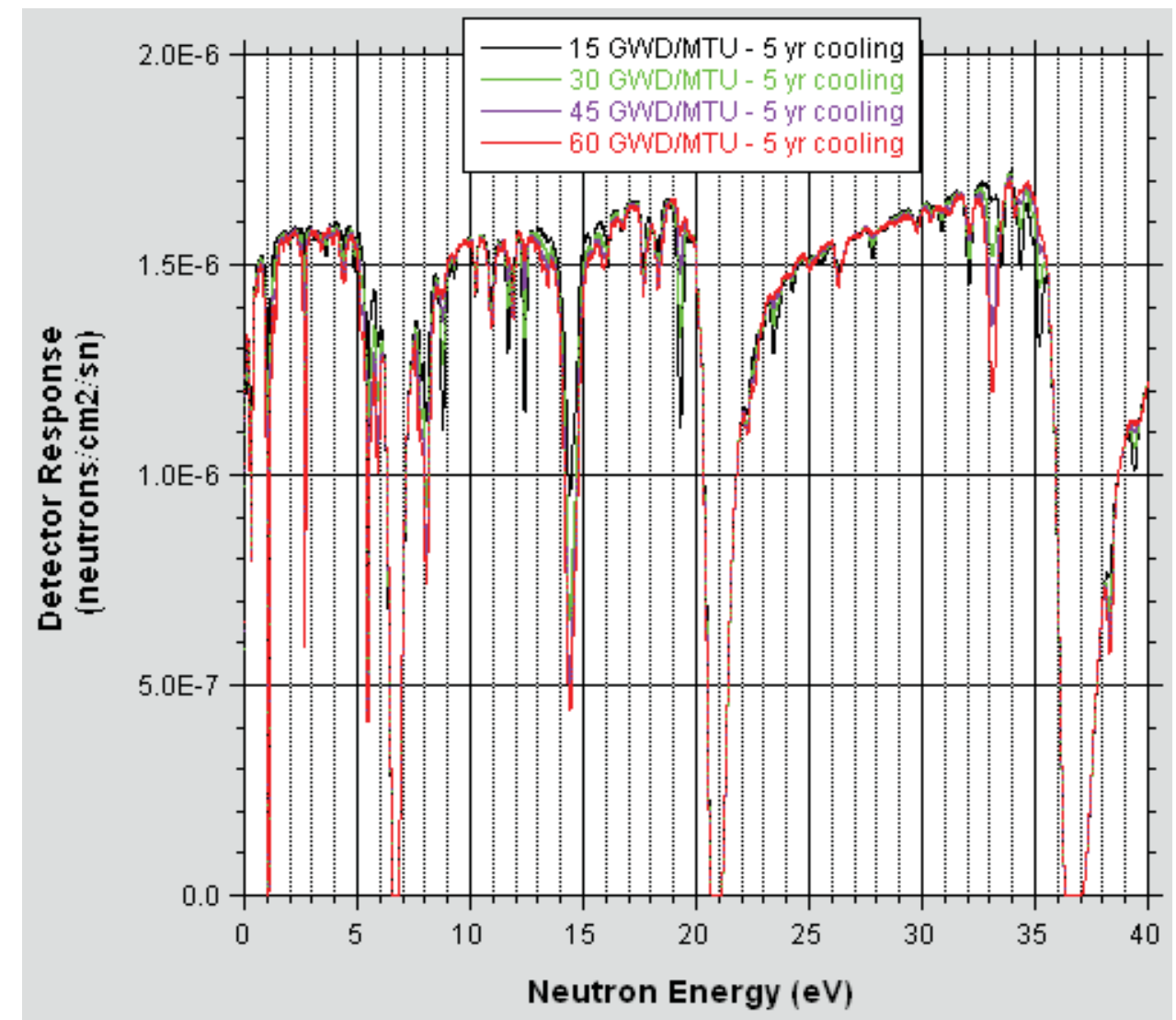

Figure 15 Transmission spectra as a function of burnup over the 0-40 eV energy range.

The following five figures, Figure 16 through Figure 20, sub-divide the transmission spectra in Figure 15 into finer energy intervals in order to show more detail in the resonance depression behavior as a function of burnup. 
Figure 16 shows the resonance transmission spectra as a function of burnup for the $0-2 \mathrm{eV}$ energy range. In this range, the two largest resonance depressions, ${ }^{239} \mathrm{Pu}$ at 0.3 $\mathrm{eV}$ and ${ }^{240} \mathrm{Pu}$ at $1.07 \mathrm{eV}$, are clearly visible. Both depressions increase in magnitude (deepen) with increasing burnup. The deepening is most pronounced for burnups of 15 and $30 \mathrm{GWD} / \mathrm{MTU}$. For burnups greater than $30 \mathrm{GWD} / \mathrm{MTU}$ the resonance depression does not change much, which is indicative of ${ }^{239} \mathrm{Pu}$ and ${ }^{240} \mathrm{Pu}$ reaching maximum, or saturation concentrations. These two plutonium resonances are expected to be important NRTA resonances, resonances that will be measured by the NRTA technique in order to estimate plutonium content in spent fuel pins and assemblies.

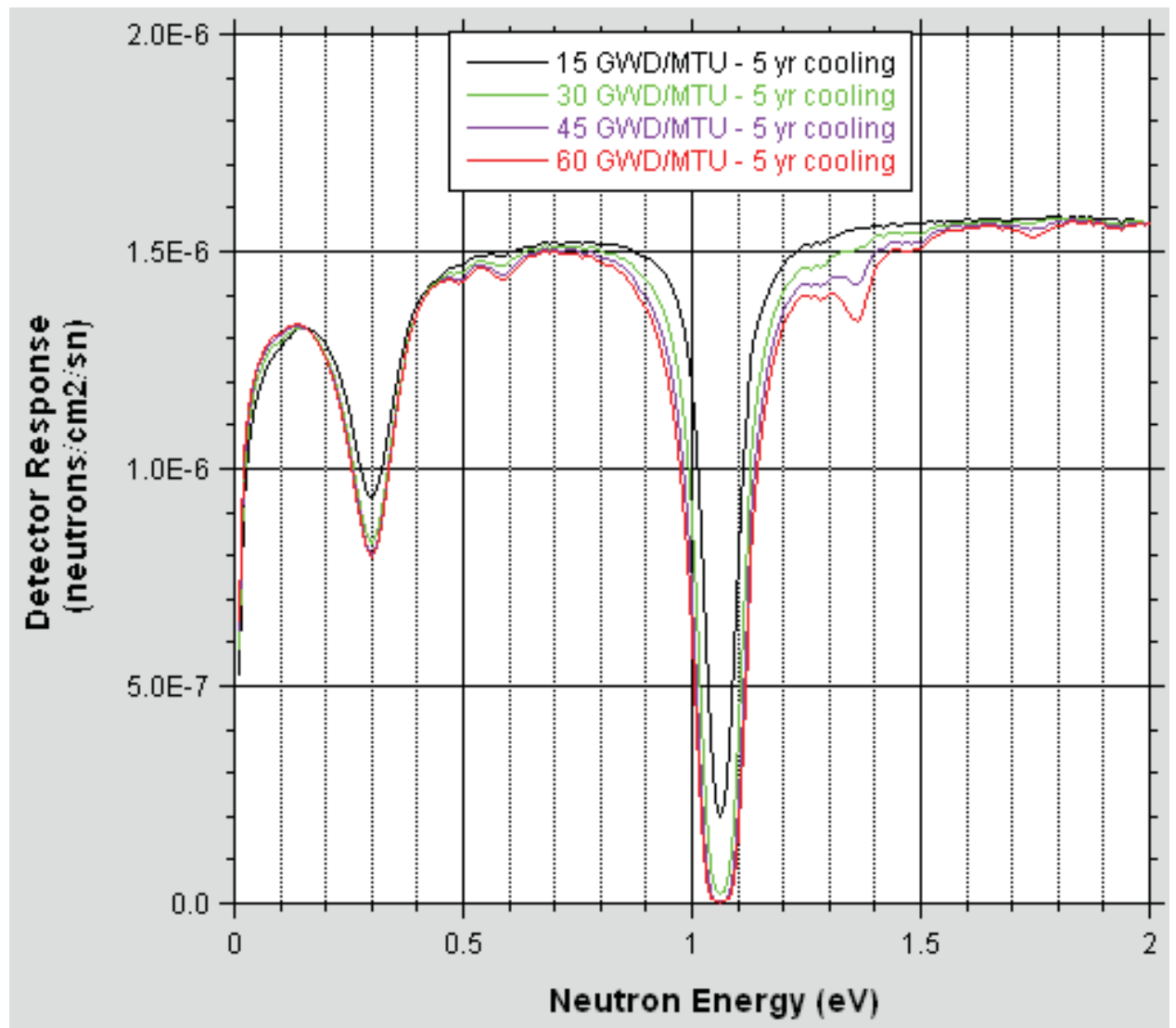

Figure 16 Transmission spectra as a function of burnup over the $0-2 \mathrm{eV}$ energy range. 
Figure 17 shows the resonance transmission spectra as a function of burnup for the $0-10 \mathrm{eV}$ energy range. In this range five additional large resonance depressions are clearly visible, namely ${ }^{242} \mathrm{Pu}$ at $2.65 \mathrm{eV},{ }^{236} \mathrm{U}$ at $5.45 \mathrm{eV},{ }^{133} \mathrm{Cs} /{ }^{99} \mathrm{Tc}$ at $5.9 \mathrm{eV},{ }^{152} \mathrm{Sm}$ at $8.1 \mathrm{eV}$, and ${ }^{235} \mathrm{U}$ at $8.8 \mathrm{eV}$. With the exception of ${ }^{235} \mathrm{U}$, the magnitude of these resonance depressions increases (deepens) proportionally with burnup. These isotopic resonance depressions are sensitive to burnup, do not saturate over the 0-60 GWD/MTU burnup range, and should provide very measurable signals for the NRTA technique. The ${ }^{235} \mathrm{U}$ resonance depression decreases in magnitude with increasing burnup, as noted previously. Ratios of the peak flux depressions for ${ }^{242} \mathrm{Pu},{ }^{236} \mathrm{U},{ }^{133} \mathrm{Cs} /{ }^{99} \mathrm{Tc}$, or ${ }^{152} \mathrm{Sm}$ to ${ }^{235} \mathrm{U}$ should provide useful burnup estimates for comparison to the high-fidelity burnup calculations.

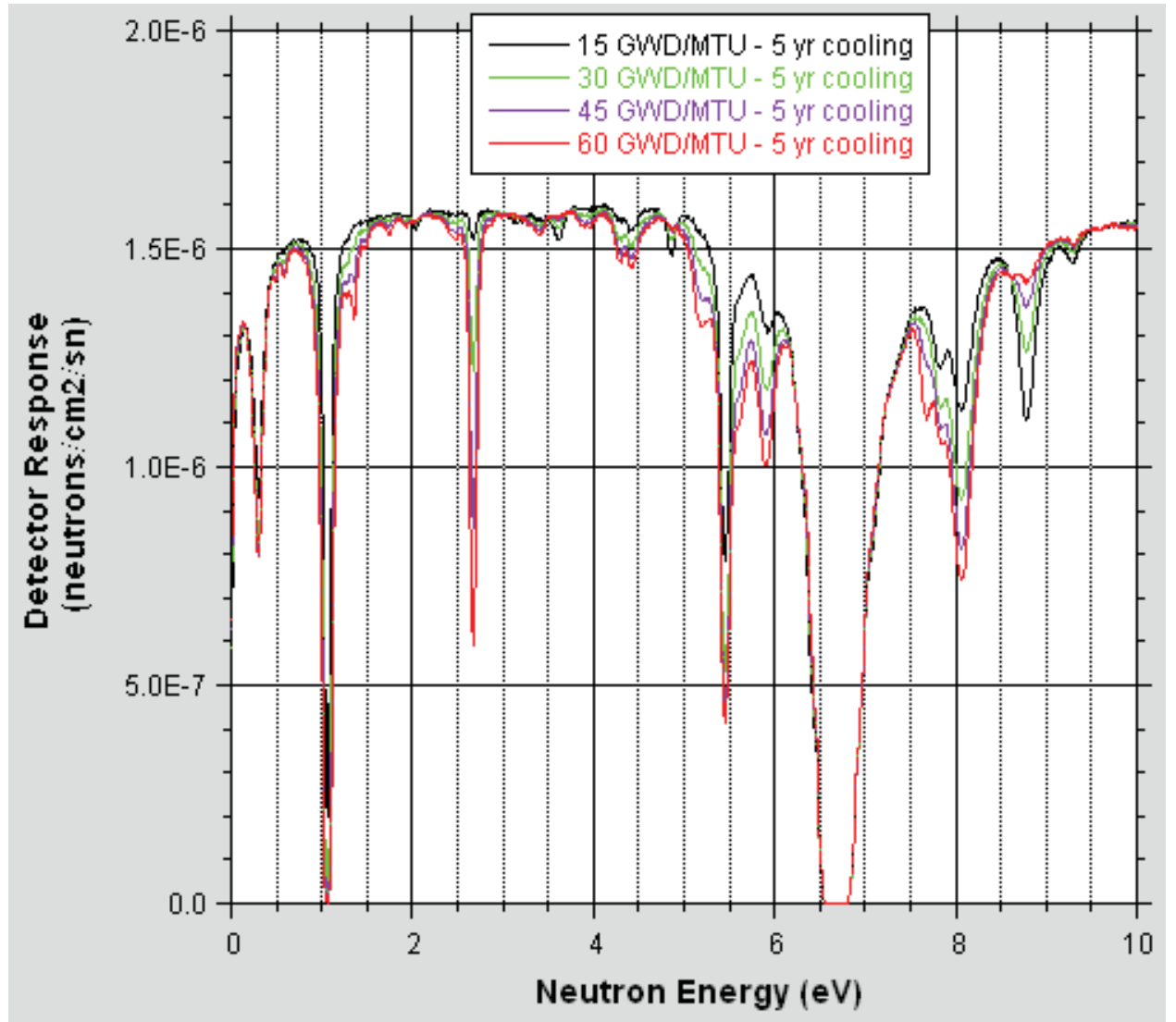

Figure 17 Transmission spectra as a function of burnup over the 0-10 eV energy range. 
Figure 18 shows resonance transmission spectra as a function of burnup within the $10-20 \mathrm{eV}$ energy range. In this range the largest resonance depression is ${ }^{131} \mathrm{Xe}$ at 14.4 $\mathrm{eV}$. This depression increases proportionally with burnup and begins to saturate above $45 \mathrm{GWD} / \mathrm{MTU}$. The ${ }^{241} \mathrm{Pu}$ depression at $13.4 \mathrm{eV}$ remains small but does increase proportionally with burnup. The two ${ }^{239} \mathrm{Pu}$ depressions at 10.9 and $11.9 \mathrm{eV}$ remain relatively small and appear to saturate, as expected, for burnups greater than 30 GWD/MTU. The ${ }^{241} \mathrm{Pu}$ depression at $13.4 \mathrm{eV}$ increases slightly with burnup but is still relatively shallow even at $60 \mathrm{GWD} / \mathrm{MTU}$. The three ${ }^{235} \mathrm{U}$ depressions at $11.65,12.4$, and $19.4 \mathrm{eV}$ all tend to decrease in magnitude proportional to burnup and nearly disappear at $60 \mathrm{GWD} / \mathrm{MTU}$. The small ${ }^{238} \mathrm{U}$ depression at $10.25 \mathrm{eV}$ remains virtually fixed. There are several smaller depressions which need to be identified and could provide additional burnup signature information.

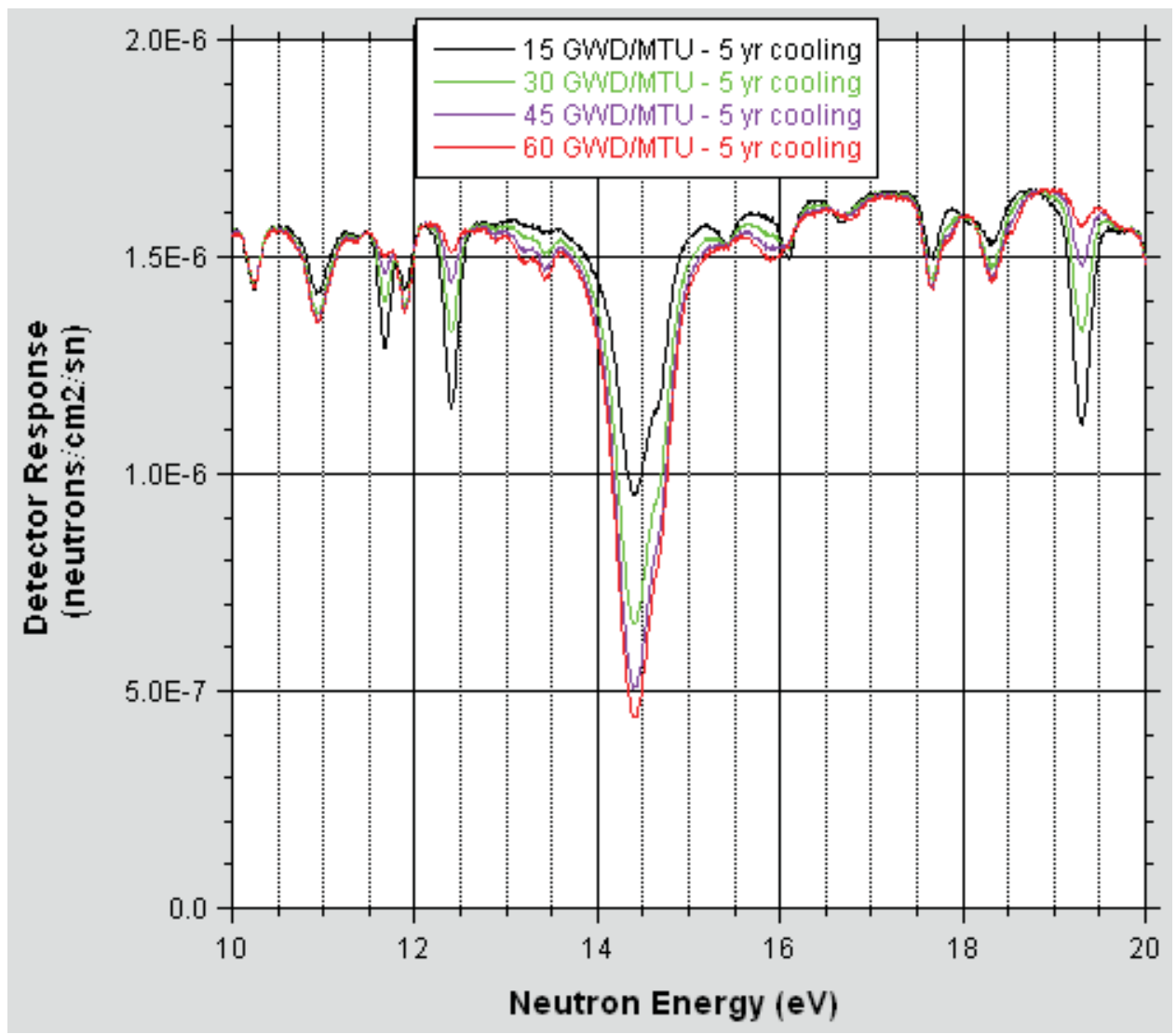

Figure 18 Transmission spectra as a function of burnup over the 10-20 eV energy range. 
Figure 19 shows the resonance transmission spectra as a function of burnup within the $20-30 \mathrm{eV}$ energy range. In this range the largest resonance depression is ${ }^{238} \mathrm{U}$ at $20.9 \mathrm{eV}$. Also, there are several small ${ }^{235} \mathrm{U}$ resonance depressions (clearly identifiable) as well as several other small actinide depressions not yet identified. In general, this energy region does not appear to provide substantial new isotopic resonance information.

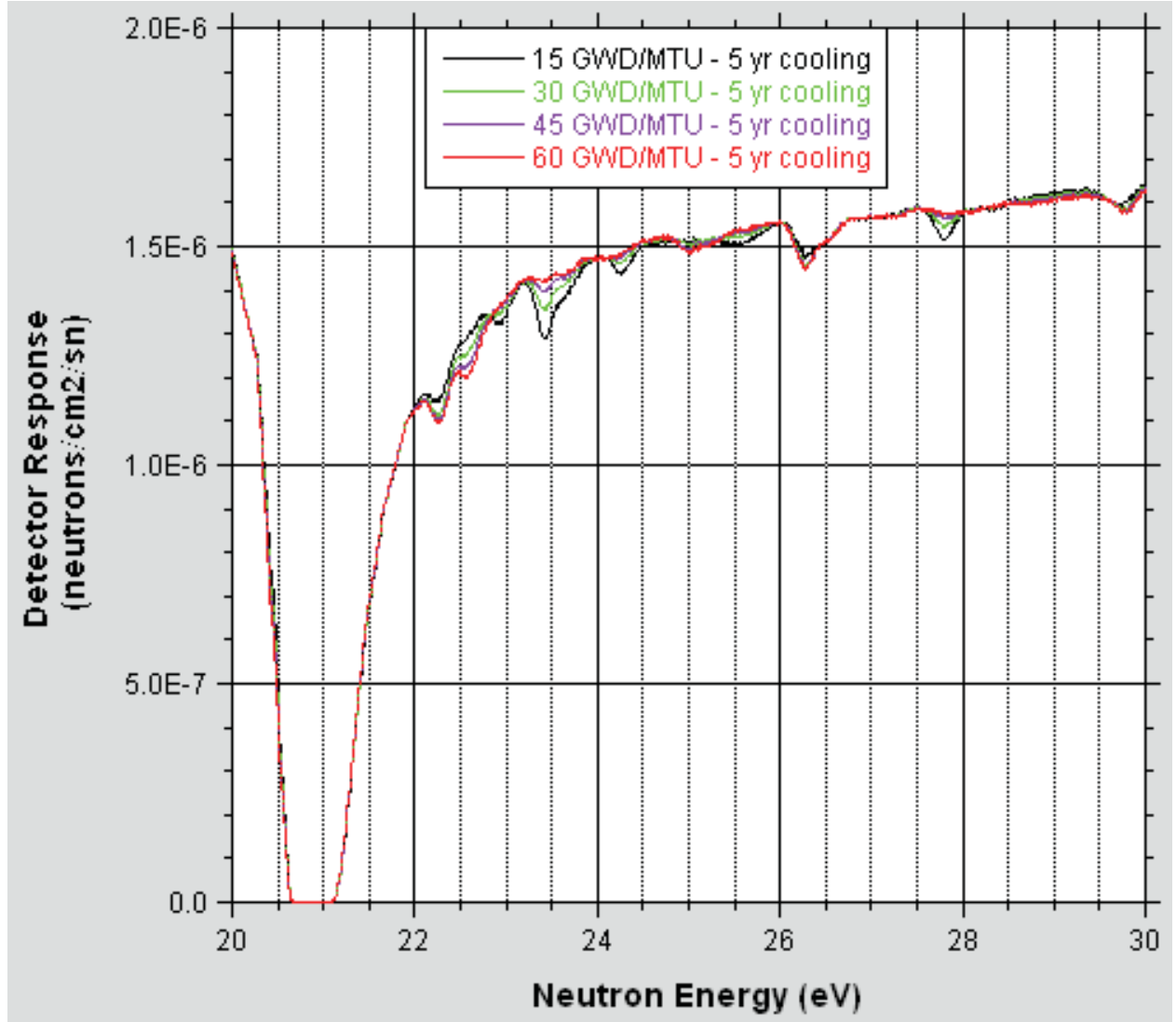

Figure 19 Transmission spectra as a function of burnup over the 20-30 eV energy range. 
Figure 20 shows the resonance transmission spectra as a function of burnup for the $30-40 \mathrm{eV}$ energy range. In this range the largest resonance depression is again ${ }^{238} \mathrm{U}$ at $36.8 \mathrm{eV}$. Also, there are five ${ }^{235} \mathrm{U}$ resonances at approximately $32.1,33.5,34.4,35.2$, and $39.4 \mathrm{eV}$. These five small depressions decrease in magnitude proportionally with burnup and virtually disappear at $60 \mathrm{GWD} / \mathrm{MTU}$. A potentially useful but as yet unidentified resonance depression occurs at approximately $33.15 \mathrm{eV}$. This depression increases proportionally with burnup and does not saturate, nor hint of saturation, even up to 60 GWD/MTU. Future studies will identify the isotope causing this depression.

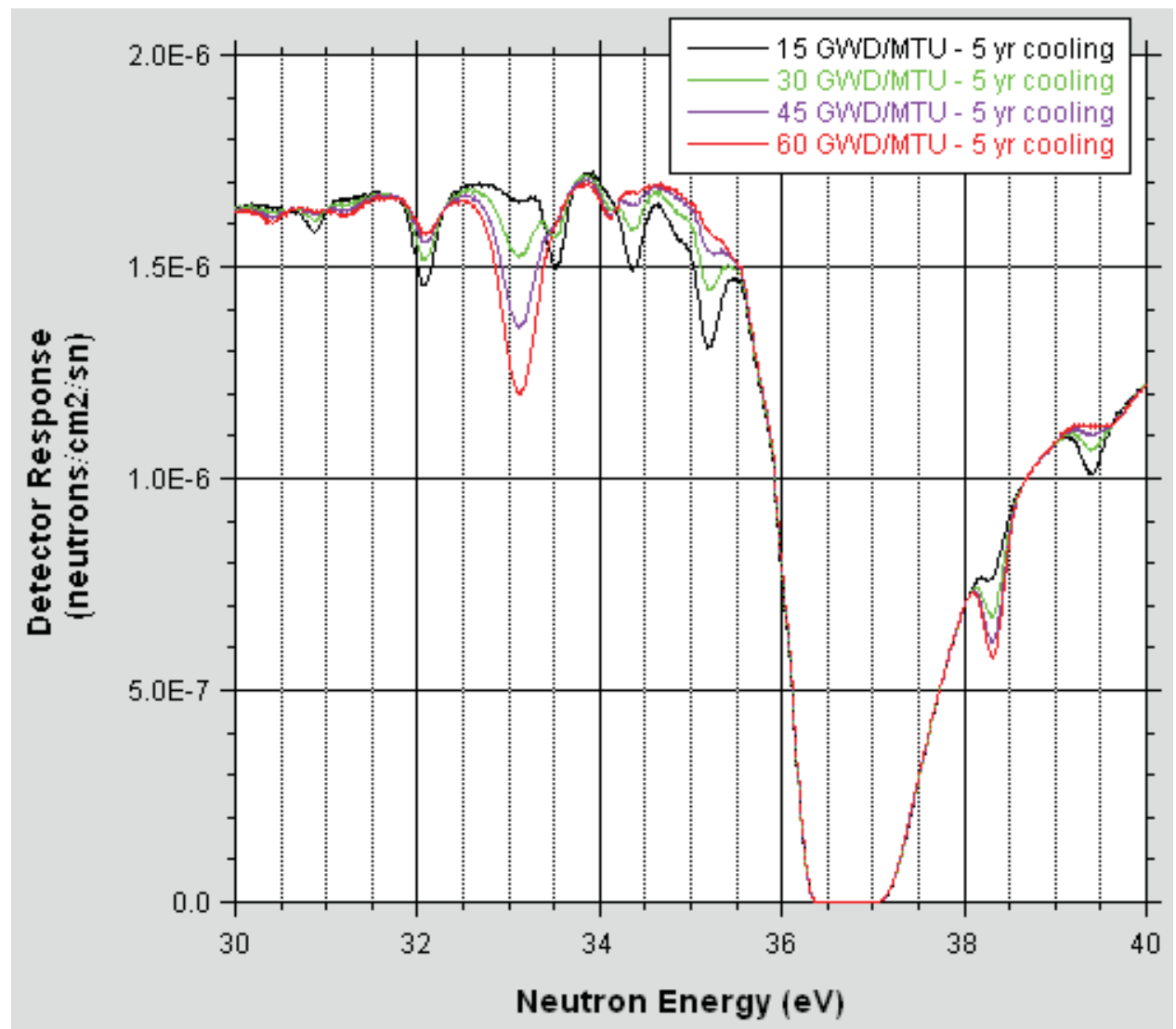

Figure 20 Transmission spectra as a function of burnup over the 30-40 eV energy range.

\subsection{Cooling Time}

This section compares calculated transmission spectra as a function of cooling time for two different fixed burnups. Spent-fuel composition data were again taken directly from the LANL 64 spent fuel library data for $3.0 \mathrm{wt} \%{ }^{235} \mathrm{U}$ initial enrichment, 30 and $60 \mathrm{GWD} / \mathrm{MTU}$ burnups, and 1, 5, 20, and 80-year cooling times. These transmission spectra are again for a single PWR fuel pin.

Initially, it was thought that there would be little or no difference between transmission spectra for different cooling times since the major actinides and fission products with resonance structure in the $0-40 \mathrm{eV}$ range were either stable or relatively long-lived. For example, the major fission products identified in the LANL spent-fuel 
compositions, including ${ }^{103} \mathrm{Rh},{ }^{131} \mathrm{Xe},{ }^{133} \mathrm{Cs},{ }^{145} \mathrm{Nd}$, and ${ }^{152} \mathrm{Sm}$, are all stable isotopes. The actinides ${ }^{235} \mathrm{U},{ }^{236} \mathrm{U},{ }^{238} \mathrm{U},{ }^{239} \mathrm{Pu},{ }^{240} \mathrm{Pu},{ }^{241} \mathrm{Pu}$, and ${ }^{242} \mathrm{Pu}$ are either stable or very long-lived relative to the longest cooling time of 80 years. An exception for the plutonium isotopes is ${ }^{241} \mathrm{Pu}$ (14.4 years), which was thought to be a possible indicator of long cooling times, although the single ${ }^{241} \mathrm{Pu}$ transmission depression at $13.4 \mathrm{eV}$ and 1-year decay is already relatively shallow (potentially difficult to assay but readily identifiable in a transmission spectrum). Therefore, no significant spectral differences were expected in the $0-40 \mathrm{eV}$ range as function of cooling.

Figure 21 shows the calculated transmission spectra as a function of cooling time for a fixed $30 \mathrm{GWD} / \mathrm{MTU}$ over the $0-40 \mathrm{eV}$ neutron energy range. Small variations in this figure hint at some sensitivity to cooling times due to isotopic buildup or decay but for the most part little variation is observed, as expected. One small but notable variation occurs for a resonance depression in the vicinity of $18 \mathrm{eV}$. This energy region is expanded in the next figure.

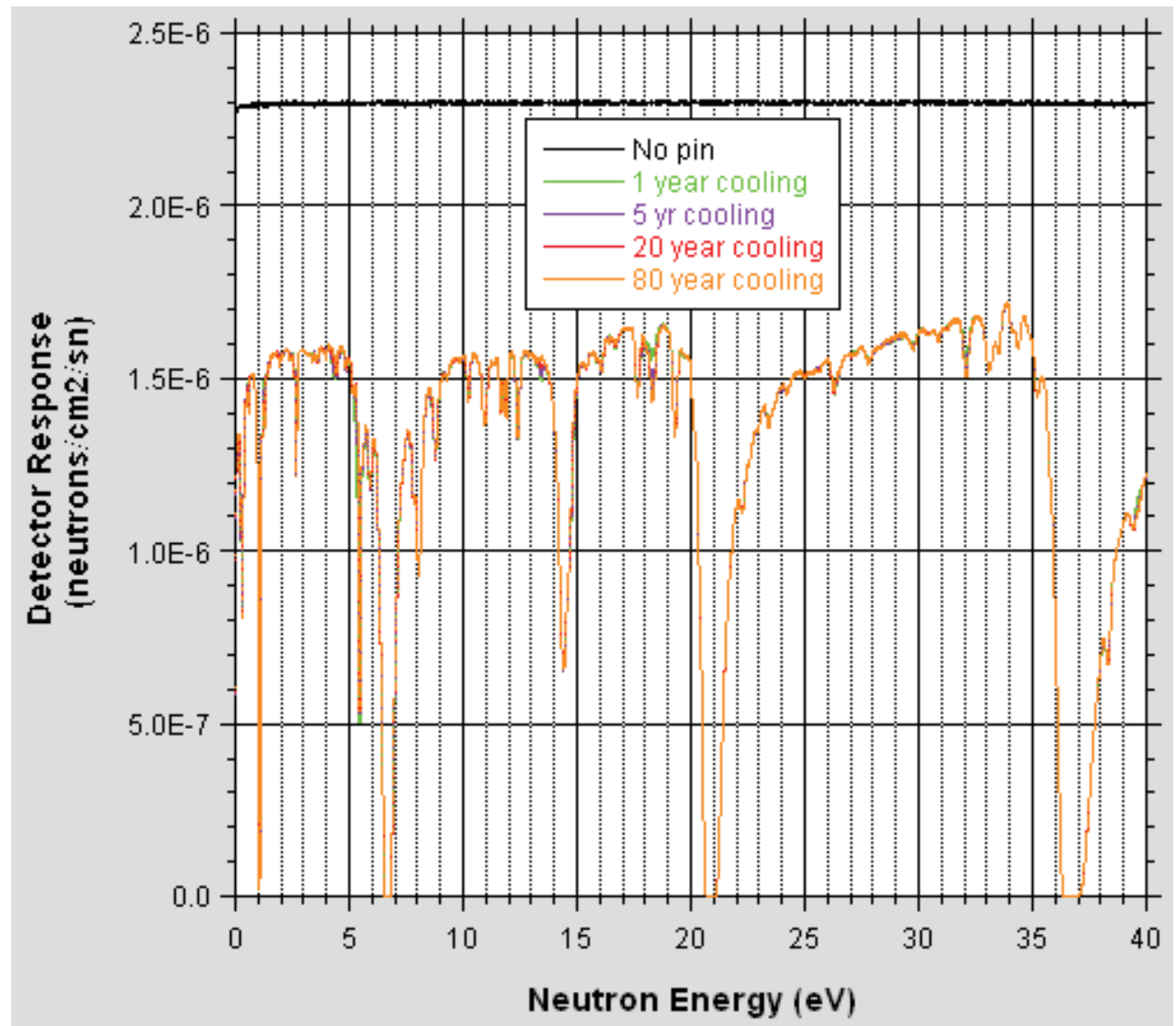

Figure 21 Transmission spectra as a function of cooling time for a fixed $\mathbf{3 0}$ GWD/MTU burnup (0-40 eV). 
Figure 22 shows the calculated transmission spectra as a function of cooling time for a fixed $30 \mathrm{GWD} / \mathrm{MTU}$ over the $0-20 \mathrm{eV}$ neutron energy range. The only two significant depressions are labeled, one for ${ }^{241} \mathrm{Pu}$ and another identified as the fission product ${ }^{147} \mathrm{Sm}$. Plutonium-241 decays into ${ }^{241} \mathrm{Am}$ with a 14.4 year half-life. The ${ }^{241} \mathrm{Pu}$ depression in the transmitted neutron spectrum eventually disappears after cooling times greater than 40 years. The ${ }^{147} \mathrm{Sm}$ depression, on the other hand, increases in magnitude with cooling time apparently due to an increase in the stable ${ }^{147} \mathrm{Sm}$ concentration over in time. The next figure magnifies the energy region around the $18 \mathrm{eV}$ resonance.

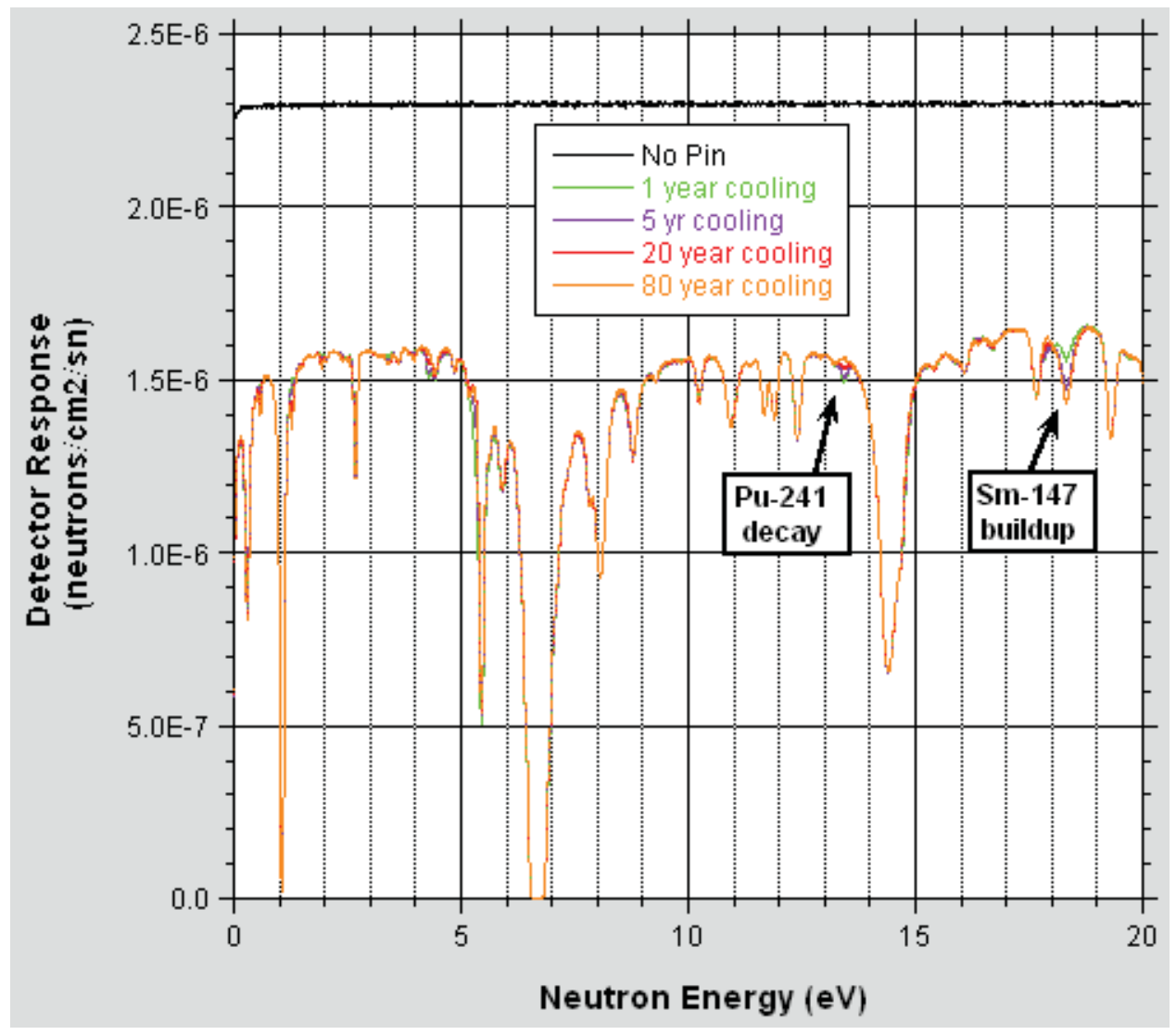

Figure 22 Transmission spectra as a function of cooling time for a fixed $\mathbf{3 0}$ GWD/MTU burnup (0-20 eV). 
Figure 23 shows the ${ }^{147} \mathrm{Sm}$ resonance depression at $18.25 \mathrm{eV}$ over the $15-20 \mathrm{eV}$ energy range. This resonance depression increases in magnitude with cooling time and may be a significant, easily identifiable NRTA marker for cooling time, in particular cooling times greater than 1-year and less than 15-years.

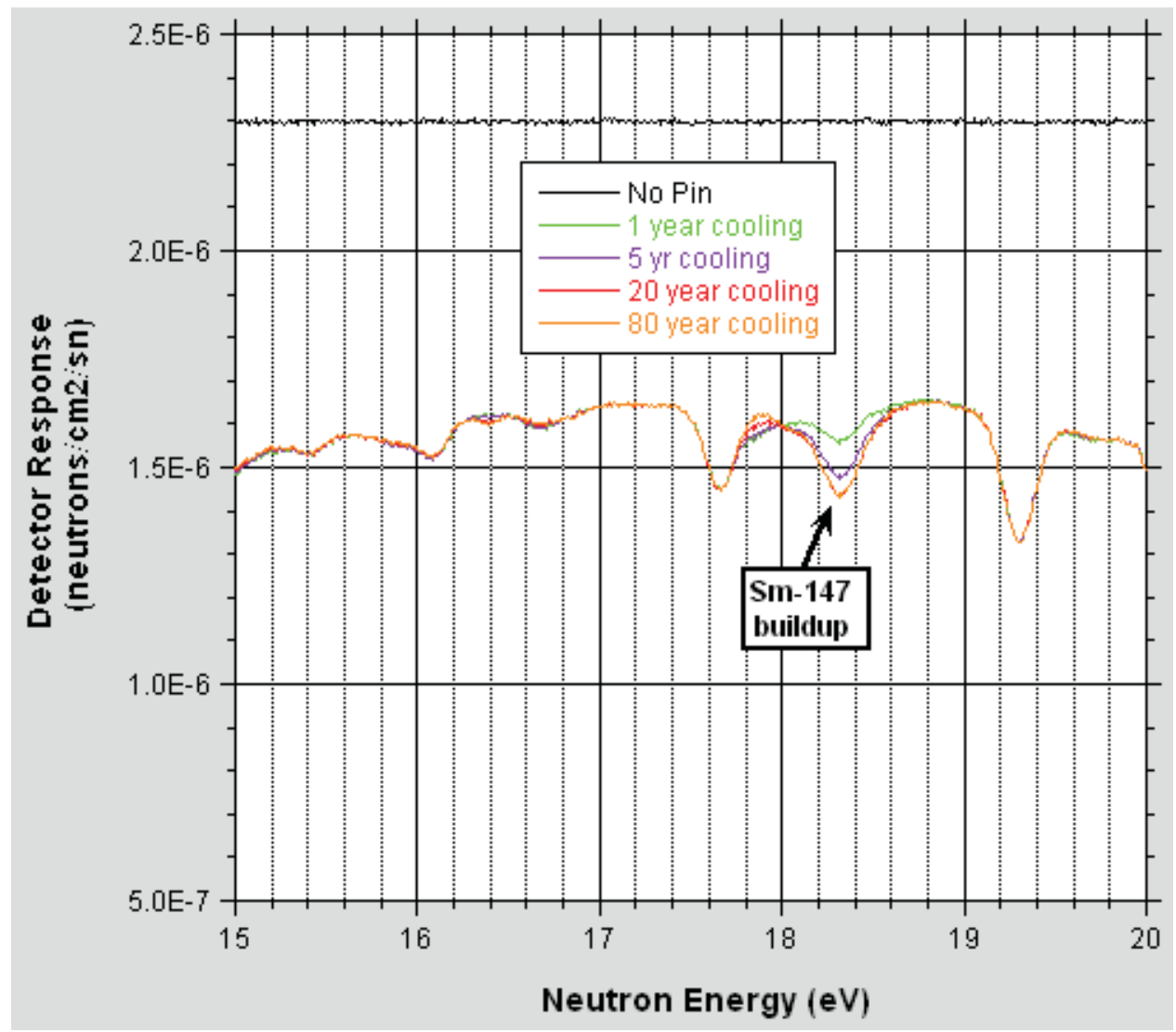

Figure 23 Transmission spectra as a function of cooling time for a fixed $\mathbf{3 0}$ GWD/MTU burnup (15-20 eV). 
Figure 24 shows the transmission spectra for the 60 GWD/MTU burnup case but only over the range of $15-20 \mathrm{eV}$, in order to feature the effect of ${ }^{147} \mathrm{Sm}$ buildup with cooling time. The ${ }^{147} \mathrm{Sm}$ depression is only slightly greater in magnitude (deeper) for the $60 \mathrm{GWD} / \mathrm{MTU}$ burnup (Figure 24) versus the $30 \mathrm{GWD} / \mathrm{MTU}$ case (Figure 23).

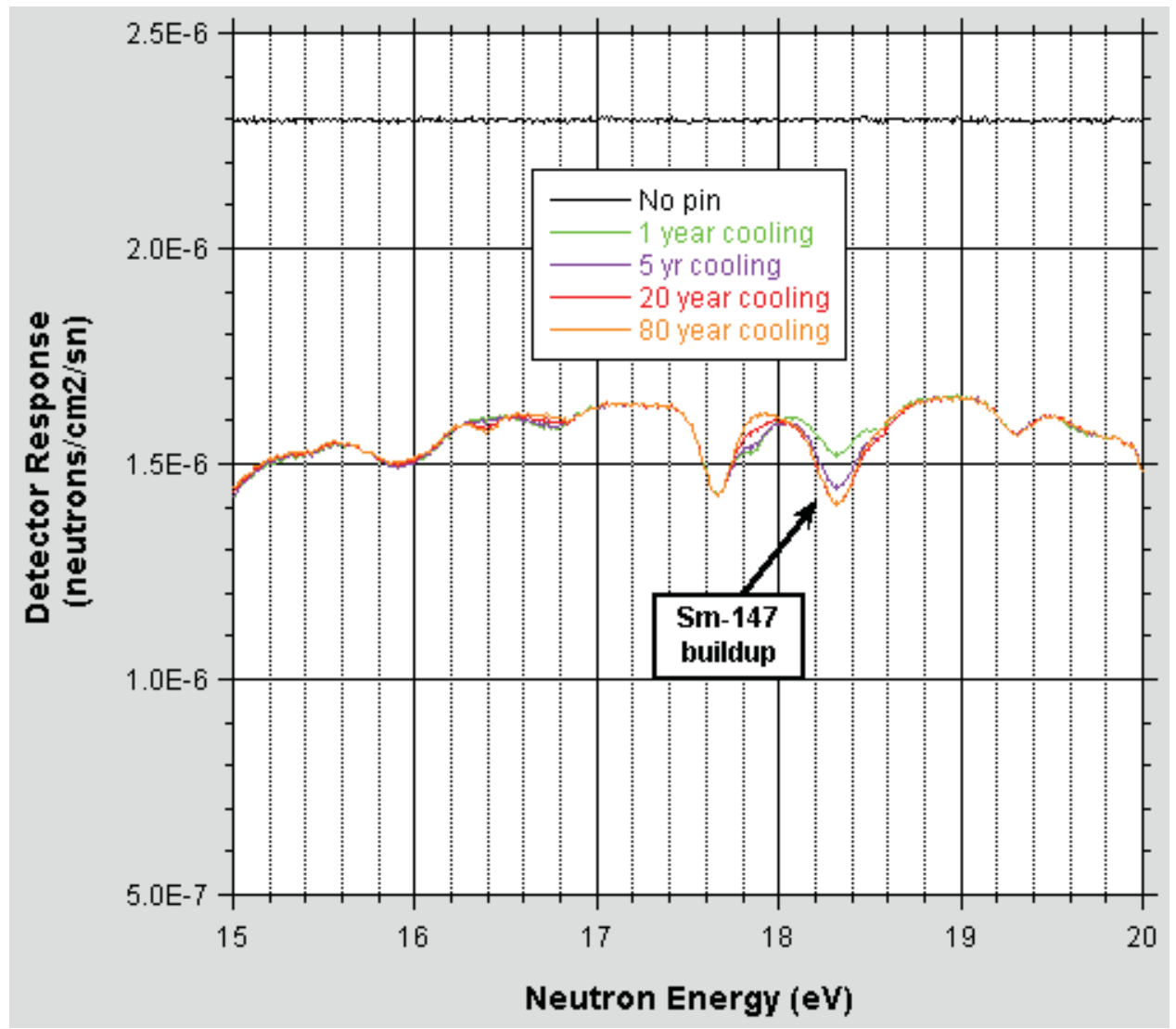

Figure 24 Transmission spectra as a function of cooling time for a fixed 60 GWD/MTU burnup (15-20 eV).

Samarium-147 is a stable fission product at the end of the $\mathrm{A}=147$ beta-decay chain. This particular chain has a significant $2.25 \%$ fission yield from ${ }^{235} \mathrm{U}$ thermalneutron fission. The parent radionuclide above ${ }^{147} \mathrm{Sm}$ in the beta decay chain is ${ }^{147} \mathrm{Pm}$. Promethium-147 has a 2.6234 year half-life, which allows for an exponential buildup of ${ }^{147} \mathrm{Sm}$ to a maximum concentration after approximately 13 years ( 5 half-lives of $\left.{ }^{147} \mathrm{Pm}\right)$. In addition to the $\mathrm{A}=147$ beta-decay chain feeding ${ }^{147} \mathrm{Sm}$ buildup, ${ }^{147} \mathrm{Sm}$ also has a direct fission product yield. The direct yield is small, however, and the buildup ${ }^{147} \mathrm{Sm}$ can be attributed almost entirely to ${ }^{147} \mathrm{Pm}$ decay. Hence, ${ }^{147} \mathrm{Sm}$ could possibly be used to estimate spent-fuel cooling times, at least for cooling times between 0-15 years.

The following is a proposed method in which the cooling time might be estimated using both an NRTA assay measurement of ${ }^{147} \mathrm{Sm}$ and our high-fidelity burnup calculations. First, the NRTA technique would be used to determine, in one of the corner pins of the PWR assembly (single pin assay), the mass of ${ }^{147} \mathrm{Sm}$ and the pin burnup (GWD/MTU). Then, in conjunction with the high-fidelity burnup calculation and the assembly in-reactor history, the mass of ${ }^{147} \mathrm{Sm}$ and ${ }^{147} \mathrm{Pm}$ at the end-of-life (EOL) with no 
cooling time could be estimated for the pin. The difference between the measured ${ }^{147} \mathrm{Sm}$ mass and EOL calculated mass would be equivalent to the amount of ${ }^{147} \mathrm{Pm}$ that has decayed from EOL to the measurement date. The assembly cooling time can be estimated using Eq. 2,

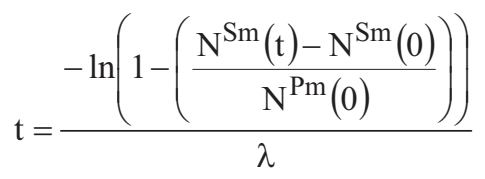

where:

$\mathrm{t}=$ cooling time, or time between assembly EOL and the NRTA measurement,

$\mathrm{N}^{\mathrm{Sm}}(\mathrm{t})=$ number of atoms of ${ }^{147} \mathrm{Sm}$ determined from the NRTA measurement at time $\mathrm{t}$,

$\mathrm{N}^{\mathrm{Sm}}(0)=$ number of atoms of ${ }^{147} \mathrm{Sm}$ determined from the burnup calculation at EOL, when $\mathrm{t}=0$,

$\mathrm{N}^{\mathrm{Pm}}(0)=$ number of atoms of ${ }^{147} \mathrm{Pm}$ determined from the burnup calculation at EOL when $\mathrm{t}=0$, and

$\lambda=$ decay constant for ${ }^{147} \mathrm{Pm}\left(0.264217 \mathrm{yr}^{-1}\right)$.

If estimating cooling time is desired as part of the NGSI an NRTA measurement of the ${ }^{147} \mathrm{Sm}$ resonance depression at $18.25 \mathrm{eV}$ could provide such an estimate. This resonance depression falls within the $0-20 \mathrm{eV}$ energy range proposed for NRTA transmission measurements.

\subsection{Cylindrical Beam Diameter}

The irradiating neutron transmission beam can be collimated into a variety of geometrical shapes and sizes. The choice of beam shape and size will be influenced by a variety of factors, including fuel-pellet diameter, pellet spatial resolution, assembly pin pitch, and size and spatial resolution of the detectors. Available counting time, accelerator system neutron-emission rate, flight-tube diameter, and desired volume of assembly to be assayed are related factors. Therefore, selection of a beam geometry and size will evolve as the definition of the NRTA system concept and components synergistically evolve.

This section focuses on calculated transmission spectral results for a cylindrical beam with a variable diameter. The beam was directed perpendicular to and centered on the vertical axis of a single fuel pin. The beam diameter was varied from zero to $1.0 \mathrm{~cm}$, where a zero-cm beam diameter corresponds to a hypothetical pencil-beam source of neutrons directed at the pin through the center, thickest part of the fuel pin. The beam diameter was progressively increased from 0.0 to $1.0 \mathrm{~cm}$ in increments of $0.2 \mathrm{~cm}$. At a beam diameter of $0.8 \mathrm{~cm}$ the beam encompasses virtually the entire fuel pellet $(0.82 \mathrm{~cm})$. At $1.0 \mathrm{~cm}$; the beam diameter exceeds both the $0.82 \mathrm{~cm} \mathrm{UO}_{2}$ pellet diameter and the 0.95 $\mathrm{cm}$ pin diameter as well. In this case, some transmission neutrons will pass by or around the $\mathrm{UO}_{2}$ unattenuated and yet will still be counted by the detector. This will have the effect of increasing the number of counts across the energy spectrum $(0-40 \mathrm{eV})$, resulting in an upward magnitude shift of the entire transmission spectrum. It is not clear yet how 
this affects the assay analysis, or whether it can be compensated through normalization techniques or eliminated with detectors that can spatially resolve a fuel pellet.

Figure 25 shows the MCNP-calculated transmitted signal as a function of the cylindrical beam diameter. The MCNP-flux tally detector is a $10 \times 10 \mathrm{~cm}$ detector, much larger than the $\mathrm{UO}_{2}$ fuel pellet diameter.

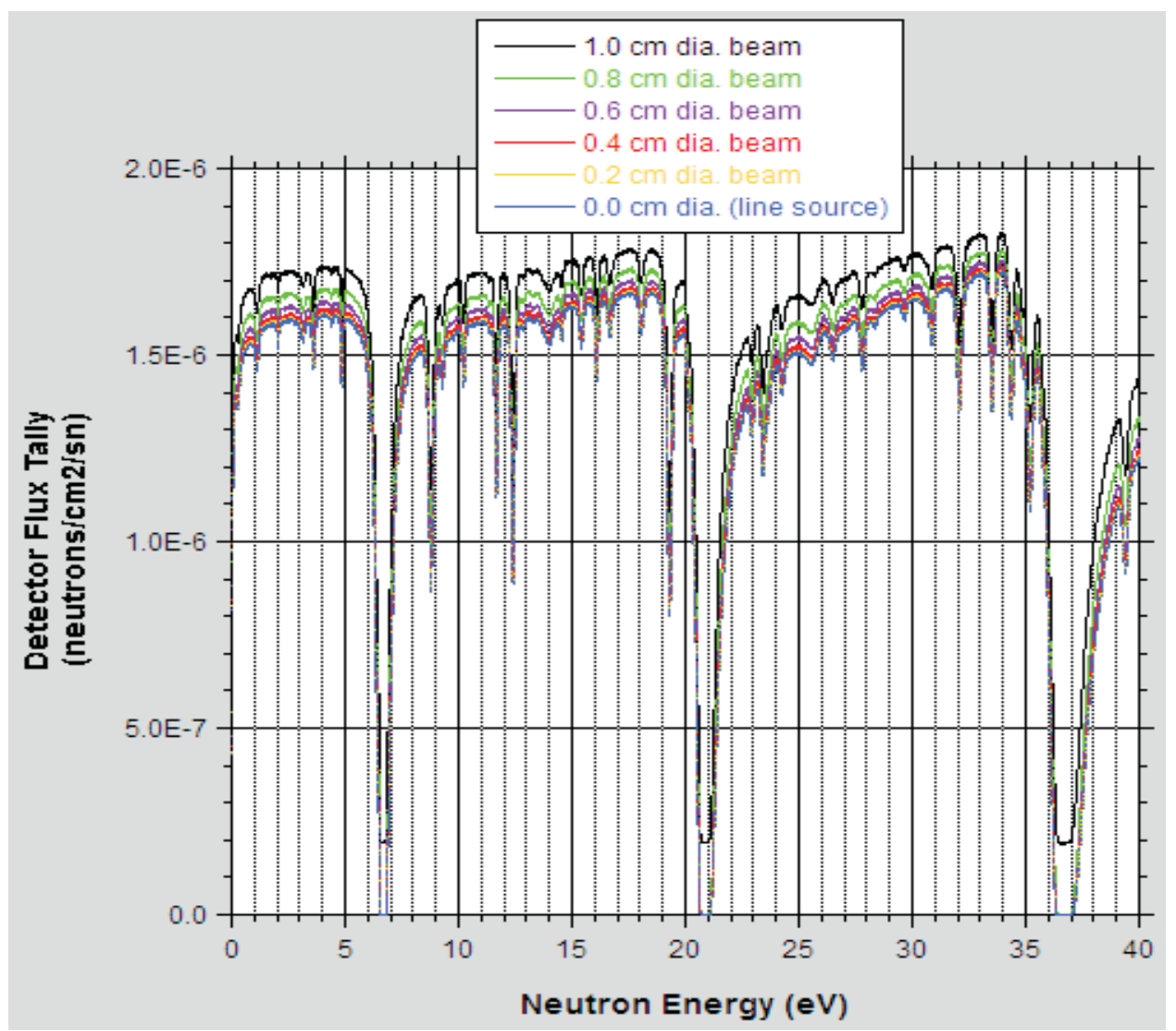

Figure 25 MCNP-calculated transmitted signal as a function of cylindrical beam diameter.

In Figure 25 the MCNP-calculated transmission spectra, or detector flux tallies, decrease in magnitude (per source neutron) with each decrease in the beam diameter. The beam must continually traverse an effectively thicker section of the $\mathrm{UO}_{2}$ pellet and the result is an increase in beam attenuation. Eventually, the beam diameter reaches zero $\mathrm{cm}$, or a hypothetical pencil-beam of neutrons traversing the thickest part of the $\mathrm{UO}_{2}$ pellet, directly through its center, thus maximizing the neutron beam attenuation (or neutron beam out-scatter). For the case in which the neutron beam diameter $(1.0 \mathrm{~cm})$ is larger than the pellet diameter $(0.82 \mathrm{~cm})$ the detected signal per source neutron is strongest; neutrons can stream without attenuation through the clad or through lattice air spaces between the pins, thus maximizing the transmitted signal.

The study shows the transmission signal strength through the $\mathrm{UO}_{2}$ pellet is affected by the beam diameter and pellet curvature. The next study uses a pencil-beam source of neutrons (zero-cm diameter) to sweep transversely across the $\mathrm{UO}_{2}$ fuel pellet from pellet center to pin periphery. Transmission signal strength increases closer to the 
pellet periphery, raising the prospect for deeper assembly penetration and transmission assays for up to 12 pins. Also, it can be envisioned that multiple zero-cm diameter beams could be stacked vertically to create a planar- or fan-shaped beam. In practice this fanshaped beam could be achieved by using a narrow-slit collimator with the long side of the rectangular slit oriented parallel to the pin's vertical axis.

\subsection{Fan-shaped Beam}

The fan-shaped beam mentioned in the Section 4.5 has also been proposed in the literature, for a case in which the sample is pulled across the neutron beam.[2] For application to a PWR fuel pin the fan-plane would be oriented in the vertical direction (parallel to the fuel pin axis) with a fan-thickness (or narrow-slit thickness of the collimator) on the order of $0.1-0.2 \mathrm{~cm}$ in order to spatially resolve a fuel pellet across its diameter $(0.82 \mathrm{~cm})$. The slow and tedious problem remains to move the assembly across the beam at each spatial increment and take a count.

An alternative and perhaps more practical approach would be to use a largeeffective-area detector array capable of resolving transmission data in two dimensions. A gas electron multiplier operating with a multi-strip detector might be suitable for this measurement. Another approach might be to use an array of ${ }^{6} \mathrm{Li}$-doped, small-diameter scintillator fibers facing the assembly. The pixel-dimensions for a 2-D detector would be chosen based on the pellet spatial resolution desired for the measurement and the geometry of the fan-beam, together with other system parameters including the counting time, accelerator neutron output, and scan coverage of the assembly (number of scans needed to assay an entire assembly). This alternative approach eliminates the need for a narrow-slit beam and allows for a much wider irradiating neutron beam. The wider beam would be comparable in width to the width of the detector array; a width encompassing perhaps two or three fuel pins. This alternative approach would also eliminate the need to move the assembly across the beam in painfully small increments and, therefore, allow for scanning a much larger section of the assembly for each count period.

\subsection{Directed Point Source - Pencil Beam Irradiation}

This section considers a pencil beam of neutrons aimed at a single fresh fuel pin (3.2 $\mathrm{wt} \%{ }^{235} \mathrm{U}$ enrichment). The beam is directed perpendicular to the fuel pin axis and is moved transversely across the fuel pin. The line of neutrons is initially pointed through the pin axis (thickest part of pellet) then moved transversely or laterally along the y-axis through increasing thinner sections, or chords, of the pellet (see Figure 26). The transmitted signal is expected to get progressively stronger (less out-scatter and consequently shallower transmission depressions) as the source sweeps toward the pellet periphery and the $\mathrm{UO}_{2}$ chord lengths get shorter. Beyond the fuel pellet radius $(>0.4095$ $\mathrm{cm}$ ), the neutron beam is transmitted freely with virtually no attenuation through the pellet-clad gap and the clad. 


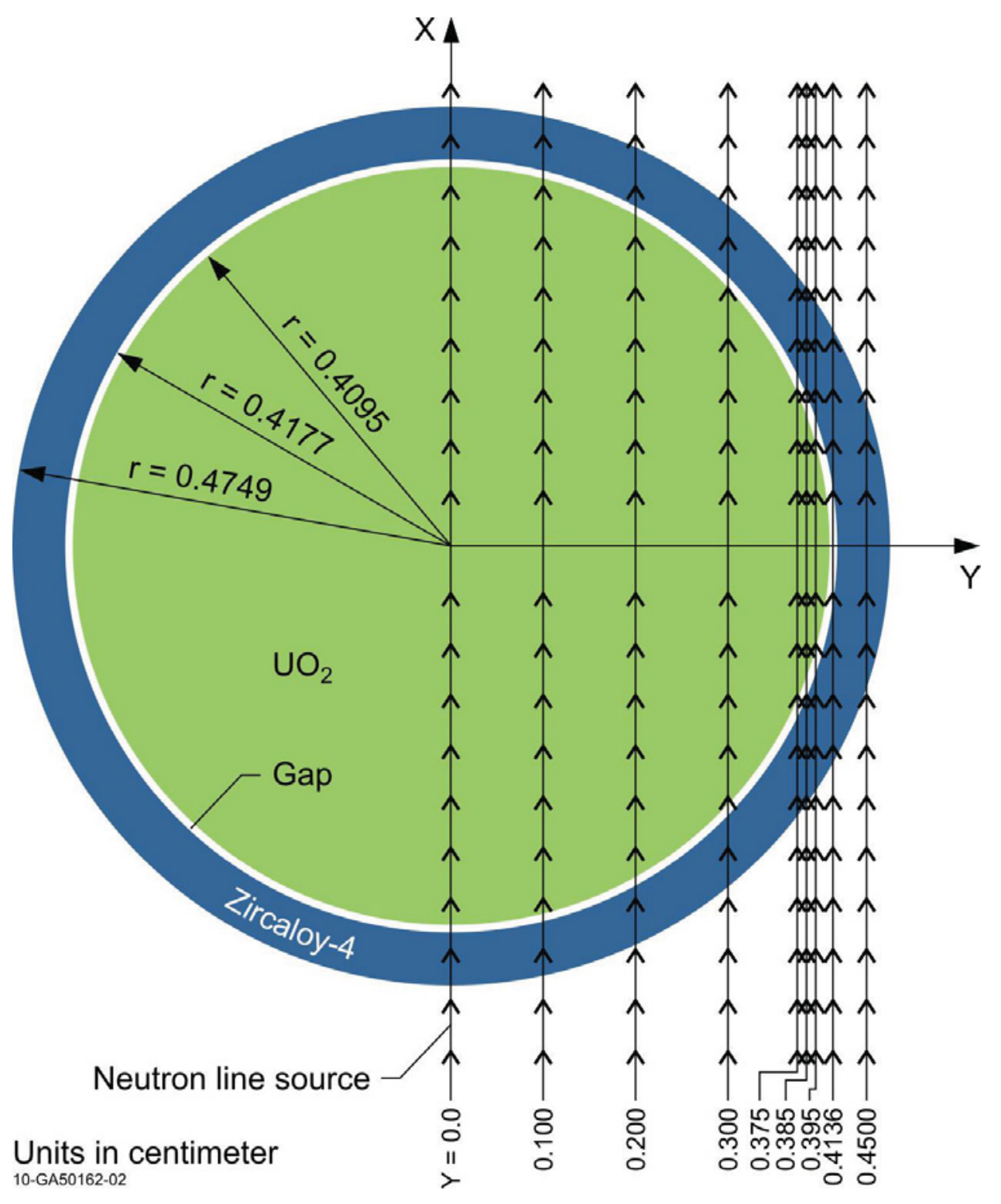

Figure 26 Geometry for the directed point source and fuel pin.

Figure 27 shows the calculated neutron resonance transmission signal for seven different chord lengths, corresponding to the specific y-axis locations in Figure 26: $\mathrm{y}=$ $0.0,0.1,0.2,0.3,0.375,0.385,0.395,0.4136$, and $0.45 \mathrm{~cm}$. For this particular study, it was assumed that the $\mathrm{UO}_{2}$ pellet radius was 0.4095 , and the inner and outer clad radii were 0.4177 and $0.4749 \mathrm{~cm}$. This fresh fuel pin has a gap between the pellet and the clad $(\mathrm{r}=0.4095$ to $0.4177 \mathrm{~cm})$, such that at $\mathrm{y}=0.4136 \mathrm{~cm}$ the neutron pencil beam passes through the gap. At $\mathrm{y}=0.0 \mathrm{~cm}$ the neutrons pass through the thickest chord of $\mathrm{UO}_{2}$ and at $\mathrm{y}=0.395 \mathrm{~cm}$ the neutrons pass through the thinnest $\mathrm{UO}_{2}$ chord. 


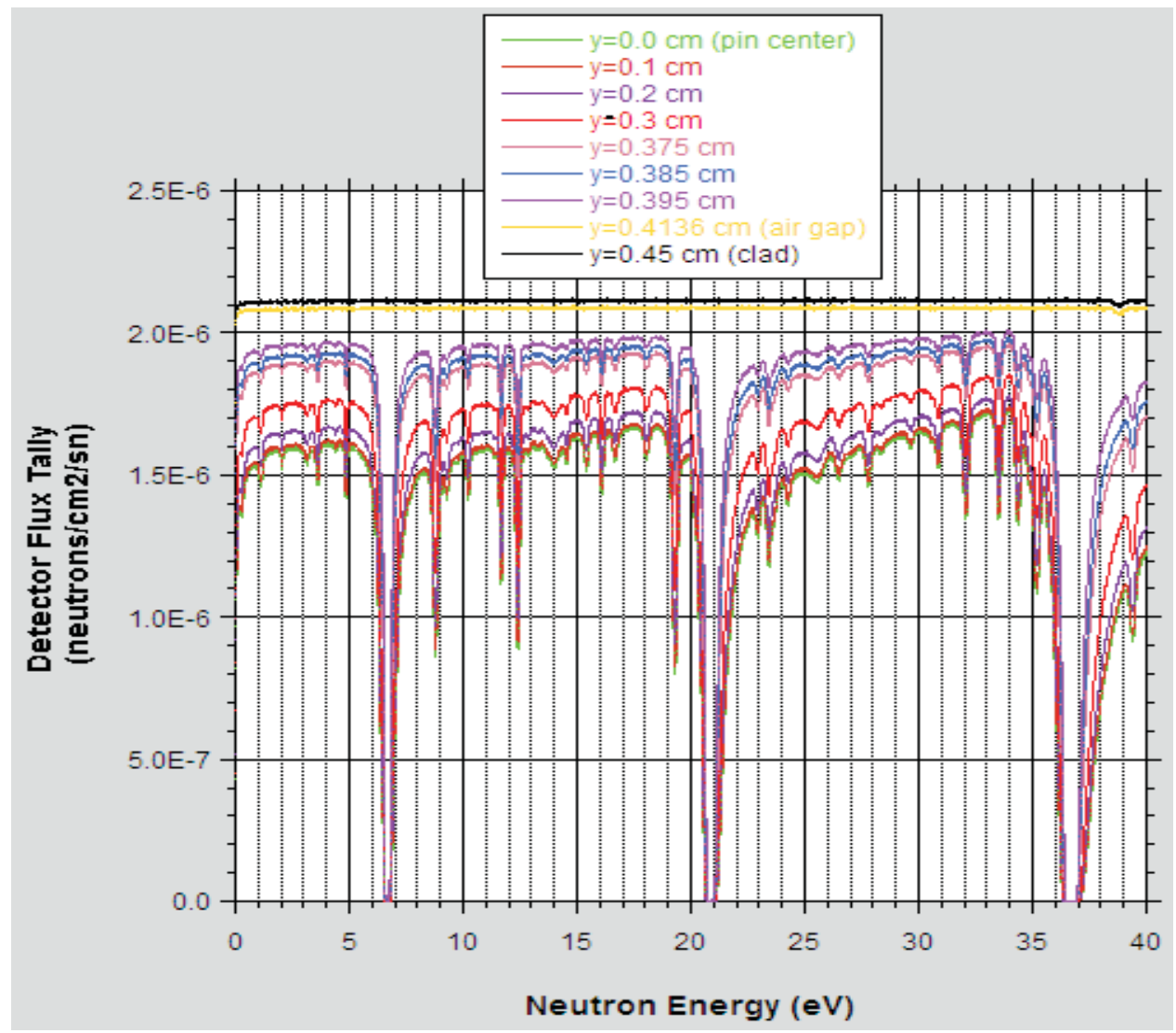

Figure 27 Neutron transmission spectra for a directed point source across a $\mathrm{UO}_{2}$ pellet/pin over the $0-40 \mathrm{eV}$ energy range.

Figure 27 shows substantial magnitude increase in the transmission signal as the beam moves along the $\mathrm{y}$-axis from $\mathrm{y}=0.0$ to $\mathrm{y}=0.395 \mathrm{~cm}$. The neutron out-scatter decreases with decreasing $\mathrm{UO}_{2}$ chord thickness. This effect may be useful in the quest to achieve greater penetrability into a PWR assembly, which will translate into being able to include more fuel pins in a given transmission assay signal. 
Figure 28 is an expanded view of the transmission spectrum in Figure 27. Shown here is the $18-20 \mathrm{eV}$ energy range, encompassing the $19.3 \mathrm{eV}^{235} \mathrm{U}$ resonance depression. As the beam moves along the $\mathrm{y}$-axis from $\mathrm{y}=0.0$ to $0.395 \mathrm{~cm}$ and traverses shorter $\mathrm{UO}_{2}$ chords, the resonance depression increases in magnitude (less out-scatter). The reduction in the resonance depression, particularly near the pellet periphery, appears to be a measureable effect and, consequently, could allow the NRTA technique to assay more fuel pins in a row, perhaps up to 12 pins.

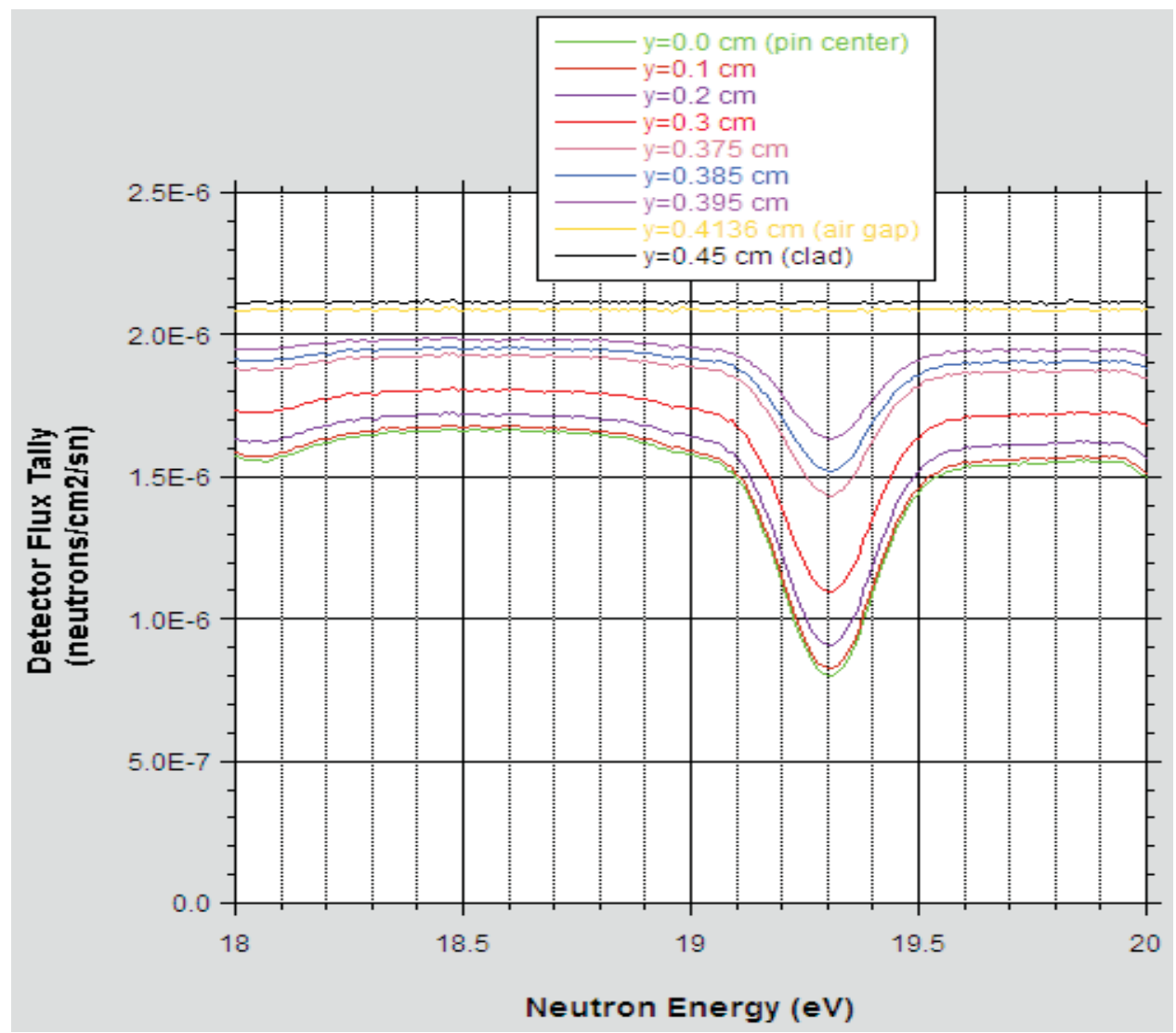

Figure 28 Neutron transmission spectra for a directed point source across a $\mathrm{UO}_{2}$ pellet/pin over just the $18-20 \mathrm{eV}$ energy range.

\subsection{Multiple Fuel Pins}

This section presents calculated transmission spectra through multiple fuel pins. For the $17 \times 17$ PWR fuel assembly (Figure 4) there are rows of fuel pins with up to 17 pins in a row. Based on the preliminary MCNP-calculated transmission spectrum herein, a 17-pin row may not permit accurate assays with the NRTA technique; however, 8-12 pins may be possible. As mentioned above, if 12 pins can be assayed, then every pin in the $17 \times 17$ assembly can be part of one or more NRTA row assays. A twelve pin assay may be more feasible if a narrow fan-shaped beam were swept across a 12-pin row starting at one edge of the clad of the first pin and sweeping the beam across the pin to the other side. 
Neutron transmission is increased for shorter $\mathrm{UO}_{2}$ chord sections. The fan-shaped sweep of course would rely on a straight line of pins (no significant pin bowing or misalignment) and a narrow fan-shaped beam thickness. The MCNP calculations assume perfect pin alignment and an infinitely thin fan-shaped beam (directed point source). These are possible, hence, the calculated results give a good indication of what is theoretically possible, if not completely obtainable, in practice.

Figure 29 shows the neutron transmission spectra through multiple spent fuel pins of identical dimensions and material compositions, perfectly aligned in a row. The neutron beam is assumed to be a directed point source of neutrons through the geometric center of the fuel pins at $\mathrm{y}=0.0 \mathrm{~cm}$ (thickest chord through the $\mathrm{UO}_{2}$ pellet, see Figure 26) and perpendicular to the pin axis (vertical z-direction). The $\mathrm{UO}_{2}$ spent-fuel material composition is an arbitrarily selected pin composition (m704) from the LANL library with $30 \mathrm{GWD} / \mathrm{MTU}$ burnup, 1-year cooling time, and an initial $3.0 \mathrm{wt} \%{ }^{235} \mathrm{U}$ enrichment.

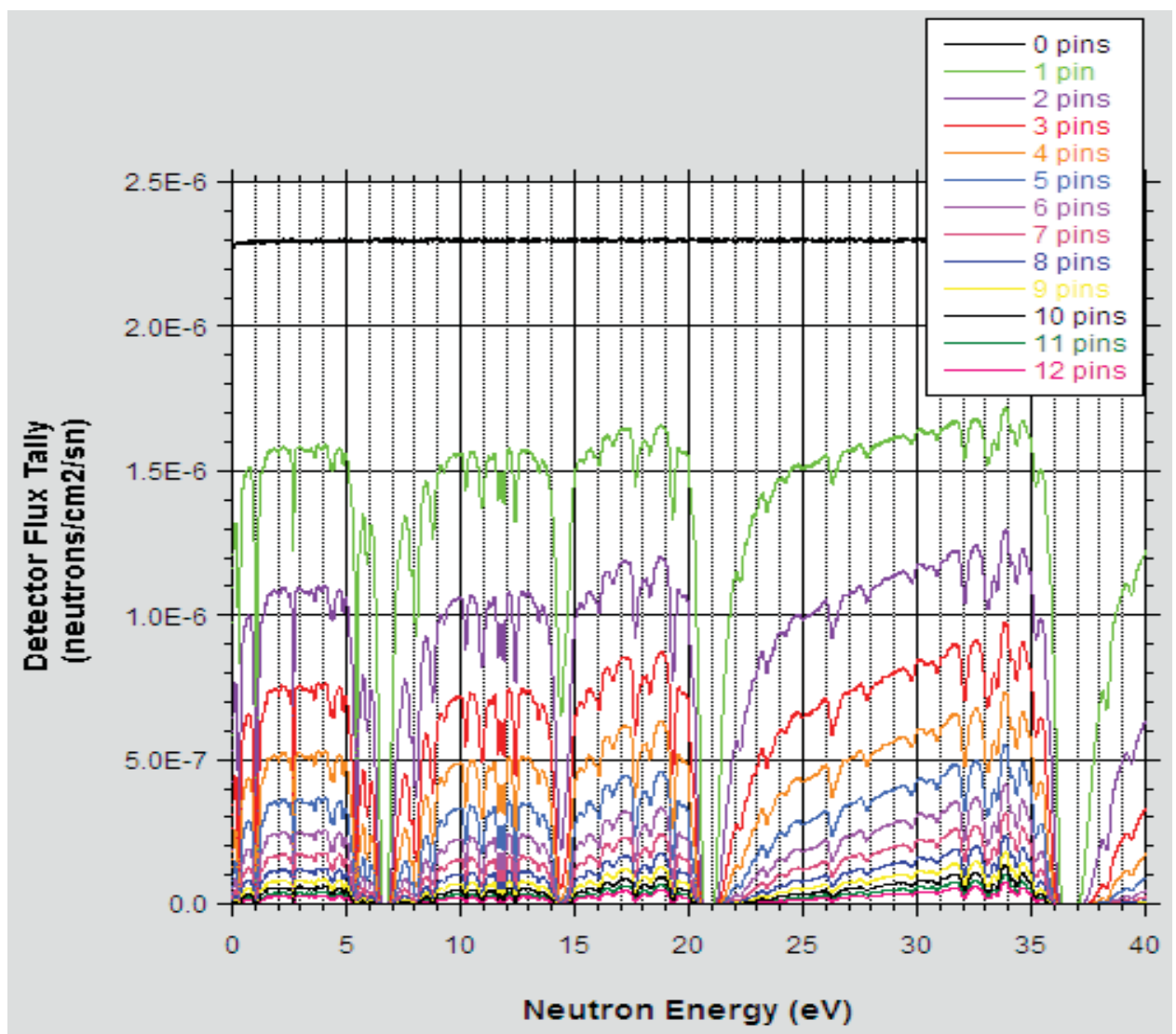

Figure 29 Neutron resonance transmission spectra through multiple spent fuel pins $(0-40 \mathrm{eV})$ at $\mathrm{y}=0.0 \mathrm{~cm}$.

Figure 29 clearly shows the transmission signal attenuation as a function of the number of pins in a row. After approximately 9-10 pins, the difference between transmission spectra in the non-resonance regions is small. Eight or nine pins, assayed through the center chord of each pin, may be the limit for the NRTA technique. For an 8pin maximum, $64 \%$ of the fuel pins in an assembly can be part of an integral transmission assay measurement (see Figure 4: Nine diagonal scans per quadrant with 8 pins or less starting at the lower right corner pin and moving upward through the assembly). As 
shown below, a pencil-beam neutron source scanning transversely across the fuel pin can produce differentiated transmission signals for which a 12-pin scan may be possible, Oen hundred percent of the fuel pins in an assembly could then be part of an integral transmission assay measurement. The neutron-beam geometry would need to be collimated into a narrow fan-shaped beam with the fan plane parallel to the pin axis. For interest, Figure 30 through Figure 32 provide expanded views of Figure 29 in order to show more detail of the transmitted signal over the $0-40 \mathrm{eV}$ energy range.

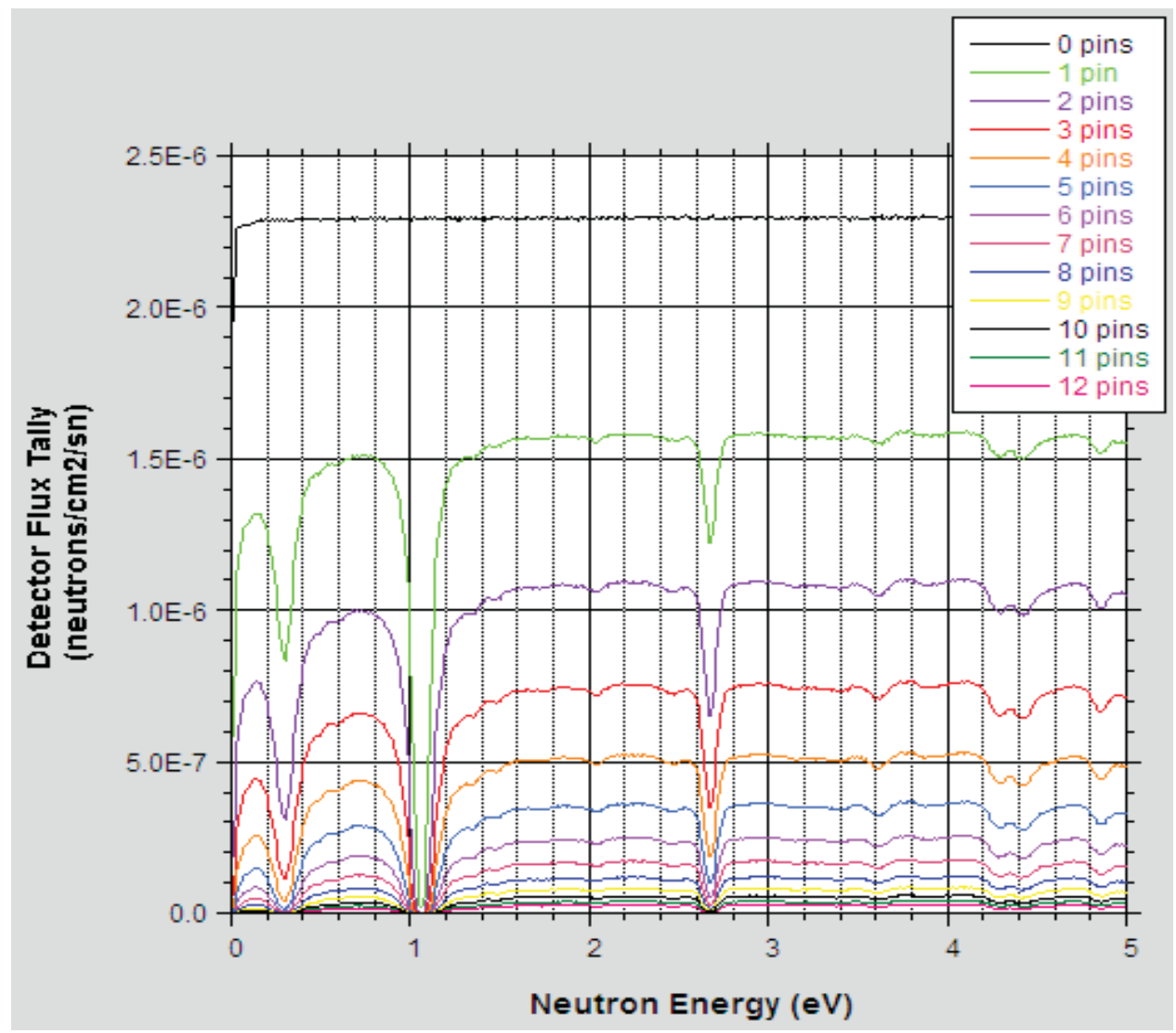

Figure 30 Neutron resonance transmission spectra through multiple spent fuel pins $(0-5 \mathrm{eV})$ at $\mathbf{y}=0.0 \mathrm{~cm}$. 


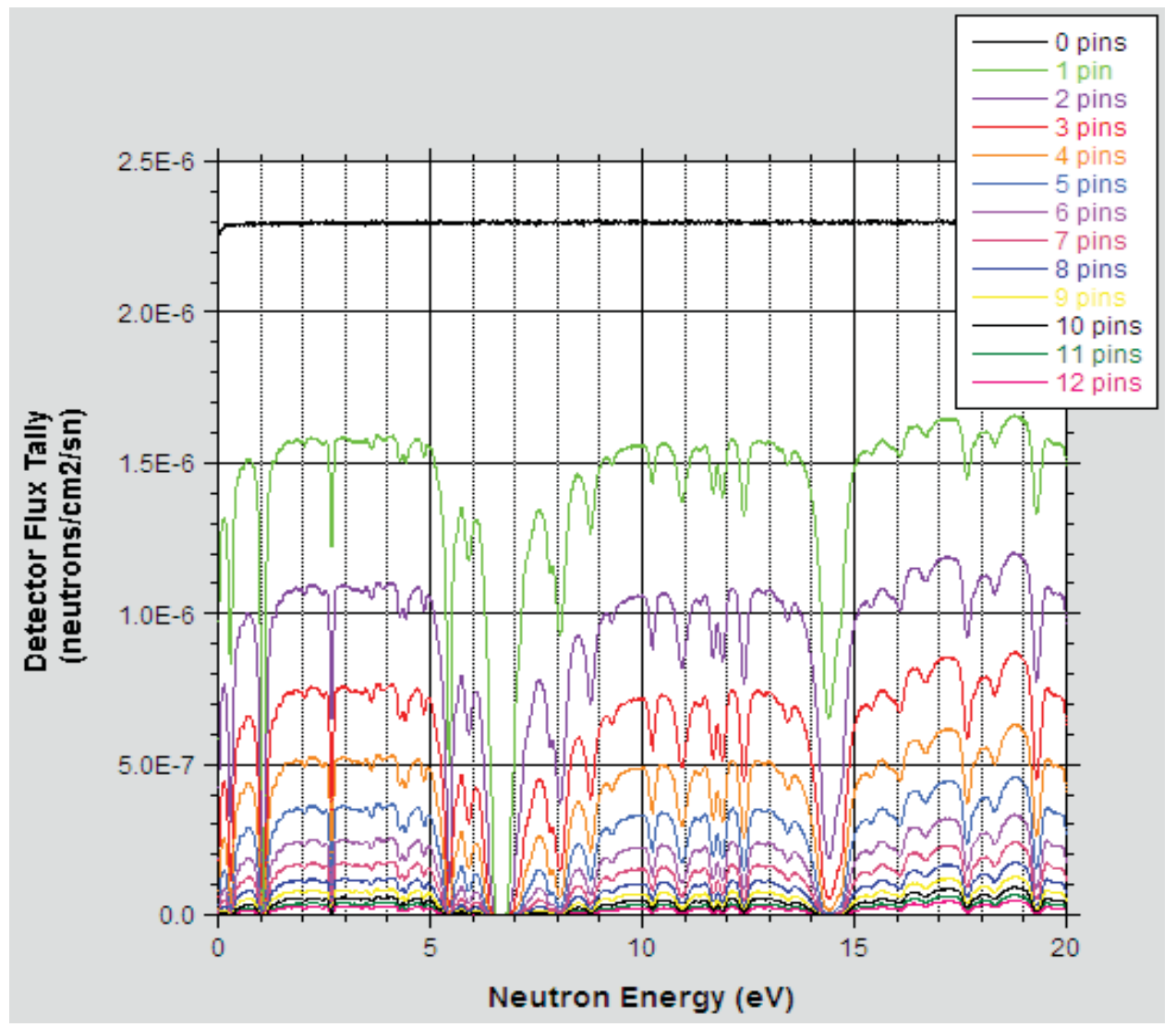

Figure 31 Neutron resonance transmission spectra through multiple spent fuel pins $(0-20 \mathrm{eV})$ at $\mathbf{y}=0.0 \mathrm{~cm}$. 


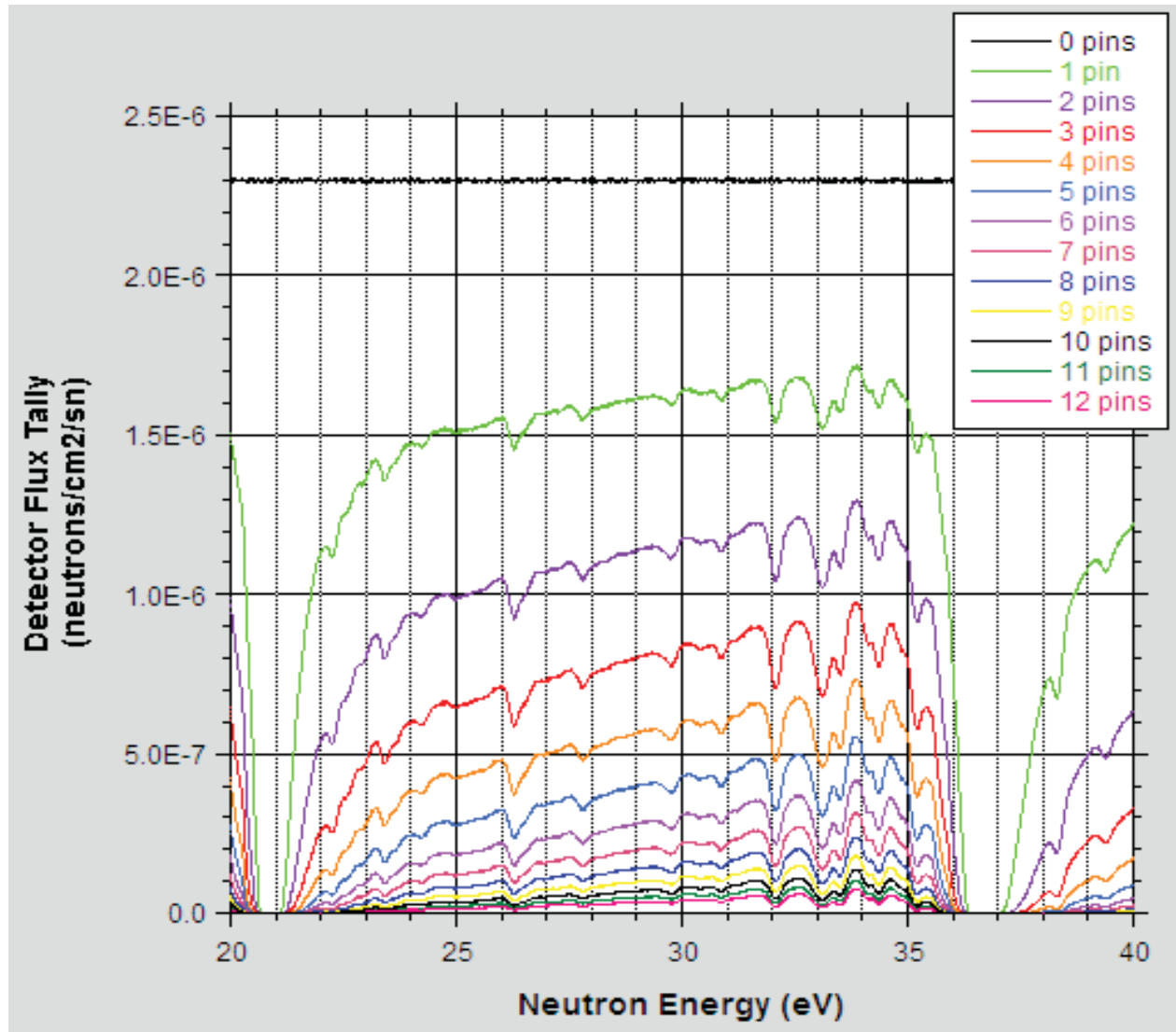

Figure 32 Neutron resonance transmission spectra through multiple spent fuel pins $(20-40 \mathrm{eV})$ at $\mathrm{y}=0.0 \mathrm{~cm}$.

In Figure 29 through Figure 32, transmission through the very first fuel pin results in a $30-35 \%$ reduction in the signal. With the addition of more pins the transmission signal continues to decrease in magnitude. After 9 pins the differential between signals is so small that a measureable difference would probably not be possible. It would be desirable to make the attenuation, or difference between signals, more uniform in magnitude for each additional pin added to a row. One way to reduce the sharp exponential decrease in the signal magnitude is to move the pencil-beam source off-axis, toward the pin periphery or clad (or to use a 2-dimensional neutron detector). For example, if the point source is moved close to the clad at the $y=0.375 \mathrm{~cm}$ location, then the chord thickness per pin and the total $\mathrm{UO}_{2}$ thickness traversed by the beam is reduced.

Figure 33 shows calculated transmission signals for 0-12 pins in a row but here specifically for the off-axis point source located at $y=0.375 \mathrm{~cm}$. Figure 34 through Figure 36 are identical to Figure 33, but expanded to show specific energy ranges between $0-40 \mathrm{eV}$. 


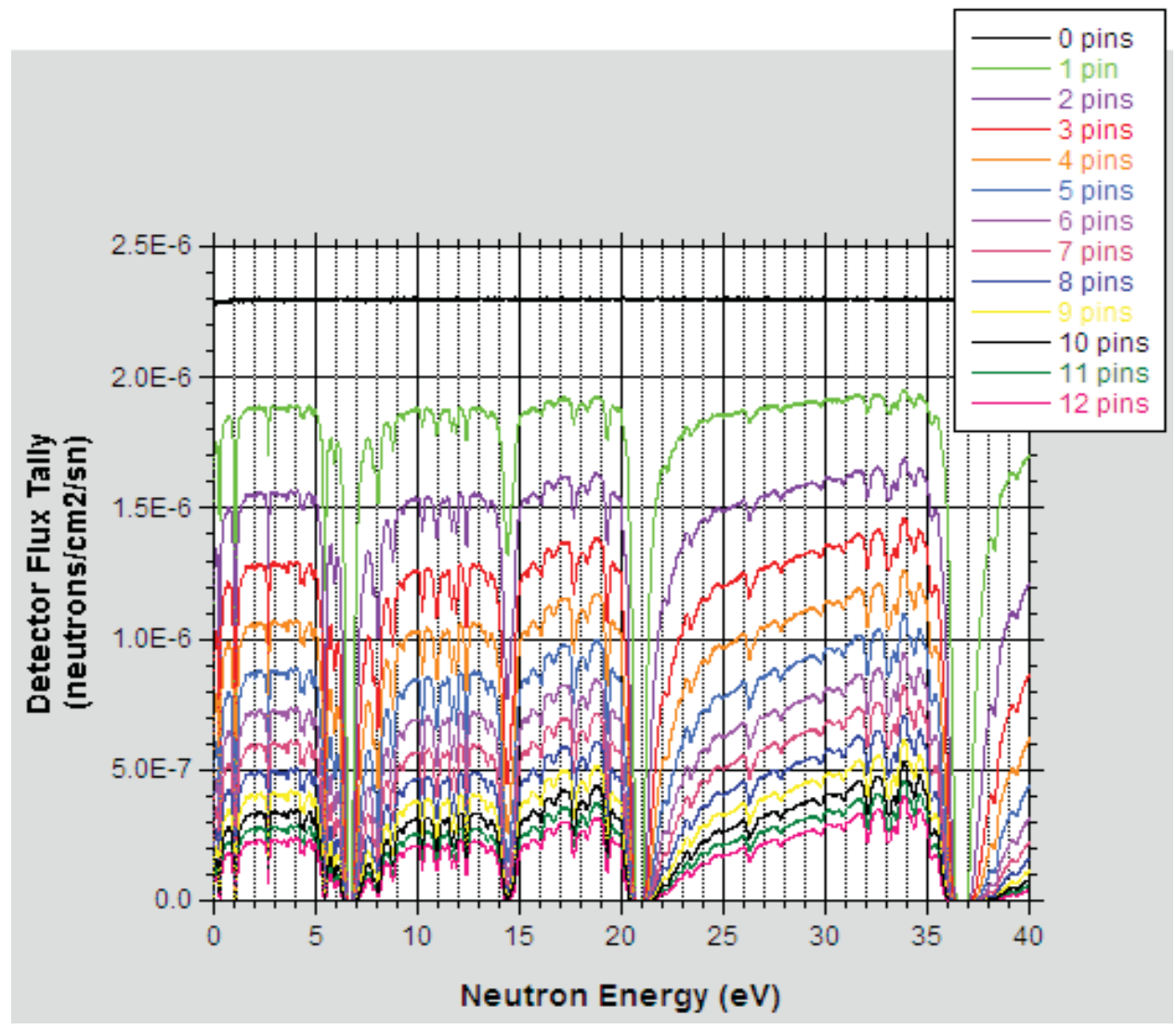

Figure 33 Neutron resonance transmission spectra through multiple spent fuel pins $(0-40 \mathrm{eV})$ at $\mathrm{y}=0.375 \mathrm{~cm}$. 


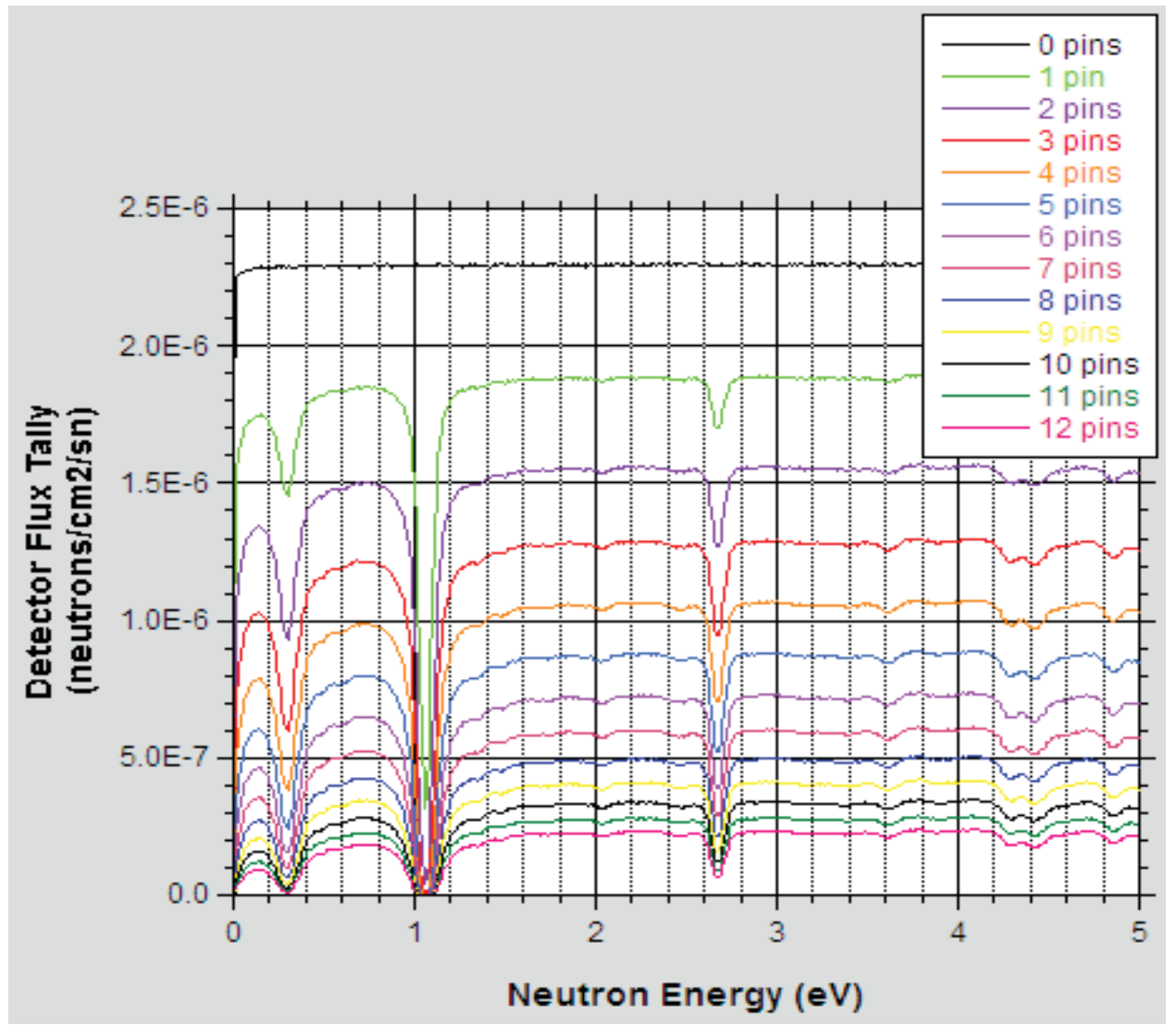

Figure 34 Neutron resonance transmission spectra through multiple spent fuel pins $(0-5 \mathrm{eV})$ at $\mathrm{y}=0.375 \mathrm{~cm}$. 


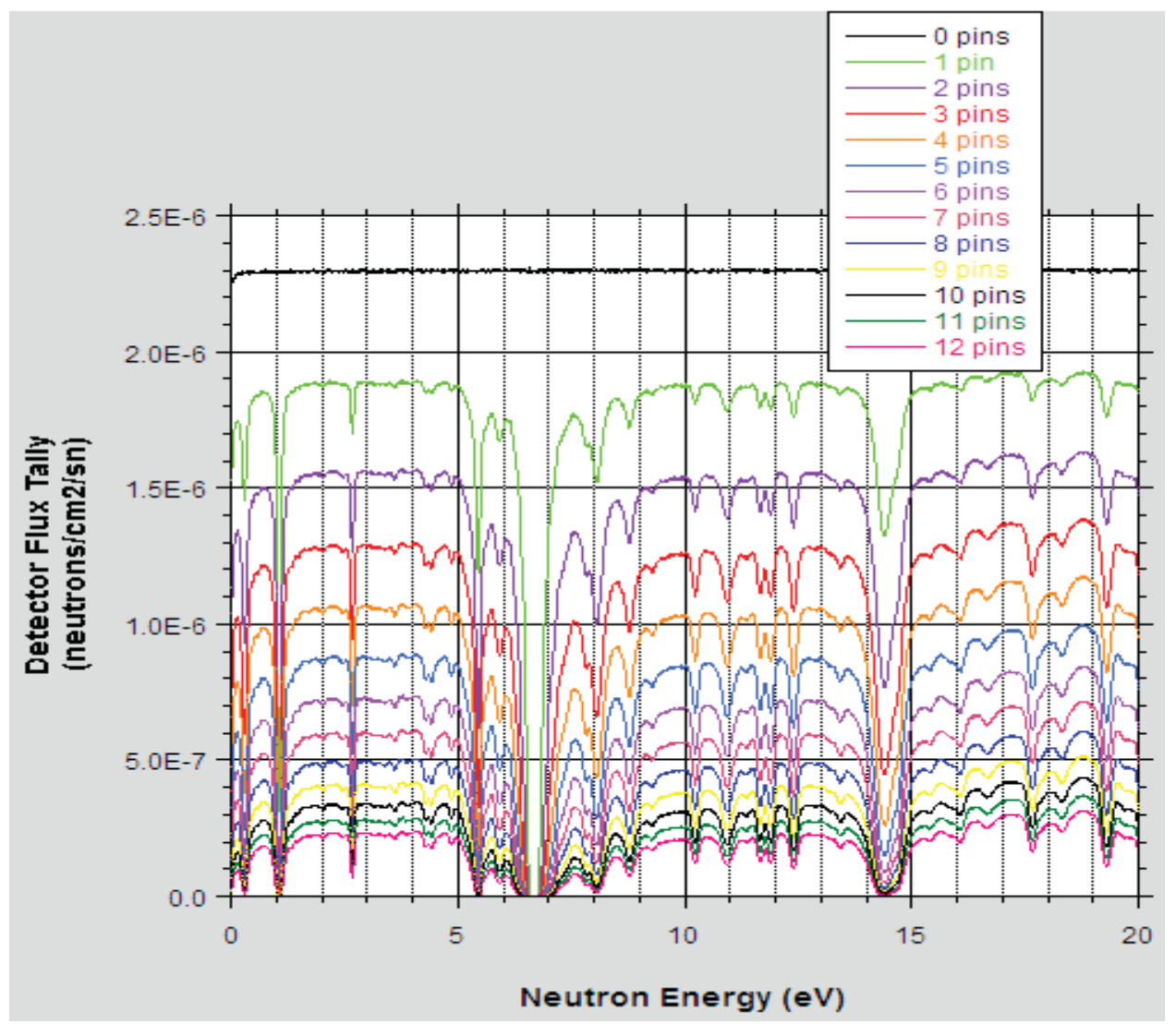

Figure 35 Neutron resonance transmission spectra through multiple spent fuel pins $(0-20 \mathrm{eV})$ at $\mathrm{y}=0.375 \mathrm{~cm}$. 


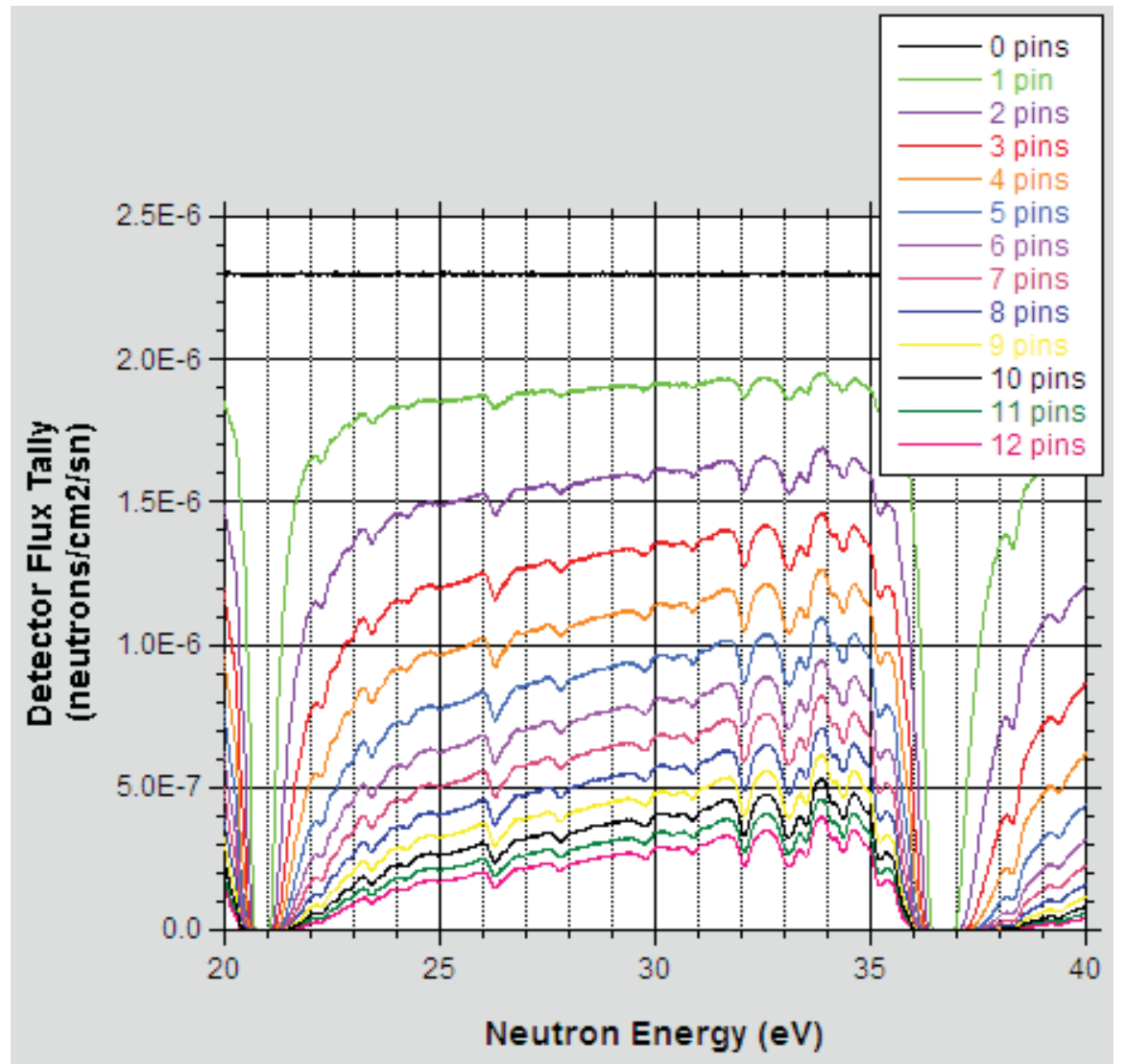

Figure 36 Neutron resonance transmission spectra through multiple spent fuel pins $(20-40 \mathrm{eV})$ at $\mathrm{y}=0.375 \mathrm{~cm}$.

In Figure 33 through Figure 36 the spacing between the transmission signals is much more uniform. This should allow better differentiation between signals and provide an opportunity to achieve better transmission penetration, possibly up to 12 pins. For a detector with high spatial resolution the on-axis $(\mathrm{y}=0.0 \mathrm{~cm})$ and off-axis $(\mathrm{y}=$ $0.100-0.395 \mathrm{~cm}$ ) transmission signals could be used together to obtain accurate 1- to 12 pin row assays.

\subsection{Vacuum Versus Air}

The baseline NRTA system design under consideration here uses an evacuated flight tube in order to minimize neutron losses and scattering (energy loss) due to elastic scatter with air molecules. Minimization of this effect is important, especially for flight tubes of considerable length. However, if the flight tube length is relatively short it may be possible to replace the vacuum with ordinary air without too great a loss in signal intensity. This section looks at calculated transmission signals for evacuated and airfilled flight tubes as a function of neutron energy and flight tube length.

Figure 37 shows MCNP-calculated neutron spectra transported down varying lengths of air-filled flight tubes, ranging from 1-10 meters, plus an evacuated flight tube as a reference case. Air density is based on standard temperature and pressure conditions. An on-axis pencil-beam neutron source without a fuel pin is assumed in the model. 


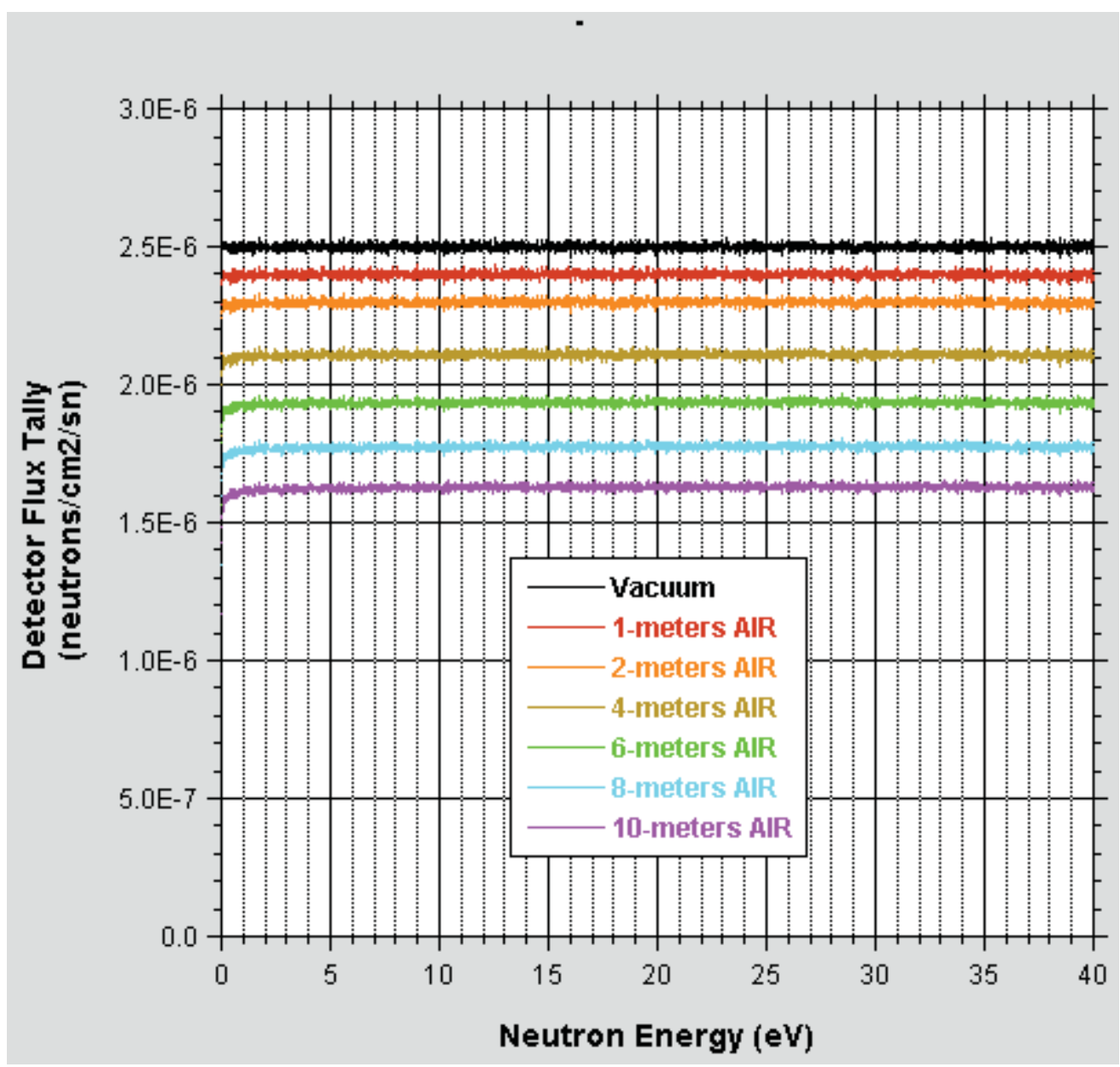

Figure 37 Neutron transmission through different flight tube lengths filled with air.

The black line shows the transmission signal for the evacuated flight tube. Maximum detector response is achieved for the evacuated system since there is no loss of neutrons. The other six transmission curves are for various air-filled flight-tube lengths. As the flight tube length is increased the transmission signal attenuation increases. For the 1-, 2-, and 4-meter lengths the neutron attenuation is 4, 8, and 16\%, respectively. Neutron attenuation in air is due mostly to elastic out-scatter by nitrogen due to its higher molecular concentration ( $80 \%$ nitrogen versus $20 \%$ oxygen) and a larger total cross section (10.6 barns for nitrogen versus 4.0 barns for oxygen). An air-filled flight tube would avoid the complexities associated with pumping and maintaining an airtight vacuum system but some loss of signal would be inevitable. If a loss of $8-16 \%$ could be tolerated, then the NRTA system flight tube would need to be relatively short, in the 2-4 meter range. The use of a relatively short flight tube should be possible for an NRTA system optimized to energies of neutron transmission in the range of $0.1-20 \mathrm{eV}$.

The results of this section indirectly address one important NRTA system design issue: the cooling of the spent fuel assembly undergoing NRTA inspection. The assembly could be placed in an air-cooled chamber during inspection, where convective air flow over the assembly could be used to control the temperature of the assembly. This could mitigate excessive pin temperatures, especially for assemblies with short 
cooling times, and possibly the need to Doppler-broaden the neutron cross sections for the assay analytical analysis.

An inspection chamber would allow sufficient space for assembly rotation and transverse movement but would be narrow enough to minimize neutron loss from air scatter. Because the air gap between pins is small $(0.31 \mathrm{~cm})$, the total transmission length of air between 17 pins would be only $5.27 \mathrm{~cm}$ ( 0.05 meters). In the case of no pins, or when the sample is removed from the inspection chamber, the thickness of the air column would be approximately $21.4 \mathrm{~cm}$ or 0.21 meters (PWR assembly dimension). Neither of these two air thicknesses would produce significant signal loss for NRTA measurements.

For the NRTA system design employing an air-filled chamber the flight tube would be divided into two sections, one in front of the chamber towards the accelerator and the second behind the chamber, attached to the neutron detectors. The aft flight-tube section could be designed as a single integrated unit containing an evacuated flight tube surrounded by neutron and gamma shielding and neutron detectors/electronics. This unit would be positioned up against a thin-walled inspection chamber and aligned with the fore flight-tube section, which would also be placed up against the inspection chamber wall.

\subsection{Rhodium-103}

At the start of this project the presence of the fission product ${ }^{103} \mathrm{Rh}$ in spent fuel was identified as a potential concern. Rhodium-103 has a radiative capture resonance at $1.25 \mathrm{eV}$ that is in the immediate vicinity of the ${ }^{240} \mathrm{Pu}$ resonance at $1.07 \mathrm{eV}$. This is the only resonance for ${ }^{240} \mathrm{Pu}$ in the $0-40 \mathrm{eV}$ range. Interference between these two resonances could degrade the NRTA ${ }^{240} \mathrm{Pu}$ assay signal. Adding to the concern, ${ }^{103} \mathrm{Rh}$ was not included in the LANL spent-fuel material libraries. Further, ${ }^{103} \mathrm{Rh}$ is known to be a strong thermal neutron absorber in irradiated fuel.

In order to evaluate the effect of ${ }^{103} \mathrm{Rh}$ on the NRTA transmission spectra and to determine whether the ${ }^{240} \mathrm{Pu}$ resonance $(1.07 \mathrm{eV})$ would be substantially degraded by the presence of the ${ }^{103} \mathrm{Rh}$ in the spent fuel, additional spent-fuel depletion calculations were needed to establish the ${ }^{103} \mathrm{Rh}$ concentration as a function of burnup. The ${ }^{103} \mathrm{Rh}$ concentrations could then simply be added to specific LANL spent fuel models as needed. Rather than reproduce the entire LANL 64 spent fuel parametric compositions, a reduced set of spent fuel compositions was developed by INL which included burnups of 15, 30, 45, and $60 \mathrm{GWD} / \mathrm{MTU}$ at a single enrichment and cooling time. Assumptions that went into the INL depletion calculations included: (1) single PWR fuel pin, (2) 3.0 wt $\%$ enrichment, (3) 15, 30, 45, and 60 GWD/MTU burnups, (4) 1-year cooling time, and (5) uniform and average pin power of $66.95 \mathrm{~kW} / \mathrm{rod}$. Note that the 1-year cooling time allows all the ${ }^{103} \mathrm{Ru}$ (39.27 day half-life) to decay to ${ }^{103} \mathrm{Rh}$. The ORIGEN2.2 depletion code was used to perform the INL burnup calculations using a standard PWR cross section library.[24]

Comparison of the LANL and INL spent-fuel compositions showed the major actinide and fission product atom fractions were in excellent agreement. The ${ }^{103} \mathrm{Rh}$ spent fuel concentrations were then extracted from the INL calculations and simply inserted into the appropriate LANL spent-fuel compositions as an atom fraction consistent with the LANL material composition format. Transmission spectra at the four different 
burnups were then calculated and comparisons made between spectra with and without the ${ }^{103} \mathrm{Rh}$ isotope.

Figure 38 and Figure 39 are neutron transmission spectra for the 15 GWD/MTU burnup case with and without ${ }^{103} \mathrm{Rh}$. Figure 38 shows the spectrum over the $0-40 \mathrm{eV}$ energy range. No differences are discernable between the " $103 \mathrm{Rh}$ " and "no ${ }^{103} \mathrm{Rh}$ " cases over this broad energy range. The next figure zooms on ${ }^{240} \mathrm{Pu}$ and ${ }^{103} \mathrm{Rh}$ resonances in the vicinity of $1.0 \mathrm{eV}$.

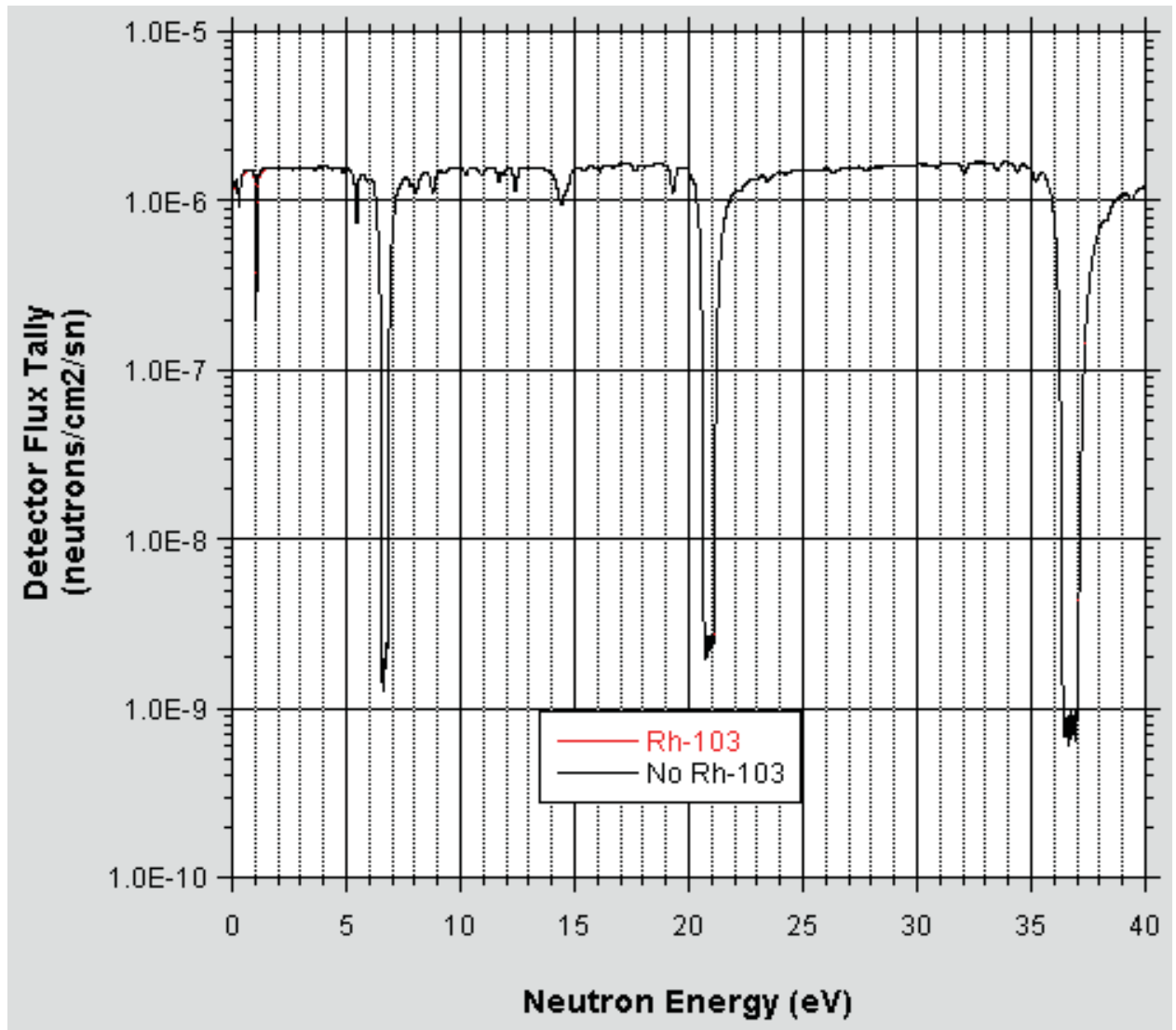

Figure 38 Transmission spectra for $15 \mathrm{GWD} / \mathrm{MTU}$ burnup with and without ${ }^{103} \mathrm{Rh}$ over the 0-40 eV energy range. 
Figure 39 is the same as Figure 38 except it zooms in on the $0-2 \mathrm{eV}$ energy range in order to see the difference between the " ${ }^{~}{ }^{103} \mathrm{Rh}$ " and "no ${ }^{103} \mathrm{Rh}$ " cases. Both figures are plotted on semi-log scales in order to improve differentiation of the two signals. No ${ }^{103} \mathrm{Rh}$ interference is observed with the ${ }^{240} \mathrm{Pu}$ resonance.

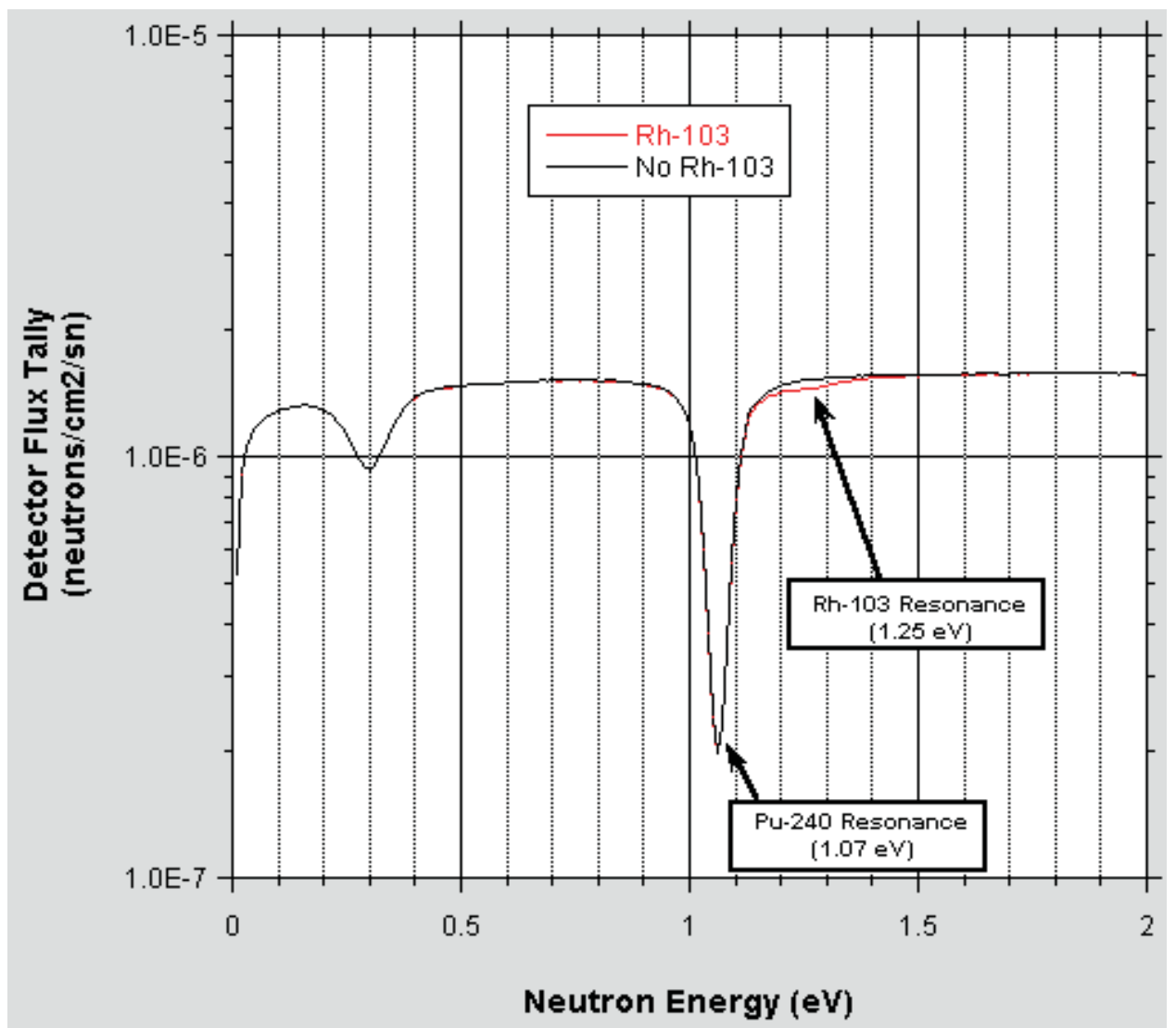

Figure 39 Transmission spectra for $15 \mathrm{GWD} / \mathrm{MTU}$ burnup with and without ${ }^{103} \mathrm{Rh}$ over the 0-2 eV energy range.

Figure 40 and Figure 41 are similar to Figure 39 but assume 30 and 45 GWD/MTU burnups, respectively. The ${ }^{103} \mathrm{Rh}$ resonance interference effect is still small and inconsequential. 


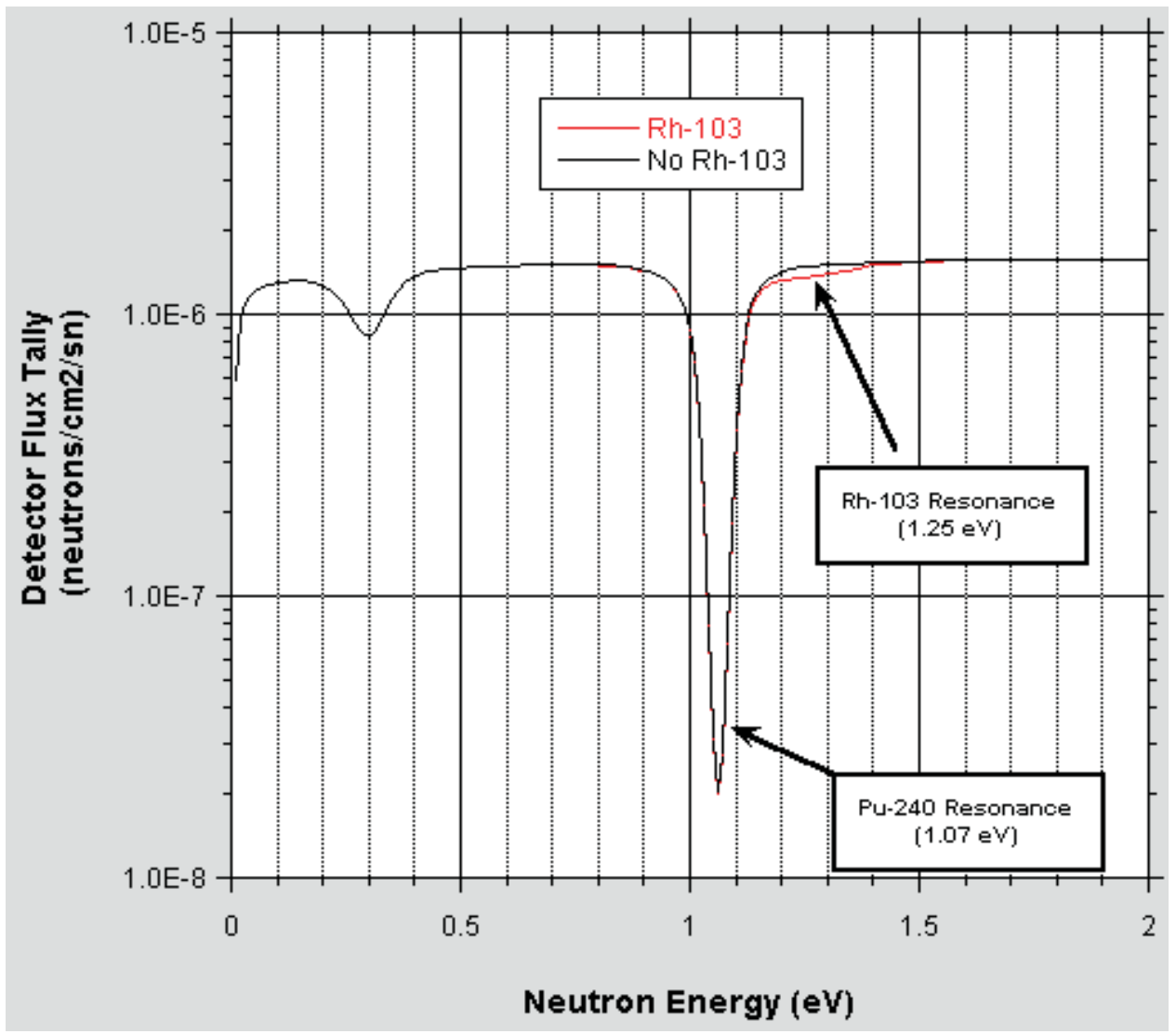

Figure 40 Transmission spectra for $30 \mathrm{GWD} / \mathrm{MTU}$ burnup with and without ${ }^{103} \mathrm{Rh}$ over the 0-2 eV energy range. 


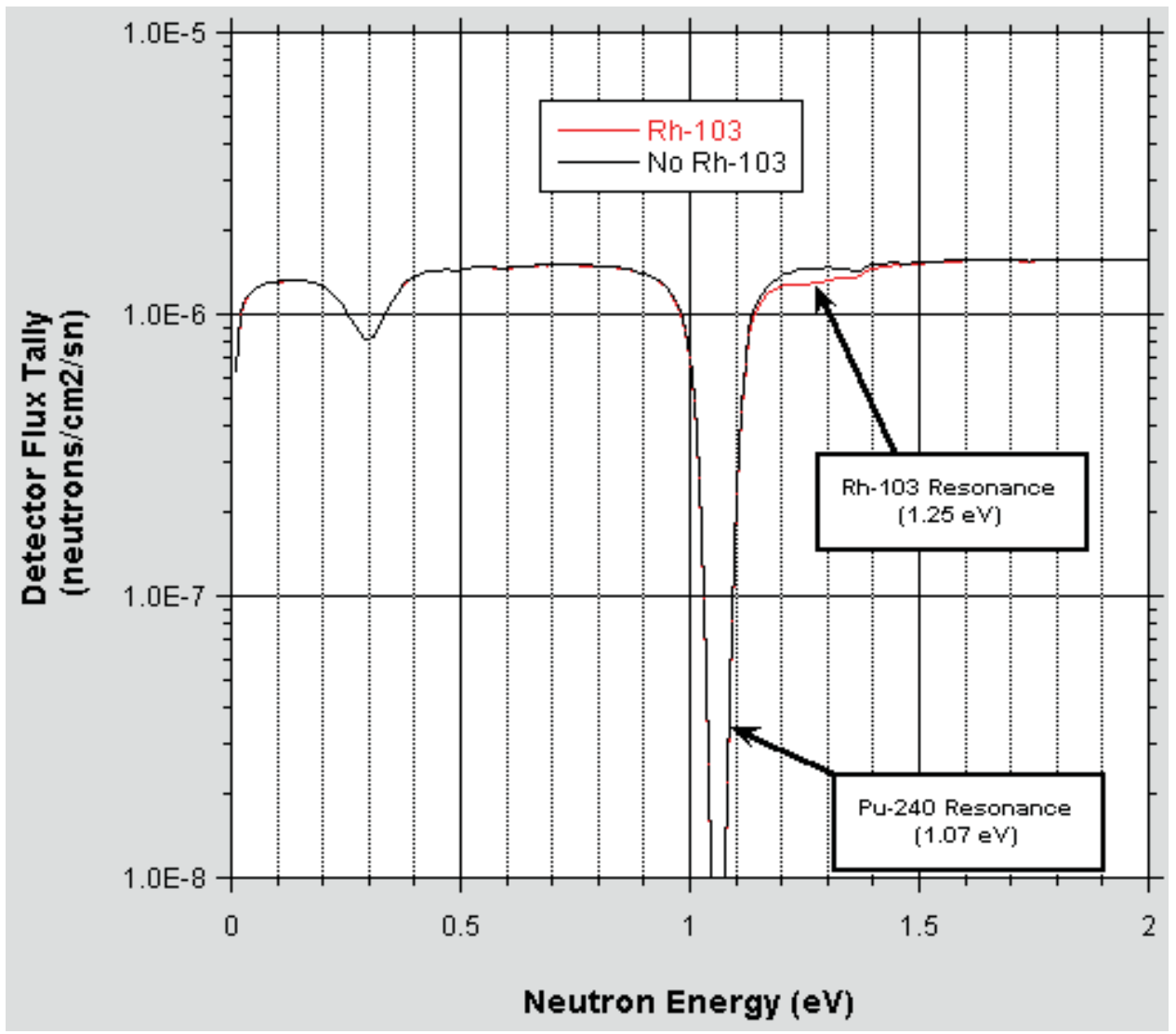

Figure 41 Transmission spectra for $45 \mathrm{GWD} / \mathrm{MTU}$ burnup with and without ${ }^{103} \mathrm{Rh}$ over the 0-2 eV energy range.

The $60 \mathrm{GWD} / \mathrm{MTU}$ burnup transmission spectra has been omitted. The resonance interference effect at $60 \mathrm{GWD} / \mathrm{MTU}$ burnup is also small and inconsequential, as can be extrapolated from Figure 39 through Figure 41.

From these calculated transmission spectra the ${ }^{103} \mathrm{Rh}$ resonance does not appear to significantly interfere with the ${ }^{240} \mathrm{Pu}$ resonance over the $0-60 \mathrm{GWD} / \mathrm{MTU}$ burnup range. Several reasons can explain this conclusion: (1) the ${ }^{240} \mathrm{Pu}$ resonance energy is at $1.07 \mathrm{eV}$ and the ${ }^{103} \mathrm{Rh}$ resonance is at $1.25 \mathrm{eV}$, so the two resonances do not actually lie directly on top of each other, and the widths of both are relatively narrow, (2) the ${ }^{240} \mathrm{Pu}$ resonance has a peak total cross section of approximately 100,000 barns whereas ${ }^{103} \mathrm{Rh}$ is only 5,000 barns, so the ${ }^{240} \mathrm{Pu}$ resonance can absorb more neutrons and create a much deeper resonance depression, and (3) the spent fuel concentrations of both ${ }^{240} \mathrm{Pu}$ and ${ }^{103} \mathrm{Rh}$ are comparable over the $0-60 \mathrm{GWD} / \mathrm{MTU}$ range with the ${ }^{240} \mathrm{Pu}$ concentration being slightly higher. Hence, it is concluded that although ${ }^{103} \mathrm{Rh}$ will be discernable by the NRTA technique, and even possibly useful as a high-burnup indicator, it is not expected to pose a resonance interference problem with ${ }^{240} \mathrm{Pu}$. The ${ }^{240} \mathrm{Pu}$ resonance at $1.07 \mathrm{eV}$ should be highly resolvable with the NRTA technique and be very useful as a spent fuel plutonium assay measurement and burnup estimator. 


\subsection{Spent-Fuel Neutron Emission}

Neutrons are emitted naturally from spent fuel assemblies, primarily from $(\alpha, n)$ reactions and spontaneous fission (SF). These background neutrons are a steady and continuous source of fast neutrons that can create background counts in a nearby detector used for NRTA measurements. In addition, these neutrons can induce fission in the assembly and create additional fission neutrons through assembly multiplication. These background neutrons could pose a concern for the NRTA interrogating neutron transmission signal. This section evaluates the potential impact of these background neutrons on the NRTA transmission signal and helps define shielding requirements for an NRTA conceptual system design.

Helium nuclei, or alpha particles, are given off naturally from the decay of virtually all of the spent fuel actinides with the exception of ${ }^{241} \mathrm{Pu},{ }^{236} \mathrm{~Np}$, and ${ }^{242} \mathrm{Am}$, which decay through other mechanisms. These alpha particles interact with the oxygen in the $\mathrm{UO}_{2}$ and produce approximately 0.07 neutrons per million alphas.[25]

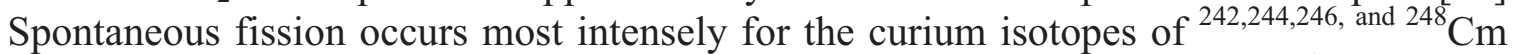

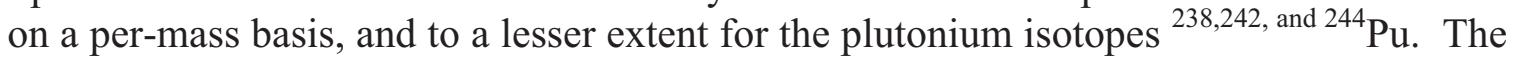
first step in evaluating the potential effect of these background neutrons is to estimate the source strengths of the $(\alpha, n)$ and SF emissions. Table 1 gives calculated estimates of the $(\alpha, n)$ and SF neutron components along with their sum, or total number of neutrons emitted from an assembly. Basic nuclear and derived data were taken from three sources.[25-27]. The data in Table 1 have also been compared with reference 28 and the two sources are in very good agreement. The values in Table 1 are plotted in Figure 42 through Figure 46 for different cooling times and burnups.

Table 1 Spent fuel assembly neutron source strength or emission rate (neutrons/second).

\begin{tabular}{|c|c|c|c|c|c|}
\hline $\begin{array}{c}\text { Burnup } \\
\text { (GWD/MTU) }\end{array}$ & $\begin{array}{c}{ }^{235} \mathrm{U} \\
\text { Enrichment } \\
(w t \%)\end{array}$ & $\begin{array}{c}\text { Cooling } \\
\text { Time } \\
\text { (yr) }\end{array}$ & $\begin{array}{c}(\alpha, n) \\
(n / s e c)\end{array}$ & $\begin{array}{c}\text { SF } \\
(\mathbf{n} / \text { sec) }\end{array}$ & $\begin{array}{c}\text { Total } \\
(\mathrm{n} / \mathrm{sec})\end{array}$ \\
\hline 15 & 3.0 & 1 & $2.5293 \mathrm{E}+06$ & $5.7803 \mathrm{E}+06$ & $8.3096 \mathrm{E}+06$ \\
\hline 15 & 3.0 & 5 & $1.6369 \mathrm{E}+06$ & $5.0342 \mathrm{E}+06$ & $6.6711 \mathrm{E}+06$ \\
\hline 15 & 3.0 & 20 & $2.4198 \mathrm{E}+06$ & $3.0630 \mathrm{E}+06$ & $5.4827 \mathrm{E}+06$ \\
\hline 15 & 3.0 & 80 & $2.8485 \mathrm{E}+06$ & $7.7115 \mathrm{E}+05$ & $3.6197 \mathrm{E}+06$ \\
\hline 30 & 3.0 & 1 & $1.7205 \mathrm{E}+07$ & $1.4932 \mathrm{E}+08$ & $1.6652 \mathrm{E}+08$ \\
\hline 30 & 3.0 & 5 & $7.1961 \mathrm{E}+06$ & $1.2838 \mathrm{E}+08$ & $1.3558 \mathrm{E}+08$ \\
\hline 30 & 3.0 & 20 & $7.9532 \mathrm{E}+06$ & $7.3115 \mathrm{E}+07$ & $8.1068 \mathrm{E}+07$ \\
\hline 30 & 3.0 & 80 & $7.3881 \mathrm{E}+06$ & $9.0026 \mathrm{E}+06$ & $1.6391 \mathrm{E}+07$ \\
\hline 45 & 3.0 & 1 & $4.6577 \mathrm{E}+07$ & $7.8497 \mathrm{E}+08$ & $8.3154 \mathrm{E}+08$ \\
\hline 45 & 3.0 & 5 & $2.1841 \mathrm{E}+07$ & $6.7486 \mathrm{E}+08$ & $6.9670 \mathrm{E}+08$ \\
\hline 45 & 3.0 & 20 & $1.8385 \mathrm{E}+07$ & $3.8406 \mathrm{E}+08$ & $4.0245 \mathrm{E}+08$ \\
\hline 45 & 3.0 & 80 & $1.1904 \mathrm{E}+07$ & $4.6738 \mathrm{E}+07$ & $5.8641 \mathrm{E}+07$ \\
\hline 60 & 3.0 & 1 & $8.6970 \mathrm{E}+07$ & $2.1543 \mathrm{E}+09$ & $2.2413 \mathrm{E}+09$ \\
\hline 60 & 3.0 & 5 & $4.8208 \mathrm{E}+07$ & $1.8541 \mathrm{E}+09$ & $1.9024 \mathrm{E}+09$ \\
\hline 60 & 3.0 & 20 & $3.5053 \mathrm{E}+07$ & $1.0612 \mathrm{E}+09$ & $1.0963 \mathrm{E}+09$ \\
\hline 60 & 3.0 & 80 & $1.6376 \mathrm{E}+07$ & $1.4126 \mathrm{E}+08$ & $1.5764 \mathrm{E}+08$ \\
\hline
\end{tabular}




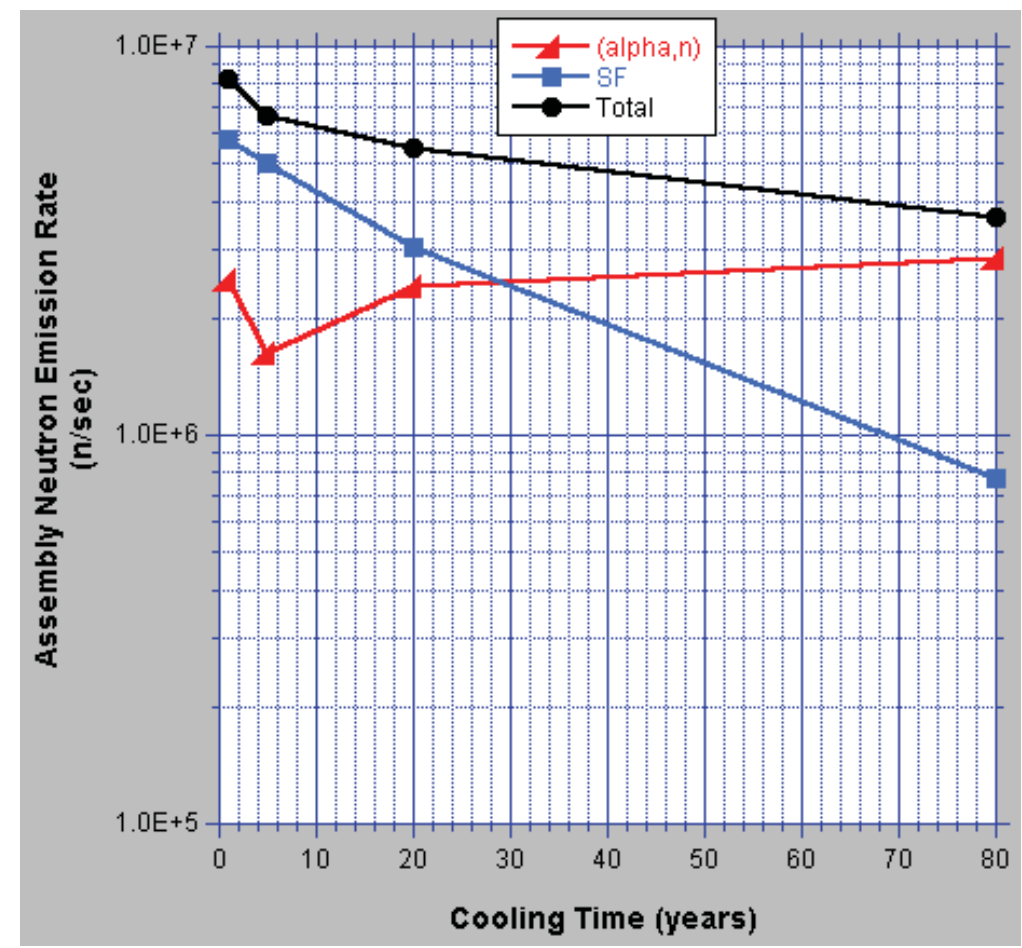

Figure 42 Total neutron emission rate versus cooling time for a single PWR spent fuel assembly (15 GWD/MTU).

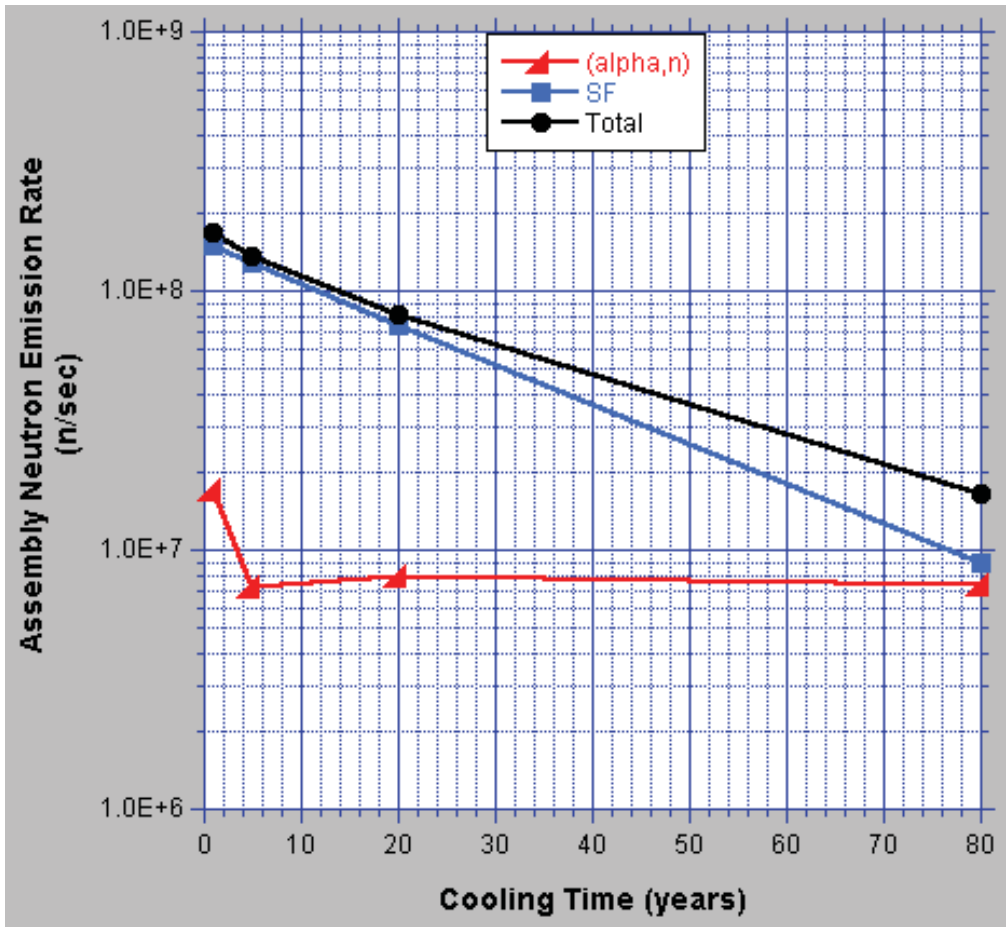

Figure 43 Total neutron emission rate versus cooling time for a single PWR spent fuel assembly (30 GWD/MTU). 


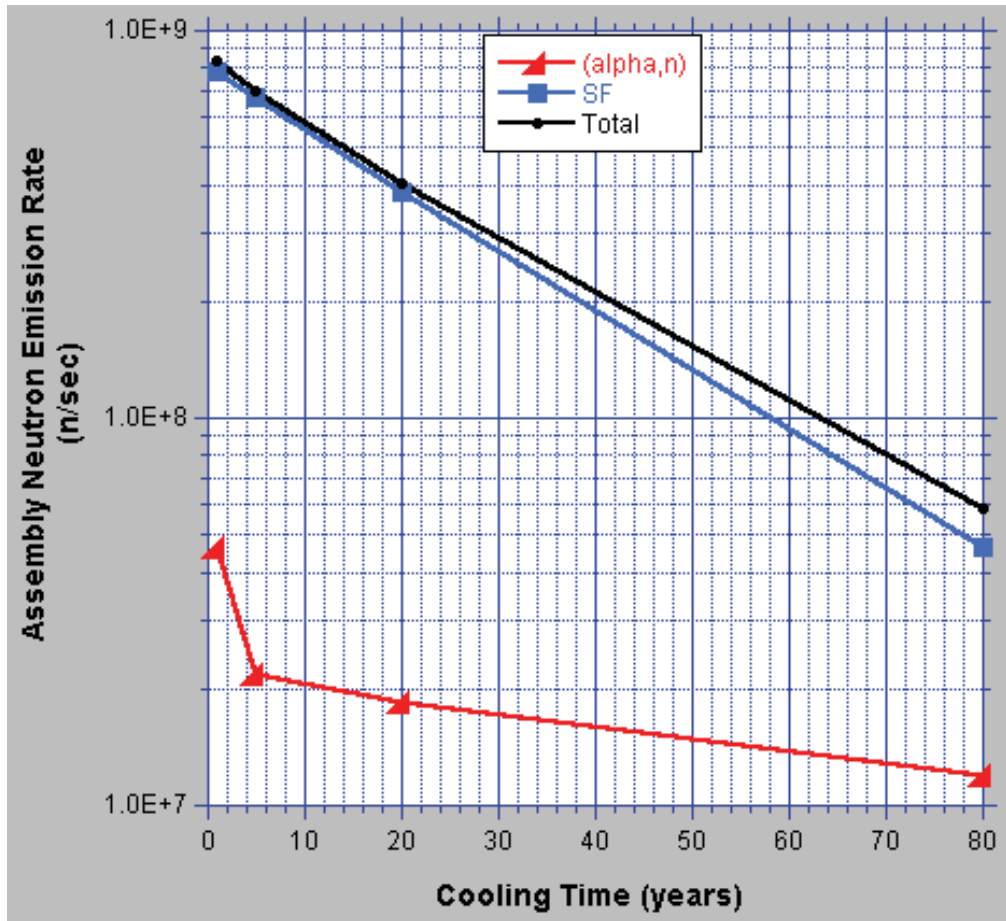

Figure 44 Total neutron emission rate versus cooling time for a single PWR spent fuel assembly (45 GWD/MTU).

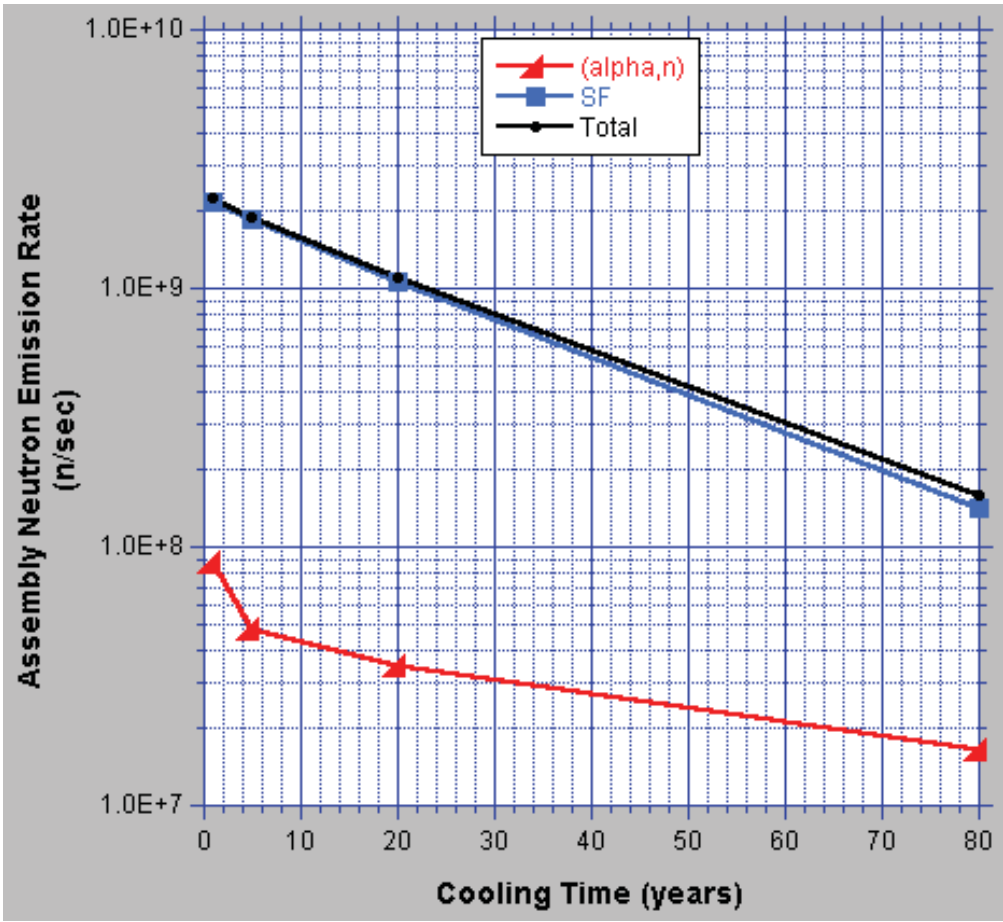

Figure 45 Total neutron emission rate versus cooling time for a single PWR spent fuel assembly (60 GWD/MTU). 


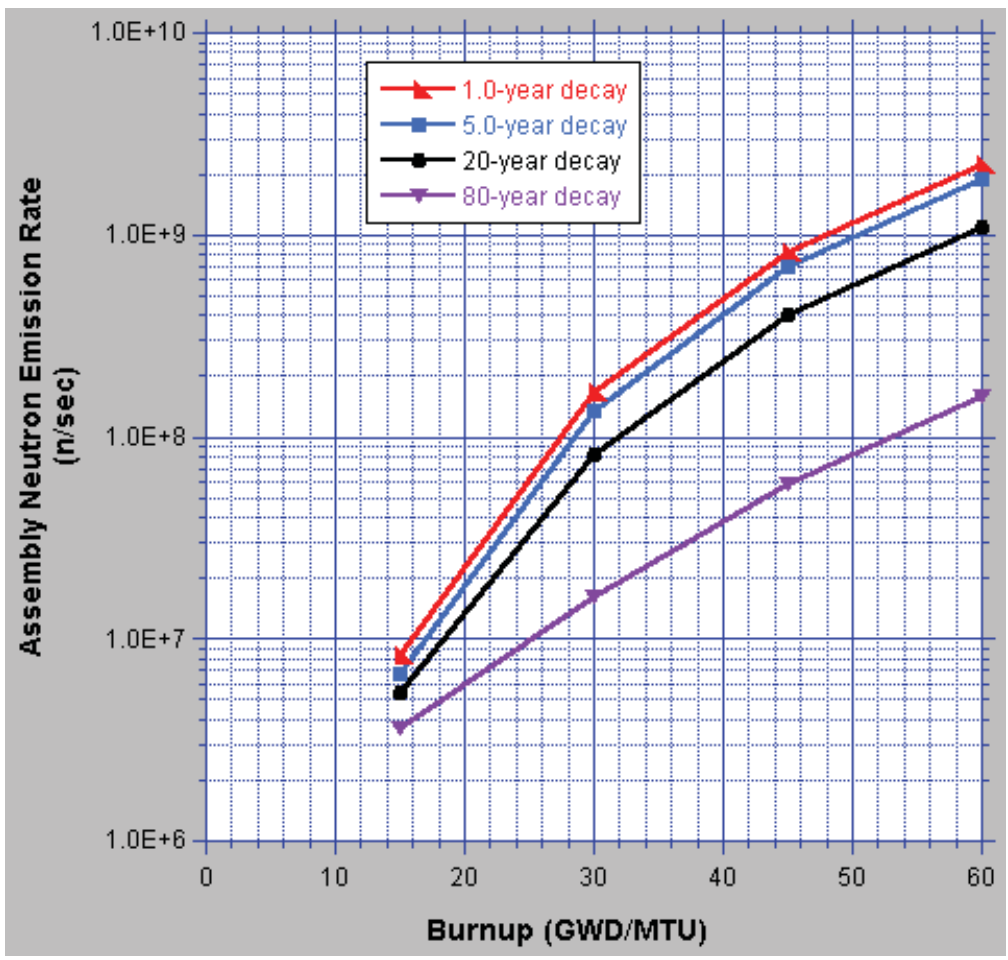

Figure 46 Total neutron emission rate for a single PWR spent fuel assembly as a function of burnup.

The total neutron emission rate from a spent fuel assembly increases with burnup and decreases with cooling time. The SF component is the dominant contributor to the neutron emission rate for all burnups and cooling times except at the low $15 \mathrm{GWD} / \mathrm{MTU}$ burnup and long 30-80 years cooling times, where the $(\alpha, n)$ component is larger.

The total neutron-emission rate ranges from $3.62 \times 10^{6}$ to $2.24 \times 10^{9} \mathrm{n} / \mathrm{sec}$ for the $3.0 \mathrm{wt} \%{ }^{235} \mathrm{U}$ initial enrichment assembly (see Figure 46). Emission rates, however, are also a function of the initial uranium assembly enrichment; the neutron emission rates increase with decreasing enrichment.[29] Going from 3\% to $2 \%$ enrichment would increase the emission rate for a maximum burnup (60 GWD/MTU) spent fuel assembly with 1 -year cooling time by a factor of 1.55 . Decreasing the cooling time from 1 year to 28 days or 7 days would again further increase the total neutron emission rate by factors of 1.16 and 1.18, respectively. Therefore, an expected maximum neutron-emission rate for a maximized spent fuel assembly (60 GWD/MTU, 7-day cooling, 2\% enrichment) would be expected to be approximately $1.55 \times 1.18 \times\left(2.24 \times 10^{9}\right)=4.1 \times 10^{9} \mathrm{n} / \mathrm{sec}$.

The $(\alpha, n)$ and SF neutrons can also induce fission in the spent fuel assembly and create additional multiplication neutrons. Spent fuel assembly multiplication factors have been calculated for assemblies in vacuum; the NRTA technique will place spent fuel assemblies in either an evacuated or air-filled chamber. Either way, vacuum or air-filled will have negligible effect on the assembly multiplication factors.

Table 2 gives the MCNPX-calculated k-effective and multiplication factors for a single assembly with varying enrichments and burnups: fresh $\mathrm{UO}_{2}$ fuel with enrichments of $2,3,4,5$, and $6 \mathrm{wt} \%$ and spent fuel with $3.0 \mathrm{wt} \%$ initial enrichment, burnups of 15,30 , 45 , and $60 \mathrm{GWD} / \mathrm{MTU}$, and a 1 -year cooling time. 
Table 2 Calculated k-effectives and multiplication factors for a single assembly in vacuum.

\begin{tabular}{ccccc}
\hline $\begin{array}{c}\text { MCNPX } \\
\text { Case }\end{array}$ & $\begin{array}{c}{ }^{235} \mathbf{U} \\
\text { Enrichment } \\
(\mathbf{w t} \%)\end{array}$ & $\begin{array}{c}\text { Burnup } \\
\text { (GWD/MTU) }\end{array}$ & K-effective & $\begin{array}{c}\text { Neutron } \\
\text { Multiplication } \\
\text { Factor }\end{array}$ \\
\hline kns2 & 2.0 & 0.0 & 0.08025 & 1.08725 \\
kns0 & 3.0 & 0.0 & 0.08426 & 1.09201 \\
kns3 & 4.0 & 0.0 & 0.08738 & 1.09575 \\
kns4 & 5.0 & 0.0 & 0.09115 & 1.10029 \\
kns1 & 6.0 & 0.0 & 0.09508 & 1.10507 \\
kns15 & 3.0 & 15.0 & 0.08197 & 1.08929 \\
kns30 & 3.0 & 30.0 & 0.07999 & 1.08694 \\
kns45 & 3.0 & 45.0 & 0.07850 & 1.08519 \\
kns60 & 3.0 & 60.0 & 0.07747 & 1.08397 \\
\hline
\end{tabular}

The neutron multiplication factors in Table 2 are relatively small. The fresh-fuel multiplication factors range from 1.08725 to 1.10507 with the highest enrichment $(6 \%)$ producing the largest multiplication factor, as expected. The spent-fuel multiplication factors are smaller than the fresh $3 \%$ fuel and decrease slightly with burnup. For the NRTA assessment the maximum neutron assembly emission rate $\left(4.1 \times 10^{9} \mathrm{n} / \mathrm{sec}\right)$ derived above can now be conservatively multiplied by a factor of 1.11 in order to account for assembly neutron multiplication. Therefore, a maximum neutron emission rate of $4.55 \times$ $10^{9} \mathrm{n} / \mathrm{sec}$ from a single PWR assembly will be used in the following NRTA assessment of the impact of background neutrons on the neutron detectors. In addition, the neutrons will be assumed to be emitted continuously and uniformly in all directions (isotropic emission) and uniformly over the assembly fuel volume.

From a solid-angle view point the flight-tube distance between the assembly and the neutron detectors will be assumed to be 2,4 , or 10-meters in length; a 10-meter long flight tube is probably the longest flight-tube length that would be needed to resolve NRTA TOF neutrons with energies between $0-20 \mathrm{eV}$. It is further assumed that the detector area is $9 \mathrm{~cm}^{2}$. The probabilities of a neutron striking the detector are $1.8 \times 10^{-5}$, $4.5 \times 10^{-6}$, and $7.2 \times 10^{-7}\left(9 \mathrm{~cm}^{2} / 4 \pi / 1000^{2} \mathrm{~cm}^{2}\right)$ for 2-, 4-, and 10-meter stand-off distances, respectively. Therefore, approximately $8.19 \times 10^{4}, 2.05 \times 10^{4}$, and $3.26 \times 10^{3}$ $\mathrm{n} / \mathrm{sec}\left(7.2 \times 10^{-7} \times 4.55 \times 10^{9}\right)$ will strike the detector, and for micro-second time bin widths this amounts to $0.082,0.021$, and 0.0033 neutrons per bin, or 1 neutron per bin per 12,48 , and 303 accelerator cycles, respectively.

The above analysis assumes the entire spent-fuel-assembly source to be a point source. In reality, the background neutron source is distributed over the entire assembly fueled volume and approximately uniformly over both the 3.66 meter length and the 21.4 $\times 21.4 \mathrm{~cm}$ cross section of the assembly. The neutron background source can therefore be further reduced if neutron shielding is applied around the flight tube and between the assembly and the detectors. Then neutrons emitted off-axis of the flight tube will have great difficulty scattering into the detector solid angle. Based on MCNP model transport calculations only spent-fuel neutrons emitted in the spent fuel, on the flight-tube axis, and in the solid angle direction of the detectors will actually reach the detectors. The 
probability that neutrons emitted in other regions of the assembly scatter into the detectors is negligible. Neutrons from pins lined up with the flight tube/detector (maximum 17 ), and then only a small axial portion of those fuel rods ( $\sim 10 \mathrm{~cm}$ in height), will contribute to the detector counts. Based on these refinements the backgroundneutron source strength can be significantly reduced: a still conservative estimate of the emission rate is $(17 \mathrm{pins} / 264 \mathrm{pins}) \times(10 \mathrm{~cm} / 366 \mathrm{~cm}) \times\left(4.55 \times 10^{9} \mathrm{n} / \mathrm{s}\right)=8.0 \times 10^{6} \mathrm{n} / \mathrm{s}$.

Considering the detector's solid angle, isotropic emission, and 2-, 4-, or 10-meter stand-off lengths with a $9-\mathrm{cm}^{2}$ detector area, the number of neutrons striking the detector are only 144,36 , and $5.8 \mathrm{n} / \mathrm{sec}\left(7.2 \times 10^{-7} \times 8.0 \times 10^{6}\right)$, respectively for these distances. For micro-second time bins, this amounts to $1.44 \times 10^{-4}, 3.6 \times 10^{-5}$, and $5.73 \times 10^{-6}$ neutrons per bin, respectively.

The conclusion to be drawn here is that with efficient neutron shielding (for example, borated-polyethylene) between the fuel assembly and the detectors, the background neutron source from the spent fuel assembly should not have a significant impact on the NRTA neutron transmission signal. Neutron shielding around the detectors will also be required in order minimize room-scattered and back-scattered neutrons.

\subsection{Spent-Fuel Gamma Emission}

No work has been performed to date to evaluate the expected intense gamma field emission from spent PWR fuel assemblies and their impact on NRTA measurements, in particular the high-burnup assemblies (45-60 GWD/MTU) with short cooling times. Gamma shielding will be needed around the aft flight tube, shielding that will need to be integrated with the neutron shielding, in order to minimize gamma radiation impacting the neutron detectors. Detector design, gamma-shield design, and minimization of the solid angle viewed by the detectors of the spent fuel assembly will eventually need to be addressed in the conceptual NRTA system design.

\subsection{Fuel-Pin Diversion}

The three NGSI diversion scenarios involving 8, 24, and 40 pins in the PWR assembly have not been evaluated yet. However, based on the preliminary conceptual studies performed so far, removing this many pins from the assembly and replacing them with depleted-uranium oxide surrogate pins should be readily detectable by the NRTA technique. Surrogate materials other than depleted-uranium oxide would also be readily detectable by NRTA. For example, other possible heavy metal surrogates might include lead, bismuth, and tungsten. Metal rods containing these metal isotopes will have either very definitive resonances, or possibly, no resonance structure at all in the $0-20 \mathrm{eV}$ energy range. Either way, differences in transmission spectra will show up as new or abnormal resonances and reduced plutonium, ${ }^{235} \mathrm{U}$, and fission-product depressions.

Inclusion of the previously mentioned fission products with resonance structure in the $0-20 \mathrm{eV}$ range into a replacement fuel pin during a diversion event would be very difficult to carry out. The correct isotopic ratios and absolute concentrations for each of ${ }^{99} \mathrm{Tc},{ }^{103} \mathrm{Rh},{ }^{131} \mathrm{Xe},{ }^{133} \mathrm{Cs},{ }^{145} \mathrm{Nd}$, and ${ }^{152} \mathrm{Sm}$ would need to be added to the replacement fuel. One of the resonance fission products that would be particularly difficult to use is ${ }^{131} \mathrm{Xe}$, a noble gas that would volatilize during reprocessing and would be difficult to put back into a surrogate fuel. Xenon-131 is readily detectable by the NRTA technique and any deviation in its isotopic concentration could be an easy diversion flag. A single fuel pin 
missing this isotope would result in an $8.3 \%$ loss in total concentration for a row of 12 pins and greater percentages for rows with fewer pins. A similar percent loss would be expected for extracted plutonium isotopes as well.

\subsection{Missing Fuel Pins}

Missing fuel pins may be readily detected through assembly weight measurements. However, if it is necessary to locate the missing pins the NRTA technique may be suited for this determination using two possible methods. The first method simply involves comparing "normal mode" transmission spectra for known numbers of fuel pins in a row and identifying signals that are inconsistent (resonance depressions are too shallow). This is a quick and easy method.

A second method would use higher-energy neutrons with energies between 1-20 $\mathrm{MeV}$. Neutrons at these energies have lower total interaction cross-sections and are therefore more penetrating than the thermal neutrons needed for NRTA. Fast neutrons used in this way would produce a fast-neutron radiograph, or a tomograph if multiple views are taken. This method lends itself to the NRTA technique quite readily by simply removing the low-Z neutron-moderating filter. Removal of the neutron moderating filter allows fast photoneutrons to be emitted from the bremsstrahlung-to-neutron converter. These fast neutrons are considerably higher in energy than the thermalized neutrons $(0-$ $20 \mathrm{eV}$ ) used in the NRTA "normal mode." The NRTA system operating without the low$\mathrm{Z}$ moderator (slowing-down) filters will be referred to here as the "fast mode" of operation.

In the "fast mode," for example, if a deuterium or beryllium bremsstrahlung-toneutron converter were used with maximum accelerator electron energy of $10 \mathrm{MeV}$, the emitted photoneutrons would have energies ranging from $\leq 3.6 \mathrm{MeV}$ and $\leq 7.5 \mathrm{MeV}$, respectively. For a $15 \mathrm{MeV}$ maximum accelerator electron beam energy the energy of the photoneutrons for these two converters could be further raised to $\leq 6.4 \mathrm{MeV}$ and $\leq 12.5$ $\mathrm{MeV}$, respectively. The ability to readily adjust the accelerator's maximum electron energy should be possible and can be included as an NRTA system design goal.[2]

The magnitude of the total cross section for fast neutrons in the $\mathrm{MeV}$ energy range is typically lower than that for thermal and epithermal neutrons. This is true for oxygen and uranium, oxygen and uranium being the main constituents in $\mathrm{UO}_{2}$ fuel. The total cross section for oxygen is 4.0 barns over the entire $1 \mathrm{eV}-0.2 \mathrm{MeV}$ energy range and, for uranium, approximately 10.0 barns. For energies above $1 \mathrm{MeV}$ both cross sections tend to decrease. Table 3 gives the $\mathrm{UO}_{2}$ macroscopic total cross section, neutron mean-free-path (mfp), and the equivalent number of PWR fuel pins per mfp as a function of neutron energy for 10 and $20 \mathrm{MeV}$, plus the $1.0 \mathrm{eV}-0.2 \mathrm{MeV}$ energy range.

Table 3 Total cross section and mean-free-path ( $\mathrm{mfp}$ ) for $\mathrm{UO}_{2}$ fuel.

\begin{tabular}{cccc}
\hline $\begin{array}{c}\text { Neutron Energy } \\
(\mathbf{M e V})\end{array}$ & $\begin{array}{c}\Sigma_{\mathrm{T}} \\
\left(\mathbf{c m}^{-\mathbf{1}}\right)\end{array}$ & $\begin{array}{c}\text { Mean-free-path } \\
(\mathbf{c m})\end{array}$ & $\begin{array}{c}\text { Equivalent } \\
\text { PWR Pins } \\
(\mathbf{n o .})\end{array}$ \\
\hline $1 \mathrm{eV}-0.2 \mathrm{MeV}$ & 0.4175 & 2.395 & 3.29 \\
10.0 & 0.2087 & 4.790 & 6.59 \\
20.0 & 0.2204 & 4.538 & 6.24 \\
\hline
\end{tabular}


From Table 3 it is clear that fast neutrons with an energy in the $10-$ to $20-\mathrm{MeV}$ range have double the mean-free-path in $\mathrm{UO}_{2}$ that lower-energy epithermal neutrons have in the $1.0-\mathrm{eV}$ to $0.2-\mathrm{MeV}$ range. Hence, the fast neutrons can be expected to have greater penetration capability (6.59 pins per mfp) and signal differentiation for 1-12 pins per row. Note: neutrons of energy between 3-10 MeV should also exhibit the same penetration behavior as the mono-energetic $10 \mathrm{MeV}$ neutrons because their total cross sections are very similar over this energy range.

Figure 47 shows the MCNP-calculated transmission total flux (neutrons $/ \mathrm{cm}^{2} /$ source-neutron) for monoenergetic neutron beams of $29 \mathrm{eV}, 10 \mathrm{MeV}$, and $20 \mathrm{MeV}$ through 0-12 PWR fuel pins with $30 \mathrm{GWD} / \mathrm{MTU}$ spent fuel. The calculated flux is the total energy-integrated transmission flux. The MCNP models utilized air-filled flight tubes and, hence, the three values for the three neutron energy cases for the zero "number of in-line fuel pins" do not match up exactly due to energy-dependent elastic scattering.

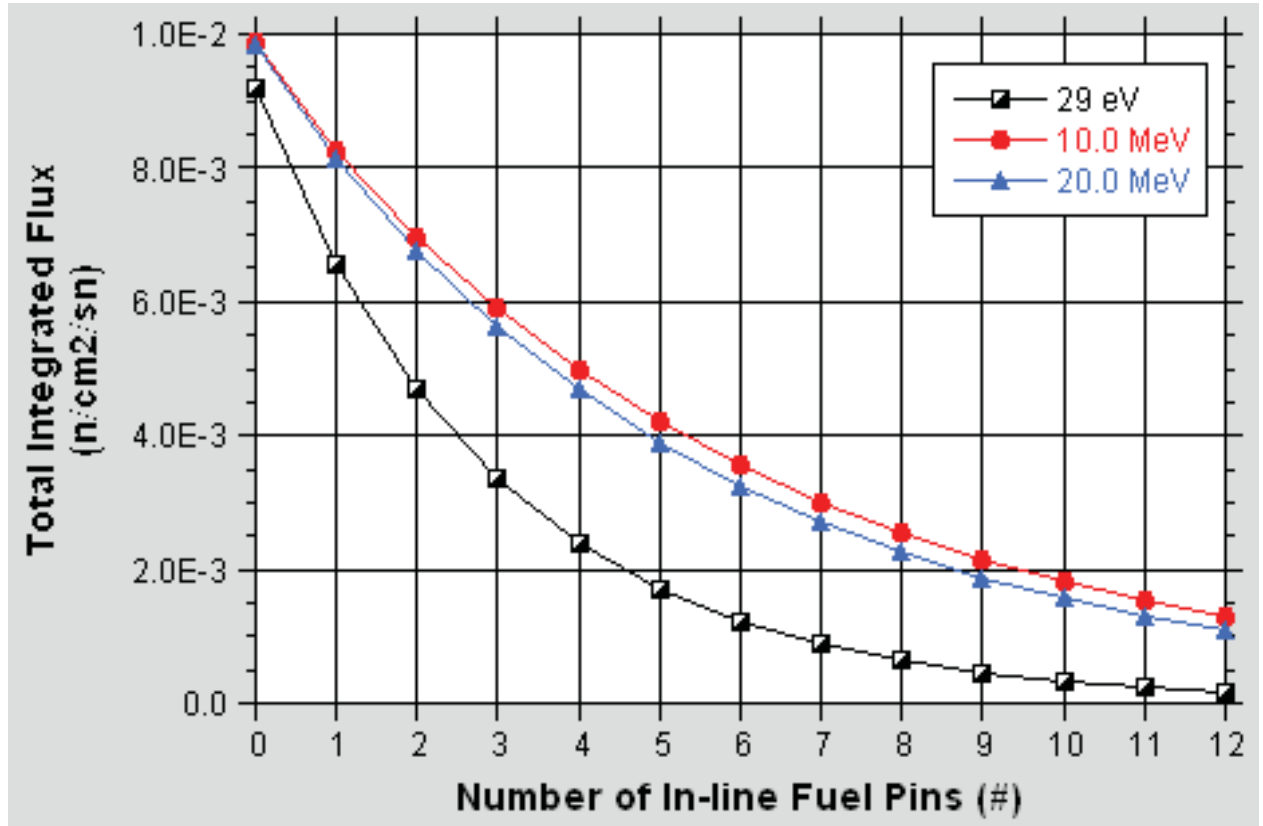

Figure 47 Total integrated transmission flux for mono-energetic neutron beams incident on rows of $\mathrm{PWR} \mathrm{UO}_{2}$ spent fuel pins.

The high-energy 10 - and $20-\mathrm{MeV}$ transmission fluxes decrease more slowly than the $29-\mathrm{eV}$ flux; the $29-\mathrm{eV}$ flux case is in a resonance-free region in the 0 to $40-\mathrm{eV}$ energy range and would be typical of other measured potential scattering fluxes between the resonance structures of spent $\mathrm{UO}_{2}$ fuel. The $10-$ and $20-\mathrm{MeV}$ fluxes (transmission signals) decrease slower and more linearly than the $29-\mathrm{eV}$ curve, such that the flux decrement between fuel pins, particularly in the important 6-12 pins-in-a-line range, is more uniform and hence more readily differentiable than the $29-\mathrm{eV}$ case.

Since the NRTA system is fully capable of producing high-energy photoneutrons $(2-10 \mathrm{MeV})$, or higher energy neutrons depending on the accelerator's maximum electron energy. These fast neutrons could conceivably be used to achieve better penetration through reduced beam out-scatter for the specific purpose of interrogating 1-12 in-line 
PWR spent fuel pins (sufficient to directly scan each pin in the assembly using multiple views). Also, assuming equivalent accelerator operating conditions (beam current and energy), the neutron flux in the "fast mode" would be higher than in the "normal mode," and the measured flux (counts) would be based on the time-integrated counts and not the time-bin parsed counts in the "normal mode." Hence, the "fast mode" signal should be considerably stronger, which would translate into shorter count times.

\section{CONCEPTUAL SYSTEM DESIGN}

This section identifies system variables, compiles results from the conceptual or feasibility studies above, and provides a quantitative estimate or qualitative description for each variable. The intent is to begin to develop preliminary requirements for an NRTA conceptual system design. Estimates are based on both the conceptual design studies above and published results. For this analysis an electron accelerator has been considered as the basis of the neutron source. Generally speaking, electron accelerator systems are less complicated and more straightforward to use for broad-energy, highintensity neutrons sources. Future NRTA studies will be needed to evaluate the potential benefits of using charged-particle accelerators as the basis of an NRTA neutron source for spent-fuel assay measurements.

\subsection{Maximum Electron Energy}

An electron accelerator operating in a pulsed mode of operation is one tool capable of producing neutrons for the time-of-flight NRTA technique. The accelerator will eventually be sized according to the maximum-electron-energy and beam-current requirements. In order to maximize the beam current for a given power the maximum electron energy should be as low as possible. For the NRTA technique applied to the NGSI plutonium assay challenge preliminary analysis suggests that a maximum electron energy of $10-12 \mathrm{MeV}$ should be sufficient. The $10-12 \mathrm{MeV}$ energy range is relatively low for time-of-flight measurements; most electron accelerators used for cross-section measurement run in the range of $100 \mathrm{MeV}$. The low-energy for the NRTA system is based on the $2.22 \mathrm{MeV}$ photoneutron threshold for deuterium (heavy hydrogen) and/or the $1.67 \mathrm{MeV}$ photoneutron threshold for beryllium and recognition that the system does not need to produce high-intensity, higher-energy, time-resolved neutron radiation greater than $40 \mathrm{eV}$. Bremsstrahlung photon continuums with maximum electron energies of 10$12 \mathrm{MeV}$ should be reasonable, although going up to $15 \mathrm{MeV}$ would maximize the $(\gamma, \mathrm{n})$ cross section for beryllium in the giant dipole resonance region. Also, operating in the 10 $\mathrm{MeV}$ range an NRTA accelerator will produce relatively low energy photoneutrons that will help reduce the needed thickness of the neutron moderator, reducing the loss of neutrons in this system component.

A variable-energy electron accelerator would provide additional versatility for the NRTA technique. Variable-energy electrons over the 4-20 MeV range could provide higher- or lower-energy neutrons and/or photons for alternative radiation probes, in addition to optimizing the NRTA technique to maximize the source neutron output. Of key importance here is the fact that the lower the maximum electron energy the higher the beam current may be for a constant power-on-target rating. NRTA will require an intense neutron source to reduce count times. 


\subsection{Timing Signal}

The timing signal is typically initiated by a photomultiplier tube located in the vicinity of the electron converter. When the electrons strike the converter, this represents $\mathrm{t}=0$ for a pulse sequence; this is recorded in the data acquisition system for correlating the neutron detector events with each accelerator pulse. Conversion of the electrons to bremsstrahlung photons, conversion of the photons into photoneutrons, and fast photoneutron thermalization occur relatively quickly, such that the transit time of the thermal and epithermal neutrons down the flight tube to the detectors takes the vast majority of the total flight time.

\subsection{Neutron Resonance Energy}

Based on the results of the conceptual studies above, the resonance neutron energy range most pertinent to the NRTA technique appears to be $0.1-20 \mathrm{eV}$. Published literature suggests a range up to $40 \mathrm{eV}$. However, no additional resonance data appears to be gained in the $20-40 \mathrm{eV}$ range. Plus, the $0.1-20 \mathrm{eV}$ range can be achieved with high resolution and short flight-tube lengths. The region above $40 \mathrm{eV}$ was not explored in detail but would require longer flight-tube lengths; also, resonances in these energies are typically smaller and spaced closer together.

\subsection{Flight-Tube Length}

The flight-tube length will be based on several interconnected design variables involving the accelerator, the transmission neutron spectrum and intensity, and the detectors. These variables include:

1. Resonance neutron energy range,

2. Accelerator pulse rate,

3. Accelerator pulse width, and

4. Detector time-resolution.

From the results of the conceptual studies above, the resonance neutron energy range is proposed to be $0.1-20 \mathrm{eV}$. The accelerator pulse rate, or repetition rate, should be as high as possible to maximize count rates, yet not so high that fast neutrons from one cycle overrun the slow neutrons in the previous cycle. A pulse rate in the range of 700$750 \mathrm{~Hz}$ would have cycle lengths of 1,330-1,429 microseconds. The accelerator pulse width can range from 5-500 ns, or 0.005-0.5 microseconds. It needs to be short relative to the cycle length and neutron transit times (approximating a delta function). The pulse width will also be a consideration in the accelerator design, taking into account methods to maximize the photon output per pulse. For the desired $0.1-20 \mathrm{eV}$ resonant energy range for

transmission neutrons, 
Table 4 gives neutron flight times (microseconds) as a function of neutron energy $(\mathrm{eV})$, neutron velocity $(\mathrm{cm} / \mathrm{s})$, and flight-tube length (meters). For completeness, the data in this table are for $0.1-40 \mathrm{eV}$. Table 5 gives the accelerator pulse rates and corresponding cycle lengths. 
Table 4 Neutron flight times for different flight tube lengths and neutron energies.

\begin{tabular}{|c|c|c|c|c|c|c|c|c|}
\hline \multirow{3}{*}{$\begin{array}{l}\text { Neutron } \\
\text { Energy } \\
(\mathrm{eV})\end{array}$} & \multirow{3}{*}{$\begin{array}{c}\text { Neutron } \\
\text { Velocity } \\
(\mathrm{cm} / \mathrm{s})\end{array}$} & \multirow{3}{*}{$\begin{array}{c}\text { Transit Time } \\
\text { Over } 0.2 \mathrm{~cm} \\
(\mu \mathrm{s})\end{array}$} & \multirow{2}{*}{\multicolumn{6}{|c|}{$\begin{array}{l}\text { Neutron flight times }(\mu \mathbf{s}) \\
\text { Flight Tube Length (meters) }\end{array}$}} \\
\hline & & & & & & & & \\
\hline & & & 1.0 & 2.0 & 4.0 & 6.0 & 8.0 & 10.0 \\
\hline 0.10 & $4.37 \mathrm{E}+05$ & 0.457 & 229 & 457 & 915 & 1,372 & 1,829 & 2,287 \\
\hline 0.20 & $6.18 \mathrm{E}+05$ & 0.323 & 162 & 323 & 647 & 970 & 1,293 & 1,617 \\
\hline 0.30 & $7.58 \mathrm{E}+05$ & 0.264 & 132 & 264 & 528 & 792 & 1,056 & 1,320 \\
\hline 0.40 & $8.75 \mathrm{E}+05$ & 0.229 & 114 & 229 & 457 & 686 & 915 & 1,143 \\
\hline 0.50 & $9.78 \mathrm{E}+05$ & 0.205 & 102 & 205 & 409 & 614 & 818 & 1,023 \\
\hline 0.60 & $1.07 \mathrm{E}+06$ & 0.187 & 93 & 187 & 373 & 560 & 747 & 933 \\
\hline 0.70 & $1.16 \mathrm{E}+06$ & 0.173 & 86 & 173 & 346 & 519 & 691 & 864 \\
\hline 0.80 & $1.24 \mathrm{E}+06$ & 0.162 & 81 & 162 & 323 & 485 & 647 & 808 \\
\hline 0.90 & $1.31 \mathrm{E}+06$ & 0.152 & 76 & 152 & 305 & 457 & 610 & 762 \\
\hline 1.00 & $1.38 \mathrm{E}+06$ & 0.145 & 72 & 145 & 289 & 434 & 578 & 723 \\
\hline 2.00 & $1.96 \mathrm{E}+06$ & 0.102 & 51 & 102 & 205 & 307 & 409 & 511 \\
\hline 3.00 & $2.40 \mathrm{E}+06$ & 0.083 & 42 & 83 & 167 & 250 & 334 & 417 \\
\hline 4.00 & $2.77 \mathrm{E}+06$ & 0.072 & 36 & 72 & 145 & 217 & 289 & 362 \\
\hline 5.00 & $3.09 \mathrm{E}+06$ & 0.065 & 32 & 65 & 129 & 194 & 259 & 323 \\
\hline 6.00 & $3.39 \mathrm{E}+06$ & 0.059 & 30 & 59 & 118 & 177 & 236 & 295 \\
\hline 7.00 & $3.66 \mathrm{E}+06$ & 0.055 & 27 & 55 & 109 & 164 & 219 & 273 \\
\hline 8.00 & $3.91 \mathrm{E}+06$ & 0.051 & 26 & 51 & 102 & 153 & 205 & 256 \\
\hline 9.00 & $4.15 \mathrm{E}+06$ & 0.048 & 24 & 48 & 96 & 145 & 193 & 241 \\
\hline 10.00 & $4.37 \mathrm{E}+06$ & 0.046 & 23 & 46 & 91 & 137 & 183 & 229 \\
\hline 11.00 & $4.59 \mathrm{E}+06$ & 0.044 & 22 & 44 & 87 & 131 & 174 & 218 \\
\hline 12.00 & $4.79 \mathrm{E}+06$ & 0.042 & 21 & 42 & 83 & 125 & 167 & 209 \\
\hline 13.00 & $4.99 \mathrm{E}+06$ & 0.040 & 20 & 40 & 80 & 120 & 160 & 201 \\
\hline 14.00 & $5.17 \mathrm{E}+06$ & 0.039 & 19 & 39 & 77 & 116 & 155 & 193 \\
\hline 15.00 & $5.36 \mathrm{E}+06$ & 0.037 & 19 & 37 & 75 & 112 & 149 & 187 \\
\hline 16.00 & $5.53 \mathrm{E}+06$ & 0.036 & 18 & 36 & 72 & 108 & 145 & 181 \\
\hline 17.00 & $5.70 \mathrm{E}+06$ & 0.035 & 18 & 35 & 70 & 105 & 140 & 175 \\
\hline 18.00 & $5.87 \mathrm{E}+06$ & 0.034 & 17 & 34 & 68 & 102 & 136 & 170 \\
\hline 19.00 & $6.03 \mathrm{E}+06$ & 0.033 & 17 & 33 & 66 & 100 & 133 & 166 \\
\hline 20.00 & $6.18 \mathrm{E}+06$ & 0.032 & 16 & 32 & 65 & 97 & 129 & 162 \\
\hline 21.00 & $6.34 \mathrm{E}+06$ & 0.032 & 16 & 32 & 63 & 95 & 126 & 158 \\
\hline 22.00 & $6.49 \mathrm{E}+06$ & 0.031 & 15 & 31 & 62 & 92 & 123 & 154 \\
\hline 23.00 & $6.63 \mathrm{E}+06$ & 0.030 & 15 & 30 & 60 & 90 & 121 & 151 \\
\hline 24.00 & $6.78 \mathrm{E}+06$ & 0.030 & 15 & 30 & 59 & 89 & 118 & 148 \\
\hline 25.00 & $6.92 \mathrm{E}+06$ & 0.029 & 14 & 29 & 58 & 87 & 116 & 145 \\
\hline 26.00 & $7.05 \mathrm{E}+06$ & 0.028 & 14 & 28 & 57 & 85 & 113 & 142 \\
\hline 27.00 & $7.19 \mathrm{E}+06$ & 0.028 & 14 & 28 & 56 & 83 & 111 & 139 \\
\hline 28.00 & $7.32 \mathrm{E}+06$ & 0.027 & 14 & 27 & 55 & 82 & 109 & 137 \\
\hline 29.00 & $7.45 \mathrm{E}+06$ & 0.027 & 13 & 27 & 54 & 81 & 107 & 134 \\
\hline 30.00 & $7.58 \mathrm{E}+06$ & 0.026 & 13 & 26 & 53 & 79 & 106 & 132 \\
\hline 31.00 & $7.70 \mathrm{E}+06$ & 0.026 & 13 & 26 & 52 & 78 & 104 & 130 \\
\hline 32.00 & $7.82 \mathrm{E}+06$ & 0.026 & 13 & 26 & 51 & 77 & 102 & 128 \\
\hline 33.00 & $7.94 \mathrm{E}+06$ & 0.025 & 13 & 25 & 50 & 76 & 101 & 126 \\
\hline 34.00 & $8.06 \mathrm{E}+06$ & 0.025 & 12 & 25 & 50 & 74 & 99 & 124 \\
\hline 35.00 & $8.18 \mathrm{E}+06$ & 0.024 & 12 & 24 & 49 & 73 & 98 & 122 \\
\hline 36.00 & $8.30 \mathrm{E}+06$ & 0.024 & 12 & 24 & 48 & 72 & 96 & 121 \\
\hline 37.00 & $8.41 \mathrm{E}+06$ & 0.024 & 12 & 24 & 48 & 71 & 95 & 119 \\
\hline 38.00 & $8.53 \mathrm{E}+06$ & 0.023 & 12 & 23 & 47 & 70 & 94 & 117 \\
\hline 39.00 & $8.64 \mathrm{E}+06$ & 0.023 & 12 & 23 & 46 & 69 & 93 & 116 \\
\hline 40.00 & $8.75 \mathrm{E}+06$ & 0.023 & 11 & 23 & 46 & 69 & 91 & 114 \\
\hline
\end{tabular}


Table 5 Accelerator pulse rate and corresponding cycle length.

\begin{tabular}{cc}
\hline $\begin{array}{c}\text { Pulse Rate } \\
(\mathbf{H z})\end{array}$ & $\begin{array}{c}\text { Cycle Length } \\
(\boldsymbol{\mu} \mathbf{s})\end{array}$ \\
\hline 100 & 10,000 \\
200 & 5,000 \\
300 & 3,333 \\
400 & 2,500 \\
500 & 2,000 \\
600 & 1,670 \\
700 & 1,429 \\
720 & 1,390 \\
750 & 1,330 \\
800 & 1,250 \\
\hline
\end{tabular}

From the data in Table 4 it is apparent that low-energy (low-velocity) neutrons $(0.1-1.0 \mathrm{eV})$ separate or spread out well as they travel down a fixed-length flight tube and, therefore, are relatively easy to resolve in energy and time. Neutrons of slightly higher energy $(15-20 \mathrm{eV})$ travel faster and do not spread out nearly as much for the same length flight tube and, consequently, are more difficult to resolve. Hence, low-energy neutrons can use shorter flight-tube lengths than higher-energy neutrons. Accelerator systems designed for cross-section measurements in the $\mathrm{keV}$ energy range require flight tube lengths as long as 200-meters. The NRTA technique with its $0.1-20 \mathrm{eV}$ energy neutrons can use much shorter flight-tube with lengths of less than 10 meters.

The NRTA flight-tube length will be determined by balancing the resonant neutron energy range, time resolution of the transmission signal, and count time. Count time will also be impacted by accelerator neutron output. The flight-tube length cannot be too short or too long for the NRTA energy range $(0.1-20 \mathrm{eV})$. If it is too short, the higher energy neutrons $(20 \mathrm{eV})$ will be difficult to resolve without very short time channel widths. If the flight tube is too long, the low-energy neutrons $(0.1 \mathrm{eV})$ could be overrun by very fast $(\mathrm{MeV})$ neutrons from the next cycle unless, of course, the repetition rate is reduced, which in turn affects the accelerator neutron output and count time.

The low end of the desired resonant neutron energy range $(0.1 \mathrm{eV})$ is needed in order to resolve the $0.3 \mathrm{eV}{ }^{239} \mathrm{Pu}$ resonance. A $700 \mathrm{~Hz}$ pulse rate, or $1,429 \mu$ s cycle length, with a 6-meter flight tube would be sufficient to allow these very low-energy neutrons (with a flight time of $1,372 \mu \mathrm{s}$ ) to make it to the detectors before the next pulse begins. The higher-energy neutrons $(20 \mathrm{eV})$ can then still be resolved with a detector having $0.5-\mu$ s time resolution. A 4-meter flight tube would also work; however reducing the flight tube length further to only 2-meters will start to significantly impact the resolution of the higher-energy neutrons unless the time detector's time resolution can be improved to 0.1-0.25 microseconds.

In Table 4 the time difference ( $\mu$ s) between flight times for adjacent neutron energies will give the signal resolution time ( $\mu \mathrm{s})$, or the corresponding number of time bins, or separate count channels, between adjacent neutron energies using $1-\mu$ s channel widths. Better detector time-resolution is possible with finer channel widths at the expense of fewer counts per channel for a given count period. 
There is some impetus in the design of the NRTA system to use as short a flighttube length as possible. A shorter flight-tube length would reduce the NRTA system footprint and reduce the time required to pump-down the tube to vacuum conditions. Both conditions would aid in the goal of a small mobile NRTA system design too. A thermal neutron filter $(\mathrm{Cd}, \mathrm{Gd})$ may be required to absorb very slow neutrons $(<0.1 \mathrm{eV})$ in order to maximize the pulse rate and reduce the cycle length.

\subsection{Flight-Tube Design}

Based on the feasibility study involving vacuum versus air in the flight tube it was determined that the flight tube would need to be in vacuum but could be divided into two pieces, a fore and aft section. There would be a section linking the accelerator and fore section and a section linking the aft section and the detector array, this would be a split flight-tube design with the fuel in the middle. A split flight tube avoids one long tube, allows for the central chamber containing the fuel assembly to be air-filled and aircooled, provides flexibility to accommodate targets, converters, and filters in the fore section, and allows better designs for shielding and detectors in the aft section. The two evacuated tube sections would be separate units. The accelerator system would be a third unit that would mate with the fore-tube section; fore and aft sections mating with the central chamber. Subdivision of the NRTA system into reasonable size and weight components may allow some degree of system mobility.

The flight-tube cross section, size and geometry have not been determined. The cross section geometry and size will be dependent on the detector geometry; a rectangular cross section might be the most practical.

\subsection{Detectors}

Neutron detectors for the NRTA system have not been addressed to date. However, a preliminary assessment and a few ideas are offered for future consideration. Helium-3 and lithiated-glass scintillators are potential detectors for the NRTA system and are routinely used in cross section measurements. Helium-3 detectors based on the ${ }^{3} \mathrm{He}(\mathrm{n}, \mathrm{p}){ }^{3} \mathrm{H}$ reaction with corresponding large cross section (approximately 1000 barns at $1.0 \mathrm{eV}$ ) and exothermic Q-value of $764 \mathrm{keV}$ are attractive for slow-neutron detection. Helium-3 detectors can exhibit some sensitivity to gamma radiation in the form of pulse pileup and potential interference with the neutron pulses.[5] The intense spent-fuel gamma field will need to be shielded appropriately.

Lithium-6 glass scintillator detectors may also prove useful as high-rate count detectors, in particular, a Li-glass scintillator (NE912) enriched to $95 \%{ }^{6} \mathrm{Li} .[3,8]$ As a slow-neutron detector the ${ }^{6} \mathrm{Li}(\mathrm{n}, \alpha) \mathrm{t}$ reaction has a large exothermic Q-value of $4.78 \mathrm{MeV}$, strong $1 / \mathrm{v}$ thermal neutron cross section (approximately 200 barns at $1.0 \mathrm{eV}$ ), 7.7\% lithium content (NE912), and fast response time. In addition, natural- or depleted-lithium scintillator detectors can be used in conjunction with the enriched detectors to estimate the gamma contribution in a neutron-gamma flux.[5]

Glass scintillators can also be fabricated in a variety of geometric forms and sizes, which include large area sections and small-diameter optical fibers. The small-diameter fibers are a very attractive feature with diameters as small $100 \mu \mathrm{m}$ or less. At these small diameters the fibers would minimize gamma energy deposition and, lumped together into a fiber bundle (fiber diameter $<1-2 \mathrm{~mm}$ ), could spatially resolve neutron transmission 
fluxes across the diameter of a fuel pin. This latter feature is very attractive and could possibly allow for 12-pin assays (12 in-line pins in a fuel assembly), thus permitting NRTA to assay an entire PWR assembly.

\subsection{Shielding}

No shielding-design studies have been performed to date. Minimizing the number of background neutrons striking the detectors can be accomplished by maximizing the distance between the assembly and detectors and/or installing neutron and gamma shielding between the assembly and the detectors. From the conceptual study involving spent-fuel neutron emission it was shown that background neutron counts would be negligible if neutron shielding is placed in front of or around the flight tube. Similar arguments can be made for gamma shielding.

\subsection{Alternative Operating Modes}

The standard mode of operation for the NRTA technique is with low-energy neutrons $(0.1-20 \mathrm{eV})$. The NRTA system however also has the capability to produce other interrogating radiations. For example, removal of the low-Z neutron moderating filter can produce a beam of fast or higher-energy neutrons in the 1-20 MeV range. Use of these fast neutrons is discussed section 4.14 "Missing Fuel Pins." In addition, highenergy photon beams can be produced by removing the photon-to-neutron converter. Operating the accelerator with maximum electron energy in the 10-20 MeV would produce a distribution of bremsstrahlung photons in this "giant dipole resonance" energy range. Directed at a fuel assembly, the photon beam would induce photo-neutrons and photo-fissions in the spent fuel materials.

\subsection{Design Parameter Summary}

Table 6 contains a list of NRTA system design variables and preliminary variable estimates. The list is not comprehensive. It is intended to be a dynamic table where new variables can be added and estimates updated. As the INL NRTA project continues to analyze various aspects and components of the NRTA technique and system, the summary table below should evolve into a viable conceptual design. 
Table 6 Initial NRTA System Parameters

\begin{tabular}{|c|c|c|}
\hline & Design Parameter & Parameter Values/Ranges \\
\hline \multirow{6}{*}{ Accelerator: } & Beam power & TBD \\
\hline & Beam current & TBD \\
\hline & Maximum electron energy, $\mathrm{MeV}$ & $10-12$ \\
\hline & Electron pulse width, ns & $5-500$ \\
\hline & Repetition rate, $\mathrm{Hz}$ & $700-800$ \\
\hline & Cycle length, $\mu \mathrm{s}$ & $1,429-1,250$ \\
\hline \multirow{7}{*}{$\begin{array}{l}\text { Targets/ } \\
\text { Converters/ } \\
\text { Filters: }\end{array}$} & Electron converter material & Tungsten \\
\hline & Electron converter thickness, $\mathrm{cm}$ & 0.3 \\
\hline & Photon converter material & ${ }^{2} \mathrm{H}$ or ${ }^{9} \mathrm{Be}$ \\
\hline & Photon converter thickness, $\mathrm{cm}$ & TBD \\
\hline & Neutron moderator material & Polyethylene, $\mathrm{H}_{2} \mathrm{O}, \mathrm{D}_{2} \mathrm{O}$ \\
\hline & Neutron moderator thickness, $\mathrm{cm}$ & TBD \\
\hline & Thermal neutron filter material & $\mathrm{Cd}, \mathrm{Gd}$ \\
\hline \multirow{3}{*}{$\begin{array}{l}\text { Transmission } \\
\text { Neutrons: }\end{array}$} & Resonance energy range, eV & $0.1-20$ \\
\hline & Flight time (4-m tube), $\mu$ s & 915 \\
\hline & Flight time (6-m tube), $\mu \mathrm{s}$ & 1,372 \\
\hline \multirow{4}{*}{$\begin{array}{l}\text { Flight } \\
\text { Tube: }\end{array}$} & Diameter & TBD \\
\hline & Total Length, m & $4-6$ \\
\hline & Wall material & Aluminum \\
\hline & Fill material & Vacuum \\
\hline \multirow{3}{*}{$\begin{array}{l}\text { Assembly } \\
\text { Chamber: }\end{array}$} & Fill material & Air (air-cooling) \\
\hline & Wall material & Aluminum \\
\hline & Wall thickness & TBD \\
\hline \multirow{2}{*}{ Shielding: } & Neutron & Borated polyethylene \\
\hline & Photon & TBD \\
\hline \multirow{2}{*}{$\begin{array}{l}\text { Neutron } \\
\text { Detectors: }\end{array}$} & Type & $\mathrm{He}^{3}$ or $\mathrm{Li}^{6}$-glass scintillator \\
\hline & Minimum time resolution, $\mu \mathrm{s}$ & 0.1 \\
\hline
\end{tabular}

\section{FUTURE WORK}

The following tasks are planned for fiscal year 2011.

1. Complete an NRTA Preliminary Studies Report.

2. Prepare documentation and presentation materials for the NGSI 2011 technical review panel for the Plutonium Assay Challenge.

3. Address issues raised during the 2011 technical review panel.

4. Carry out additional integration research needed to proceed with evaluating the NRTA concept for spent fuel assay.

5. Develop high-fidelity NRTA simulations building upon the work in this report.

- Creation of a realistic scenario model including accelerator and detector specific properties, and including fuel background radiation signatures. 
- Quantify the simulated response of the NRTA technique for the analysis of the LANL spent fuel assemblies.

6. Submit a draft report by the end of May 2011 that summarizes the entire project.

Some prioritized research questions and topic areas that still require investigation regarding the NRTA concept include the following.

1. Develop numerical benchmarks and preliminary analytical tools to calculate isotopic assays from calculated neutron transmission spectra.

2. What measurement precision can an NRTA system be reasonably expected to achieve?

3. How well can NRTA address the three LANL-defined PWR fuel-pin diversion scenarios?

4. Correct or develop new MCNP deuterium photonuclear cross sections; errors currently in the deuterium cross sections lead MCNP to calculate incorrect photoneutron energies.

5. What is the optimal accelerator system to use for an NRTA system?

6. How should neutron production be optimized for an NRTA system? Using the electron accelerator concept as a baseline, this will require converter design and parametric studies to understand how to increase the neutron output from the bremsstrahlung photon-to-neutron converter optimally for NRTA.

7. What is the optimal moderator-filter design for NRTA? This will require studies to minimize the filter thickness and achieve maximum thermal and epithermal neutrons in the $0-20 \mathrm{eV}$ neutron energy range for the slowing-down spectrum.

8. What is the best solution for neutron detection for an NRTA system?

\section{CONCLUSIONS}

A preliminary assessment of the nondestructive assay technique known as neutron resonance transmission analysis (NRTA) has been performed by Idaho National Laboratory. The assessment is not complete but preliminary results indicate the NRTA to be a powerful assay technique for spent fuel. It can identify four plutonium isotopes $\left({ }^{239,240,241,242} \mathrm{Pu}\right)$, three uranium isotopes $\left({ }^{235,236,238} \mathrm{U}\right)$, and six resonant fission products. This technqiue can determine the areal density or mass of these isotopes in single- or multiple-pin integral transmission scans. Published literature suggests assay an accuracy of $1-4 \%$ uncertainty for these isotopes.[30]

The NRTA technique has specifically been assessed for a pressurized water reactor spent fuel assembly with a $17 \times 17$ pin array. The calculated results suggest sufficient transmission strength and signal differentiability to assay up to 8-pin rows. For an 8-pin maximum, $64 \%$ of the fuel pins in an assembly can be part of an integral transmission assay measurement. Collimated neutron beams or neutron detector designs that can spatially resolve a fuel-pin diameter have the potential to assay up to 12-pin 
rows. For a 12-pin maximum, $100 \%$ of the fuel pins in an assembly can be part of one or more integral transmission assay measurements.

The NRTA technique requires an intense, pulsed neutron source in order to achieve sufficient neutron transmission through the assembly for reasonable count times and to allow the time-of-flight method employed here to measure neutron energy/velocity. The most efficient system with both of these capabilities is a linear electron accelerator, although other accelerator types may also be applicable. Part of the fiscal year 2011 NRTA studies will focus on accelerator design to maximize the electronbeam current, converter design to maximize photoneutron output, and moderator-filter design to minimize neutron loss, with the combined goal of maximizing neutron-beam intensity.

A conceptual NRTA system design has been presented and will continue to evolve with new feasibility and system performance studies. The conceptual system design is far from complete, and the system variables are complexly interconnected with strong dependencies on still unknown and important variables. The design offers numerous optimization areas with flexibility to optimize the system for specific goals. For example, if the main goal was assay accuracy, an NRTA system with a relatively large footprint could be designed. If a transportable system capability was a design goal, a smaller footprint system consisting of several units (table-top-sized accelerator, two separate evacuated flight-tube sections, and detector/electronics) could be assembled around a chamber containing the spent fuel assembly. 


\section{REFERENCES}

1 Tobin, S. J., et al., "Next Generation Safeguards Initiative Research to Determine the $\mathrm{Pu}$ Mass in Spent Fuel Assemblies: Purpose, Approach, Constraints, Implementation, and Calibration," Nucl. Inst. Meth. Phys. Res. A, doi:10.1016/j.nima.2010.09.064 (in press).

2 Schrack, R.A., et al. "Resonance Neutron Radiography using an Electron Linac," IEEE Trans. Nucl. Sci. 28 (1981) 1640-1643.

3 Bowman, C.D., et al., "Neutron Resonance Transmission Analysis of Reactor Spent Fuel Assemblies," Neutron Radiography, Barton, J. P. and von der Hardt, P., eds., ECSC, EEC, EAEC, Brussels, Belgium and Luxembourg (1983) 503-511.

4 Behrens, J. W., Johnson, R. G., and Schrack, R. A., "Neutron Resonance Transmission Analysis of Reactor Fuel Samples,” Nucl. Tech. 67 (1984) 162-168.

5 Knoll, G. F., Radiation Detection and Measurement, $3^{\text {rd }}$ edition, John Wiley and Sons, Inc., Hoboken, N. J. (2000) Chs. 8, 14, and 15.

6 Gavron, A., Smith, L. E., and Ressler, J. J., "Analysis of Spent Fuel Assemblies using a Lead Slowing Down Spectrometer," Nucl. Inst. Meth. Phys. Res. A 602 (2009) 581-587.

7 Priesmeyer, H. G. and Harz, U., "Isotopic Assay in Irradiated Fuel by Neutron Resonance Analysis," Atomkernenergie (ATKE) 25 (1975) 109-113.

8 Noguere, G., et al., "Non-destructive Analysis of Materials by Neutron Resonance Transmission,” Nucl. Inst. Meth. Phys. Res. A 575 (2007) 476-488.

9 Schrack, R. A., "Uranium-235 Measurement in Waste Material by Resonance Neutron Radiography,” Nucl. Tech. 67 (1984) 326-332.

10 Schrack, R. A., "New Applications of Resonance Neutron Radiography," International Conference on Nuclear Data for Basic and Applied Science, Santa Fe, N.M., May 13-17 (1985).

11 Johnson, R. G. and Schrack, R. A., "Nondestructive Evaluation of M732 Proximity Fuzes," Report NBSIR 85-3259, National Bureau of Standards, Gaithersburg, Md., November (1985).

12 Behrens, J. W., Schrack, R. A., and Bowman, C. D., "Nondestructive Examination of a Defective Silver Braze using Resonance-Neutron Radiography," Nucl. Tech. 51 (1980) 178-182.

13 Schrack, R. A., "U-235 Measurement by Resonance Neutron Radiography," Report RADP/B119 NBS, Final report to the U.S. Nuclear Regulatory Commission, National Bureau of Standards, Gaithersburg, Md., June 30 (1983).

14 Bowman, C. D., "Efficient Neutron Production Using Low-Energy Electron Beams," Proc. Int. Conf. on Nuclear Cross Sections for Tech., Univ. of Tennessee, Knoxville, Tenn., Oct. 22-26 (1980) 531. 
15 Jones, J. L., et al., "Pulsed Photoneutron Interrogation: The GNT Demonstration System," Report WINCO-1225, Idaho National Environmental and Engineering Laboratory, Idaho Falls, Id. (1994).

16 See, for example, the Lansar Neutron Generator from Accsys Technologies, Inc. (www.accsys.com/products/lansar.html).

17 The Geel Electron Linear Accelerator (GELINA) neutron time-of-flight facility: irmm.jrc.ec.europa.eu/about_IRMM/laboratories/Pages/gelina_neutron_time_of_fli ght_facility.aspx

18 The Oak Ridge Electron Linear Accelerator (ORELA) pulsed neutron source: www.phy.ornl.gov/orela/orela.html/

19 "MCNP-A General Monte Carlo N-Particle Transport Code, Version 5," X-5 Monte Carlo Team, Report LA-UR-03-1987, Los Alamos National Laboratory, Los Alamos, N.M. (2003).

20 "MCNPX - A General Purpose Monte Carlo Radiation Transport Code, Version 2.5.0," MCNPX User's Manual, LA-UR-05-0369, Los Alamos National Laboratory, Los Alamos, N.M. (2005).

21 Private communications, Steve Tobin, Los Alamos National Laboratory (2010).

22 Chadwick, M. B., et al., "ENDF/B-VII.0: Next Generation Evaluated Nuclear Data Library for Nuclear Science and Technology," Nucl. Data Sheets 107 (2006) 29313060 .

23 Fensin, M. L., et al., "A Monte Carlo Linked Depletion Spent Fuel Library for Assessing Varied Nondestructive Assay Techniques for Nuclear Safeguards," Report LA-UR-09-01188, Los Alamos National Laboratory, Los Alamos, N.M. (2009).

24 Ludwig, S. B. and Croft, A. G., "ORIGEN2.2-Isotope Generation and Depletion code - Matrix Exponential Method," Report CCC-371, Oak Ridge National Laboratory, Oak Ridge, Tenn. (2002).

25 Sher, R. and Untermyer, S., The Detection of Fissionable Materials by Nondestructive Means, American Nuclear Society, La Grange, Ill. (1980).

26 Table of Isotopes, $7^{\text {th }}$ edition, Lederer, C. M. and Shirley, V. S., eds., WileyInterscience, New York, N.Y. (1978).

27 Ensslin, N., "The Origin of Neutron Radiation," presented in "Passive Nondestructive Assay of Nuclear Materials", Reilly, D., et al., eds., Report NUREG/CR-5550, U.S. Nuclear Regulatory Commission, Washington, D. C. (1991) 337-356.

28 Richard, J. G., et al., "Burnup-Dependent Neutron Source Term for Gross Neutron Detection Technique," Report LA-UR-10-00075, Los Alamos National Laboratory, Los Alamos, N.M. (2010) and Richard, J. G., et al., "Characterization of the Neutron Source Term and Multiplicity of a Spent Fuel Assembly in Support of 
NDA Safeguards of Spent Nuclear Fuel," Los Alamos National Laboratory, Los Alamos, N.M., not published.

29 Richard, J. G., et al., "Analysis of the Neutron Source Term of a Spent Fuel Assembly," Report LA-UR-10-05384, Los Alamos National Laboratory, Los Alamos, N.M. (2010).

30 Schrack, R.A., "NBS Work on Neutron Resonance Radiography," presented in "Neutron Resonance Radiography," Lynn, J. E. and Hyer, D. K., eds., Report LA11393-C, Los Alamos National Laboratory, Los Alamos, N.M. (1987) 100-115 